\title{
A Complete Leading-Order Renormalization-Scheme-Consistent Calculation of Structure Functions.
}

\author{
Robert S. Thorne \\ Rutherford Appleton Laboratory, \\ Chilton, Didcot, Oxon., OX11 0QX, U.K.
}

\begin{abstract}
We present consistently ordered calculations of the structure functions $\mathcal{F}_{2}\left(x, Q^{2}\right)$ and $\mathcal{F}_{L}\left(x, Q^{2}\right)$, in different expansion schemes. After discussing the standard expansion in powers of $\alpha_{s}\left(Q^{2}\right)$ we consider a leading-order expansion in $\ln (1 / x)$ and finally an expansion which is leading order in both $\ln (1 / x)$ and $\alpha_{s}\left(Q^{2}\right)$, and which is the only really correct expansion scheme. Ordering the calculation in a renormalization-scheme-consistent manner, there is no factorization scheme dependence, and the calculational method naturally includes to the "physical anomalous dimensions" of Catani. However, it imposes stronger constraints than just the use of these effective anomalous dimensions. A relationship between the small $-x$ forms of the inputs $\mathcal{F}_{2}\left(x, Q_{I}^{2}\right)$ and $\mathcal{F}_{L}\left(x, Q_{I}^{2}\right)$ is predicted. Analysis of a wide range of data for $\mathcal{F}_{2}\left(x, Q^{2}\right)$ is performed, and a very good global fit obtained, particularly for data at small $x$. The fit allows a prediction for $\mathcal{F}_{L}\left(x, Q^{2}\right)$ to be produced, which is smaller than those produced by the usual NLO-in- $\alpha_{s}\left(Q^{2}\right)$ fits to $\mathcal{F}_{2}\left(x, Q^{2}\right)$ and different in shape.
\end{abstract}

July 1997 


\section{Introduction.}

The recent measurements of $\mathcal{F}_{2}\left(x, Q^{2}\right)$ at HERA have provided data on a structure function at far lower values of $x$ than any previous experiments, and show that there is a marked rise in $\mathcal{F}_{2}\left(x, Q^{2}\right)$ at very small $x$ down to rather low values of $Q^{2}$ [1][2]. Indeed, the rise persists for values of $Q^{2}$ as low as $1.5 \mathrm{GeV}^{2}$.

The qualitative result of a steep rise at small $x$ was in conflict with standard methods used to fit the data were based on the solution of the Altarelli-Parisi evolution equation [3] at the twoloop level, using nonperturbative, flat (the Donnachie-Landshoff pomeron used to soft physics has behaviour $x^{-0.08}$ [4], and we will take steep to mean any powerlike behaviour steeper than this) input parton distributions at starting scales of $Q_{I}^{2} \sim 4 \mathrm{GeV}^{2}$ (e.g. [5]). This procedure results in an effectively steep behaviour at small $x$ [6], but only after a long evolution length, and therefore at values of $Q^{2} \gg 4 \mathrm{GeV}^{2}$. Thus, the data led to optimism amongst those advocating the use of the BFKL equation [7], which provides the unintegrated gluon Green's function which includes the leading power of $\ln (1 / x)$ for any power of (fixed) $\alpha_{s}$. It was solved analytically in the asymptotic limit $x \rightarrow 0$, or numerically for finite $x$, and predicted a powerlike behaviour of $x^{-1-\lambda}$ for the gluon distribution function, where $\lambda=4 \ln 2 \bar{\alpha}_{s}$ and $\bar{\alpha}_{s}=(3 / \pi) \alpha_{s}$, i.e. $\lambda \sim 0.5$ if $\alpha_{s} \sim 0.2$. This was assumed to lead to $\mathcal{F}_{2}\left(x, Q^{2}\right)$ behaving like $x^{-\lambda}$ and was in rough qualitative agreement with the data, even if $\lambda$ was somewhat high. It could also be seen as some justification for choosing powerlike inputs (with $\lambda \sim 0.2-0.3$ ) for the parton distributions (e.g. [8]), which could then enable a good fit to the data using the Altarelli-Parisi equation.

However, it was also convincingly demonstrated that it was possible to generate the observed steep behaviour by being a little less conservative concerning the region in which perturbative evolution could be applied. Glück, Reya and Vogt had in fact predicted a sharp rise in $F_{2}\left(x, Q^{2}\right)$ at small $x$, even for $Q^{2} \sim 1 \mathrm{GeV}^{2}$, by using two-loop evolution from roughly valence-like parton distributions at a starting scale of $Q_{I}^{2}=0.34 \mathrm{GeV}^{2}[9]$. Starting at a higher scale, $Q_{I}^{2}=1 \mathrm{GeV}^{2}$, Ball and Forte were able to fit the small- $x$ data using their double asymptotic scaling (DAS) formula [10], which is a simple, but very accurate, approximation to the solution of the one-loop evolution equation with flat ${ }^{1}$ inputs and which is valid in the region of small $x(x \lesssim 0.01)$. They also showed that two-loop evolution with flat inputs starting at $Q_{I}^{2} \approx 2 \mathrm{GeV}^{2}$ could fit the data available at that time very well [11].

This state of affairs left scope for argument about the real underlying physics describing the small- $x$ behaviour of $\mathcal{F}_{2}\left(x, Q^{2}\right)$. Those who used Altarelli-Parisi evolution from small scales could be accused of working in regions where perturbation theory was questionable and, perhaps more importantly, of ignoring terms of higher order in $\alpha_{s}$ (but also higher order in $\ln (1 / x)$ ), which

1 Parton inputs behaving like $x^{-1}$, as opposed to $x^{-1-\lambda}$, are referred to as flat, i.e. the parton density rescaled by $x$ is flat. 
seemed from the BFKL equation to have very important effects. Conversely, those who used the BFKL approach could be accused of ignoring all but the leading $-\ln (1 / x)$ terms (and hence ignoring the large $-x$ data) and also of working in a less well-defined theoretical framework than the renormalization group approach based on the factorization of collinear singularities [12]. Starting from an input for the parton distributions with $\lambda \sim 0.25$ at values of $Q_{I}^{2} \sim 4 \mathrm{GeV}^{2}$ was taking the best of both worlds. However, this lacked a real justification for the choice of input, which was significantly steeper than that expected from non-perturbative physics, but rather smaller than that from the BFKL equation, and also ignored potentially important $\ln (1 / x)$ terms in the evolution.

A significant step forward in the investigation of small- $x$ structure functions was the development of the $k_{T}$-factorization theorem [13][14], the prescription for the way in which an off-shell photon-gluon scattering amplitude can be convoluted with the unintegrated gluon Green's function calculated using the BFKL equation to provide the small- $x$ structure functions themselves. Hence, it enables one to find effective moment-space coefficient functions within the BFKL framework. Numerical calculations performed using this method were able to match the available data in a qualitative manner [15], as did similar calculations [16] using a modification of the BFKL equation, i.e. the CCFM equation [17]. The $k_{T}$-factorization formula was also shown to be very important if one insisted on working within the rigorous framework of the traditional renormalization group approach [18]. By showing how $k_{T}$-factorization fits within the collinear factorization framework, Catani and Hautmann were able to calculate all the renormalization group anomalous dimensions to lowest nontrivial order in $\alpha_{s}$ for each power of $\ln (1 / x)$, and similarly for a number of coefficient functions. They demonstrated that within the renormalization group framework the Mellin transformations of the splitting functions, the anomalous dimensions $\gamma_{g g}^{0}\left(N, Q^{2}\right)$ and $\gamma_{g f}^{0}\left(N, Q^{2}\right)$ were the same renormalization-scheme-independent expressions as the effective anomalous dimensions [19] given by the BFKL equation. i.e.

$$
\gamma_{g g}^{0}\left(\alpha_{s}\left(Q^{2}\right) / N\right)=\sum_{m=1}^{\infty} a_{0, m}\left(\frac{\bar{\alpha}_{s}\left(Q^{2}\right)}{N}\right)^{m}, \quad \gamma_{g f}^{0}\left(\alpha_{s}\left(Q^{2}\right) / N\right)=\frac{4}{9} \gamma_{g g}^{0}\left(\alpha_{s}\left(Q^{2}\right) / N\right)
$$

where $\gamma_{g g}^{0}\left(\alpha_{s}\left(Q^{2}\right) / N\right)$ is given by the iterative solution of

$$
1=\frac{\bar{\alpha}_{s}\left(Q^{2}\right)}{N} \chi\left(\gamma_{g g}^{0}\right), \quad \chi(\gamma)=2 \psi(1)-\psi(\gamma)-\psi(1-\gamma)
$$

and the solution as a power series in $\bar{\alpha}_{s}\left(Q^{2}\right) / N$ exists for $|N| \geq \lambda\left(\bar{\alpha}_{s}\left(Q^{2}\right)\right)$. They also derived expressions for $\gamma_{f f}^{1}\left(\alpha_{s}\left(Q^{2}\right) / N\right.$ ) and $\gamma_{f g}^{1}\left(\alpha_{s}\left(Q^{2}\right) / N\right)$ (series of the same form as in (1.1) but with an extra power of $\left.\alpha_{s}\right)$ in certain factorization schemes $\left(\gamma_{f f}^{0}\left(\alpha_{s}\left(Q^{2}\right) / N\right)\right.$ and $\gamma_{f g}^{0}\left(\alpha_{s}\left(Q^{2}\right) / N\right)$ being zero), and also for the coefficient functions $C_{L, 1}^{g}\left(\alpha_{s}\left(Q^{2}\right) / N\right), C_{L, 1}^{f}\left(\alpha_{s}\left(Q^{2}\right) / N\right), C_{2,1}^{g}\left(\alpha_{s}\left(Q^{2}\right) / N\right)$ and $C_{2,1}^{f}\left(\alpha_{s}\left(Q^{2}\right) / N\right)$ (all zeroth-order quantities being zero except $C_{2,0}^{f}$, which is unity). This 
facilitated calculations of structure functions within the normal renormalization group framework, but including much of what is often called BFKL physics (i.e. the leading- $\ln (1 / x)$ terms), and indeed, a number of calculations were performed using somewhat different approaches [20]-[23], and in most cases comparisons with data made. It seemed that by including these terms it was not possible to improve upon the best fits for the small- $x$ data using one- or two-loop evolution from soft inputs [20][22]. Indeed, many ways of including them made the fits significantly worse, and this seemed to be universally true if the fits were more global, i.e. constrained by large $-x$ data [20]. Also, it seemed that there was a very strong dependence on the factorization scheme used to perform the calculations when including the leading- $\ln (1 / x)$ terms [20][22][24][25], and a number of new factorization schemes were invented.

The high precision of the most recent HERA data constrains theory far more than previously, and has changed the above picture somewhat. The best recent global fits seem to come from those intermediate approaches which use NLO perturbation theory with a quite steep (and completely unexplained) input for the singlet quark with $\lambda \sim 0.2$ and a very $Q_{I}^{2}$-sensitive small- $x$ input for the gluon [26]. Fixed order perturbation theory using flat or valence-like inputs and low $Q_{I}^{2}$ fails at the lowest $x$ values, and relatively steep inputs for the singlet quark, i.e. $\lambda \gtrsim 0.2$, seem to be absolutely necessary [27]. Approaches including the leading- $\ln (1 / x)$ terms seemed to fail [28][29] in practically all factorization schemes.

In this paper we will take issue with the above conclusions. In particular we will demonstrate that the apparent failure of approaches using the leading- $\ln (1 / x)$ terms, and certainly the factorization scheme dependence, is due to incorrect methods of incorporating these terms. We will show that the correct leading-order, renormalization-scheme-consistent (RSC) calculation of the structure functions is quite naturally factorization-scheme-independent, and also naturally includes leading- $\ln (1 / x)$ terms in a well-defined form as well as terms leading-order in $\alpha_{s}$. We will also show that this calculation is clearly preferred by data. ${ }^{2}$

This paper will be structured as follows. We begin by defining conventions and giving a brief outline of the different possible types of scheme dependence in the calculation of structure functions. We then quickly review the work of Catani, who has already shown how to obtain factorizationscheme-invariant results in the small- $x$ expansion by writing evolution equations in terms of the physical quantities, the structure functions and "physical anomalous dimensions", rather than in terms of parton densities and of the usual anomalous dimensions [31]. We then give a description of the calculation, within moment space, of structure functions in various expansion schemes using the normal parton language. The first part of this, regarding the normal loop expansion, will highlight points usually not discussed, particularly the form of the inputs. The second part, discussing

2 We note that a very brief presentation of the complete RSC calculation of structure functions has already appeared in [30]. 
the leading $-\ln (1 / x)$ expansion, will present the only correct way to perform this expansion. One finds that by calculating physical quantities in a well-ordered manner the physical anomalous dimensions automatically appear, but not in the form one would obtain by simply solving the evolution equations using these anomalous dimensions at some given order. Moreover, there is a certain degree of predictive power for the form of the inputs for the structure functions at small $x$. We also explain why the standard solutions using the small- $x$ expansions are strongly factorization scheme dependent. To conclude this section we present the argument that there is a unique renormalization-scheme-consistent calculation of structure functions, which applies to both large and small $x$. We present this calculation for both the currently academic case of the nonsinglet structure functions and for the phenomenologically important case of the singlet structure functions. We then briefly discuss how we move from moment space and obtain our $x$-space solutions, and the qualitative form these solutions must take, i.e. our best attempt at predictions. After this long theoretical presentation we consider the comparison with experiment, fitting the data for $\mathcal{F}_{2}\left(x, Q^{2}\right)$ using the renormalization-scheme-consistent solutions, and comparing to global fits using the normal loop expansion at NLO. We conclude that the full renormalization-scheme-consistent calculation gives the best global fit to structure function data, particularly at small $x$. Finally we investigate the phenomenological consequences for $\mathcal{F}_{L}\left(x, Q^{2}\right)$, and also preliminary indications for the charm structure function. ${ }^{3}$

\section{Scheme and Scale Choices.}

For simplicity we work in moment-space for much of this paper, i.e. define the moment-space structure functions by the Mellin transform, i.e.

$$
F\left(N, Q^{2}\right)=\int_{0}^{1} x^{N-1} \mathcal{F}\left(x, Q^{2}\right) d x .
$$

The moment-space coefficient function is defined similarly but, as with the definition of the anomalous dimension, we define the moment space expression for the parton distribution as the Mellin transform of a rescaled parton density i.e

$$
f\left(N, Q^{2}\right)=\int_{0}^{1} x^{N} \mathrm{f}\left(x, Q^{2}\right) d x .
$$

The most general moment-space expression for a structure function is the sum of the products of the expressions for hard scattering with a certain parton (the coefficient functions) with the corresponding, intrinsically nonperturbative parton distributions, ${ }^{4}$

$$
F\left(N, Q^{2}\right)=\sum_{a} C^{a}\left(N, \alpha_{s}\left(\mu_{R}^{2}\right), Q^{2} / \mu_{F}^{2}, \mu_{R}^{2} / \mu_{F}^{2}\right) f_{a}\left(N, \alpha_{s}\left(\mu_{R}^{2}\right), \mu_{R}^{2} / \mu_{F}^{2}\right),
$$

3 There is a rather longer version of this paper which includes much more detail of a pedagogical nature and more comparison with other approaches [32]

4 We deal with the heavy quark thresholds in a rather naive manner, i.e. the quarks are taken to be massless, with a particular flavour becoming active only above a certain $Q^{2}$. 
where $\mu_{F}$ is a factorization scale separating the ultraviolet physics from the soft infrared physics. The coupling $\alpha_{s}\left(\mu_{R}^{2}\right)$ satisfies the renormalization group equation,

$$
\frac{d \alpha\left(\mu_{R}^{2}\right)}{d \ln \left(\mu_{R}^{2}\right)}=-\sum_{n=0}^{\infty} b_{n} \alpha^{n+2}\left(\mu^{2}\right) \equiv-\beta\left(\alpha\left(\mu_{R}^{2}\right)\right)
$$

where $\mu_{R}$ is the renormalization scale, the scale at which the coupling is defined.

Two of the choices left open in the above expressions are common to all perturbative calculations in quantum field theory: the choice of renormalization scheme (the choice of which expansion parameter is to be used, and consequently the form of the perturbative expansion), and subsequently the choice of renormalization scale. Conventionally the $\overline{\mathrm{MS}}$ renormalization scheme is used, along with the simple choice $\mu_{R}=\mu_{F}$, and indeed, the data seem to favour this choice of scale [33]. Hence, we take this simple choice. The remaining ambiguities are due to the particular problems in calculating quantities in QCD, i.e separating the physical quantity into the perturbative coefficient function and the intrinsically nonperturbative parton distribution. We have the freedom of choosing the factorization scale $\mu_{F}$. As with renormalization scheme dependence this does not affect the all-orders calculation and invariance under this choice of scale leads to the evolution equation

$$
\frac{d f_{a}\left(\mu_{F}^{2}\right)}{d \ln \mu_{F}^{2}}=\sum_{b} \gamma_{a b}\left(\alpha_{s}\left(\mu_{F}^{2}\right)\right) f_{b}\left(\mu_{F}^{2}\right)
$$

It is desirable to choose $\mu_{F}^{2}$ to be large in order to make the expansion parameter $\alpha_{s}\left(\mu_{F}^{2}\right)$ small, and to sum large logarithms in $Q^{2}$, and $\mu_{F}^{2}$ is nearly always chosen to be equal to the hard scattering scale $Q^{2}$. We shall also make this simple choice.

This leaves us with our defining equations

$$
F\left(N, Q^{2}\right)=\sum_{a} C^{a}\left(N, \alpha_{s}\left(Q^{2}\right)\right) f_{a}\left(N, Q^{2}\right) \quad \text { and } \quad \frac{d f_{a}\left(Q^{2}\right)}{d \ln Q^{2}}=\sum_{b} \gamma_{a b}\left(\alpha_{s}\left(Q^{2}\right)\right) f_{b}\left(Q^{2}\right)
$$

or being more careful there are two independent structure functions $F_{2}\left(N, Q^{2}\right)$ and $F_{L}\left(N, Q^{2}\right)$. In general we may write

$$
F_{i}\left(N, Q^{2}\right)=\frac{1}{N_{f}}\left(\sum_{j=1}^{N_{f}} e_{j}^{2}\right) F_{i}^{S}\left(N, Q^{2}\right)+F_{i}^{N S}\left(N, Q^{2}\right) \quad(i=2, L),
$$

where the singlet and nonsinglet structure functions are defined by

$$
\begin{gathered}
F_{i}^{S}\left(N, Q^{2}\right)=C_{i}^{f}\left(N, \alpha_{s}\right) f^{S}\left(N, Q^{2}\right)+C_{i}^{g}\left(N, \alpha_{s}\right) g\left(N, Q^{2}\right), \\
F_{i}^{N S}\left(N, Q^{2}\right)=C_{i}^{N S}\left(N, \alpha_{s}\right) \sum_{j=1}^{N_{f}} e_{j}^{2} f_{q_{j}}^{N S}\left(N, Q^{2}\right),
\end{gathered}
$$


where $N_{f}$ is the number of active quark flavours, $f^{S}\left(N, Q^{2}\right)$ and $f_{q_{j}}^{N S}\left(N, Q^{2}\right)$ are the singlet and nonsinglet quark distribution functions respectively, and $g\left(N, Q^{2}\right)$ is the gluon distribution. The equations for the nonsinglet distributions are ordinary differential equations,

$$
\frac{d f_{q_{j}}^{N S}\left(N, Q^{2}\right)}{d \ln Q^{2}}=\gamma_{N S}\left(N, \alpha_{s}\right) f_{q_{j}}^{N S}\left(N, Q^{2}\right),
$$

while those for the singlet sector are coupled

$$
\frac{d}{d \ln Q^{2}}\left(\begin{array}{c}
f^{S}\left(N, Q^{2}\right) \\
g\left(N, Q^{2}\right)
\end{array}\right)=\left(\begin{array}{cc}
\gamma_{f f}\left(N, \alpha_{s}\right) & \gamma_{f g}\left(N, \alpha_{s}\right) \\
\gamma_{g f}\left(N, \alpha_{s}\right) & \gamma_{g g}\left(N, \alpha_{s}\right)
\end{array}\right)\left(\begin{array}{c}
f^{S}\left(N, Q^{2}\right) \\
g\left(N, Q^{2}\right)
\end{array}\right)
$$

This still leaves us one more freedom in our calculation, i.e. how we choose to remove the infrared divergences from the bare coefficient functions and hence how we define our parton distributions. Starting from any particular choice for the definition of parton distributions one may always choose a new set of parton distributions by an invertible transformation

$$
\breve{f}_{a}\left(N, Q^{2}\right)=\sum_{b} U_{a b}\left(N, \alpha_{s}\right) f_{b}\left(N, Q^{2}\right)
$$

where $U_{a b}\left(N, \alpha_{s}\right)$ has a power series expansion in $\alpha_{s}$ such that $U_{a b}\left(N, \alpha_{s}\right)=\delta_{a b}+\mathcal{O}\left(\alpha_{s}\right)$. The structure functions will clearly be unchanged as long as the coefficient functions obey the transformation rule

$$
\breve{C}^{a}\left(N, \alpha_{s}\right)=\left(U^{T}\right)_{a b}^{-1}\left(N, \alpha_{s}\right) C^{b}\left(N, \alpha_{s}\right) .
$$

By substituting $f_{a}\left(N, Q^{2}\right)=\sum_{b} U_{a b}^{-1}\left(N, \alpha_{s}\right) \breve{f}_{b}\left(N, Q^{2}\right)$ into (2.5) we easily find that the new parton densities evolve according to the standard evolution equations but with the new anomalous dimensions

$$
\breve{\gamma}_{a b}\left(N, \alpha_{s}\right)=\sum_{c} \sum_{d} U_{a, c}\left(N, \alpha_{s}\right) \gamma_{c d}\left(N, \alpha_{s}\right) U_{d b}^{-1}\left(N, \alpha_{s}\right)+\sum_{c} \beta\left(\alpha_{s}\right) \frac{\partial U_{a c}\left(N, \alpha_{s}\right)}{\partial \alpha_{s}} U_{c b}^{-1}\left(N, \alpha_{s}\right)
$$

The transformation defined above is called a change of factorization scheme. ${ }^{5}$ However, it is important to realize that, unlike the changes in renormalization scheme, a change in factorization scheme leaves the expression for the structure functions unchanged not only to all orders, but order by order in $\alpha_{s}$. Calculations performed carefully at a given well-defined order in one scheme will lead to precisely the same results for the structure functions as those in another scheme. Also, the coupling constant to be used depends only on the ultraviolet renormalization. We will illustrate this point in $\S 4$. However, we will first discuss Catani's recent proposal for the construction of factorization-scheme-independent structure functions.

5 The matrix $U$ must obey a number of conditions in order that physical requirements on the parton distributions are maintained, e.g. flavour and charge conjugation invariance, fermion number conservation and longitudinal momentum conservation (see for example the second of [18]). However, none of these needs to be satisfied simply in order to keep the structure functions unchanged. 


\section{Evolution Equations for Structure Functions.}

It is, as Catani noticed [31], very simple to obtain factorization-scheme-independent factorization-scheme-independent effective anomalous dimensions governing the evolution of the structure functions In order to obtain these effective anomalous dimensions all one has to do is eliminate the parton densities from the equations (2.6) and (2.5). In order to demonstrate this, let us first consider the simple case of the nonsinglet structure function $F_{2}^{N S}\left(N, Q^{2}\right)$. Multiplying both sides of (2.10) by $\sum_{j=1}^{N_{f}} e_{j}^{2}$ we can clearly write

$$
\frac{d}{d \ln Q^{2}}\left(\frac{F_{2}^{N S}\left(N, Q^{2}\right)}{C_{2}^{N S}\left(N, \alpha_{s}\right)}\right)=\gamma_{N S}\left(N, \alpha_{s}\right) \frac{F_{2}^{N S}\left(N, Q^{2}\right)}{C_{2}^{N S}\left(N, \alpha_{s}\right)}
$$

which becomes the factorization-scheme-independent equation

$$
\frac{d F_{2}^{N S}\left(N, Q^{2}\right)}{d \ln Q^{2}}=\Gamma_{2, N S}\left(N, \alpha_{s}\right) F_{2}^{N S}\left(N, Q^{2}\right)
$$

where $\Gamma_{2, N S}\left(N, \alpha_{s}\right)=\gamma_{N S}\left(N, \alpha_{s}\right)+d \ln \left(C_{2}^{N S}\left(N, \alpha_{s}\right)\right) / d \ln Q^{2}$. Therefore, we have an effective anomalous dimension governing the evolution of each of the nonsinglet structure functions, and clearly $\Gamma_{i}^{N S}\left(N, \alpha_{s}\right)$ must be a factorization-scheme-independent quantity (and is in principle measurable). The solution to this equation is trivial:

$$
F_{2}^{N S}\left(N, Q^{2}\right)=F_{2}^{N S}\left(N, Q_{I}^{2}\right) \exp \left[\int_{\ln Q_{I}^{2}}^{\ln Q^{2}} \Gamma_{2, N S}\left(N, \alpha_{s}\right) d \ln q^{2}\right]
$$

The situation for the singlet structure functions is more complicated. However, using (2.8) for $i=2, L$ we may solve for the parton densities in terms of the two structure functions and the coefficient functions. Substituting these into (2.11) we then obtain the coupled evolution equations

$$
\frac{d}{d \ln Q^{2}}\left(\begin{array}{c}
F_{2}^{S}\left(N, Q^{2}\right) \\
F_{L}^{S}\left(N, Q^{2}\right)
\end{array}\right)=\left(\begin{array}{cc}
\check{\Gamma}_{22}\left(N, \alpha_{s}\right) & \check{\Gamma}_{2 L}\left(N, \alpha_{s}\right) \\
\check{\Gamma}_{L 2}\left(N, \alpha_{s}\right) & \check{\Gamma}_{L L}\left(N, \alpha_{s}\right)
\end{array}\right)\left(\begin{array}{c}
F_{2}^{S}\left(N, Q^{2}\right) \\
F_{L}^{S}\left(N, Q^{2}\right)
\end{array}\right)
$$

The expressions for the physical anomalous dimensions, $\check{\Gamma}_{22}\left(N, \alpha_{s}\right), \check{\Gamma}_{2 L}\left(N, \alpha_{s}\right)$, etc., are straightforward to derive in terms of anomalous dimensions and coefficient functions in any particular factorization scheme using the above procedure, but result in rather cumbersome expressions. It is simplest first to define a factorization scheme such that $F_{2}^{S}\left(N, Q^{2}\right)=f^{S}\left(N, Q^{2}\right)$, i.e. $C_{2}^{f}\left(N, \alpha_{s}\right)=1$, $C_{2}^{g}\left(N, \alpha_{s}\right)=0$ (this is generally known as a DIS type scheme $\left.[34]^{6}\right)$. In terms of the coefficient

6 We call it a DIS "type" scheme because satisfying the above requirement still leaves freedom in how we may define the gluon density, and thus we are still considering a family of schemes. 
functions and anomalous dimensions in this type of scheme we have

$$
\begin{aligned}
\check{\Gamma}_{22}\left(N, \alpha_{s}\right) & =\gamma_{f f}\left(N, \alpha_{s}\right)-\frac{C_{L}^{f}\left(N, \alpha_{s}\right)}{C_{L}^{g}\left(N, \alpha_{s}\right)} \gamma_{f g}\left(N, \alpha_{s}\right) \\
\check{\Gamma}_{2 L}\left(N, \alpha_{s}\right) & =\frac{\gamma_{f g}\left(N, \alpha_{s}\right)}{C_{L}^{g}\left(N, \alpha_{s}\right)} \\
\check{\Gamma}_{L, 2}\left(N, \alpha_{s}\right) & =C_{L}^{g}\left(N, \alpha_{s}\right) \gamma_{g f}\left(N, \alpha_{s}\right)-C_{2}^{f}\left(N, \alpha_{s}\right) \gamma_{g g}\left(N, \alpha_{s}\right)+\frac{d C_{L}^{f}\left(N, \alpha_{s}\right)}{d \ln Q^{2}}+C_{L}^{f}\left(N, \alpha_{s}\right) \gamma_{f f}\left(N, \alpha_{s}\right) \\
& -C_{L}^{f}\left(N, \alpha_{s}\right) \frac{d \ln \left(C_{L}^{g}\left(N, \alpha_{s}\right)\right)}{d \ln Q^{2}}-\frac{\left(C_{L}^{f}\left(N, \alpha_{s}\right)\right)^{2}}{C_{L}^{g}\left(N, \alpha_{s}\right)} \gamma_{f g}\left(N, \alpha_{s}\right), \\
\check{\Gamma}_{L L}\left(N, \alpha_{s}\right) & =\gamma_{g g}\left(N, \alpha_{s}\right)+\frac{d \ln \left(C_{L}^{g}\left(N, \alpha_{s}\right)\right)}{d \ln Q^{2}}+\frac{C_{L}^{f}\left(N, \alpha_{s}\right)}{C_{L}^{g}\left(N, \alpha_{s}\right)} \gamma_{f g}\left(N, \alpha_{s}\right) .
\end{aligned}
$$

Before going any further let us remark that we believe there is a (purely technical) problem with the above expression. As is well known $F_{L}\left(N, Q^{2}\right)$ starts at an order of $\alpha_{s}$ higher than $F_{2}\left(N, Q^{2}\right)$. Because of this there is an intrinsic asymmetry in the above definitions, with $\check{\Gamma}_{2 L}\left(N, \alpha_{s}\right)$ beginning at zeroth order in $\alpha_{s}, \check{\Gamma}_{22}\left(N, \alpha_{s}\right)$ and $\check{\Gamma}_{L L}\left(N, \alpha_{s}\right)$ beginning at first order, and $\Gamma_{L 2}\left(N, \alpha_{s}\right)$ beginning at second order. This asymmetry leads to the results obtained by solving these equations order by order being different that those using the normal parton picture even in the loop expansion. A trivial modification of Catani's approach is therefore to accept that $F_{L}\left(N, Q^{2}\right)$ contains an extra power of $\alpha_{s}$, and to define the new structure function $\hat{F}_{L}\left(N, Q^{2}\right)=F_{L}\left(N, Q^{2}\right) /\left(\alpha_{s} /(2 \pi)\right)$. The longitudinal coefficient functions are likewise changed to $\hat{C}_{L}^{a}\left(N, \alpha_{s}\right)=C_{L}^{a}\left(N, \alpha_{s}\right) /\left(\alpha_{s} /(2 \pi)\right)$, and the singlet evolution equations become

$$
\frac{d}{d \ln Q^{2}}\left(\begin{array}{c}
F_{2}^{S}\left(N, Q^{2}\right) \\
\hat{F}_{L}^{S}\left(N, Q^{2}\right)
\end{array}\right)=\left(\begin{array}{ll}
\Gamma_{22}\left(N, \alpha_{s}\right) & \Gamma_{2 L}\left(N, \alpha_{s}\right) \\
\Gamma_{L 2}\left(N, \alpha_{s}\right) & \Gamma_{L L}\left(N, \alpha_{s}\right)
\end{array}\right)\left(\begin{array}{c}
F_{2}^{S}\left(N, Q^{2}\right) \\
\hat{F}_{L}^{S}\left(N, Q^{2}\right)
\end{array}\right)
$$

where the $\Gamma\left(N, \alpha_{s}\right)$ 's are defined precisely as in (3.5), but in terms of of $\hat{C}_{L}^{a}\left(N, \alpha_{s}\right)$ rather than $C_{L}^{a}\left(N, \alpha_{s}\right)$. This procedure restores the symmetry between the physical anomalous dimensions, and makes the order-by-order-in- $\alpha_{s}$ calculations essentially the same as when using evolution of parton distributions. It is, of course, trivial to obtain the physical $F_{L}\left(N, Q^{2}\right)$ from $\hat{F}_{L}\left(N, Q^{2}\right) .^{7}$

Having made our redefinition of the quantities with which we work, we now have a direct relationship between possible calculations using the evolution equations for structure functions and the solutions using the parton densities. At present the parton anomalous dimensions and coefficient functions are known to order $\alpha_{s}^{2}$. It is easy to see that this allows us to derive each of the $\Gamma$ 's to order $\alpha_{s}^{2}$. Similarly, from the known expansions of the parton anomalous dimensions and coefficient functions in the form $\alpha_{s}^{n} \sum_{m=1-n}^{\infty} a_{m}\left(\alpha_{s} / N\right)^{m}[18]$, we can calculate $\Gamma_{L L}^{0}\left(N, \alpha_{s}\right)$ and $\Gamma_{L 2}^{0}\left(N, \alpha_{s}\right)$, $\Gamma_{2 L}^{0}\left(N, \alpha_{s}\right)$ and $\Gamma_{22}^{0}\left(N, \alpha_{s}\right)$ (where both are trivially zero), and $\Gamma_{2 L}^{1}\left(N, \alpha_{s}\right)$ and $\Gamma_{22}^{1}\left(N, \alpha_{s}\right)$. This is

\footnotetext{
7 Similarly, it is also best to work with the rescaled nonsinglet structure function $\hat{F}_{L}^{N S}\left(N, Q^{2}\right)$.
} 
the same order as for the parton anomalous dimensions, with the longitudinal anomalous dimensions having similar structure to the gluon anomalous dimensions and the $\Gamma_{2 a}\left(N, \alpha_{s}\right)$ 's having similar structure to the quark anomalous dimensions. The resulting expressions are relatively simple, being

$$
\begin{aligned}
\Gamma_{22}^{1}\left(\alpha_{s} / N\right) & =-\frac{1}{(2 \pi)}\left(\hat{C}_{L, 1,0}^{f}-\frac{4}{9} \hat{C}_{L, 1,0}^{g}\right)\left(\frac{3}{2} \gamma_{g g}^{0}\left(\alpha_{s} / N\right)+\sum_{n=0}^{\infty}\left(\gamma_{g g}^{0}\left(\alpha_{s} / N\right)\right)^{n}\right) \\
& -\frac{4}{9} \gamma_{f g}^{1,0}\left(N, \alpha_{s}\right), \\
\Gamma_{2 L}^{1}\left(\alpha_{s} / N\right) & =\frac{1}{(2 \pi)}\left(\frac{3}{2} \gamma_{g g}^{0}\left(\alpha_{s} / N\right)+\sum_{n=0}^{\infty}\left(\gamma_{g g}^{0}\left(\alpha_{s} / N\right)\right)^{n}\right), \\
\Gamma_{L, 2}^{0}\left(N, \alpha_{s}\right) & =-\left(\hat{C}_{L, 1,0}^{f}-\frac{4}{9} \hat{C}_{L, g}^{1,0}\right) \gamma_{g g}^{0}\left(\alpha_{s} / N\right), \\
\Gamma_{L L}^{0}\left(\alpha_{s} / N\right) & =\gamma_{g g}^{0}\left(\alpha_{s} / N\right),
\end{aligned}
$$

where $\gamma_{f g}^{1,0}, C_{L, 1,0}^{f}$ and $C_{L, 1,0}^{g}$ are the one-loop contributions to $\gamma_{f g}^{1}\left(\alpha_{s} / N\right), C_{L, 1}^{f}\left(\alpha_{s} / N\right)$ and $C_{L, 1}^{g}\left(\alpha_{s} / N\right)$ respectively. Each of these anomalous dimensions is renormalization scheme invariant as well as factorization scheme invariant, as we would expect for leading-order physical quantities.

Solving the evolution equations for the structure functions using any subset of the currently known physical anomalous dimensions guarantees a result which is factorization scheme independent. However, there is considerable freedom in how we may solve the equations. We could, for example, simply put all of the anomalous dimensions currently known into (3.6) and then find the whole solution. Alternatively, we could solve using just the order $\alpha_{s}$ anomalous dimensions and then try to perturb about this solution in an ordered manner. These two approaches would lead to rather different answers, but both would be factorization scheme independent. The problem of obtaining a correctly ordered solution for the structure function will be discussed in detail in the next section. We will initially use the familiar parton distributions and coefficient functions, and show that, even when using this approach, if we solve producing a well-defined expansion for the structure functions, we constrain the form of the solution rigidly and thus automatically avoid the problem of factorization scheme dependence. In fact, not only do we obtain the physical anomalous dimensions, but also find precisely how we should use them.

\section{Ordered Calculations of Structure Functions.}

There are in principle many different expansion methods one may use when obtaining solutions for the structure functions. The standard one is simply solving order by order in $\alpha_{s}$. But there is also the expansion in leading powers of $\ln (1 / x)$ for given powers in $\alpha_{s}$ (or equivalently in powers of $N^{-1}$ in moment space), as we have already discussed. One can also combine the two expansion methods, and indeed, we will later argue that this is the only correct thing to do. Nevertheless, we will begin by outlining the procedure for making a well-ordered calculation of structure functions using the standard loop expansion. Although this is well known, we feel it is worth presenting it briefly, and making some points which are not usually highlighted, especially concerning the role of the starting scale. We may then discuss the more complicated cases of the expansion in leading powers of $\ln (1 / x)$ and the combined expansion. 


\subsection{Loop Expansion.}

We begin by introducing some new notation. In order to solve the evolution equations for the parton densities order by order in $\alpha_{s}$ and hence obtain expressions for $F_{i}^{N S}\left(N, Q^{2}\right)$ and $F_{i}^{S}\left(N, Q^{2}\right)$ we make use of equation (2.4) to rewrite the evolution equation for the nonsinglet parton density as

$$
\alpha_{s}^{2}\left(Q^{2}\right) \frac{d f_{q_{j}}^{N S}\left(N, Q^{2}\right)}{d \alpha_{s}\left(Q^{2}\right)}=-\tilde{\gamma}_{N S}\left(N, \alpha_{s}\right) f_{q_{j}}^{N S}\left(N, Q^{2}\right)
$$

where $\tilde{\gamma}_{N S}\left(N, \alpha_{s}\right)=\alpha_{s}^{2}\left(Q^{2}\right) \gamma_{N S}\left(N, \alpha_{s}\right) / \beta\left(\alpha_{s}\right)$, and similarly for the singlet distributions, with similar definitions for $\tilde{\gamma}_{f f}\left(N, \alpha_{s}\right)$ etc. as for $\tilde{\gamma}_{N S}\left(N, \alpha_{s}\right)$. Each of the $\tilde{\gamma}$ 's may now be written as

$$
\tilde{\gamma}\left(N, \alpha_{s}\right)=\sum_{n=0}^{\infty} \tilde{\gamma}^{n, l}(N) \alpha_{s}^{n+1}
$$

where where the super-subscript $n, l$ denotes the $(n+1)$-loop quantity and we have an analogous definition for the normal anomalous dimensions.

Using our definition, the evolution equations may be solved order by order in $\alpha_{s}$. When doing this it is necessary to choose a starting scale $Q_{I}^{2}$ for the perturbative evolution of the parton distributions (or equivalently a starting value of the coupling $\alpha_{s}\left(Q_{I}^{2}\right)$ ), and specify input parton distributions at this scale. Let us discuss the choice of this scale briefly. $Q_{I}^{2}$ must clearly be chosen to be large enough that perturbative evolution should be reliable, i.e. $\alpha_{s}\left(Q^{2}\right) \lesssim 0.3$ and also such that "higher twist" $\left(\Lambda_{Q C D}^{2} / Q^{2}\right)$ corrections should be very small. Traditionally evolution only takes place up from this starting scale, for the simple reason of convenience, and also because some form of the inputs has been expected at low starting scales. Until a few years ago the requirements described above led to a choice of $Q_{I}^{2} \approx 4 \mathrm{GeV}^{2}$. In the past couple of years this value has often been chosen to be rather lower, due to the apparent success of evolution from lower starting scales, and also because much of the interesting small- $x$ data is now at $Q^{2} \leq 4 \mathrm{GeV}^{2}$. These choices have been accompanied by guesses for the form of the inputs at low starting scales, e.g valence-like [9], or flat [5][10]. We make no assumptions of the above sort about the value of $Q_{I}^{2}$. We require it to be high enough to be in the perturbative regime and to avoid higher twists, but acknowledge that there is no reason why $Q_{I}^{2}$ cannot be chosen to be quite large, and evolution away from the starting scale performed both up and down in $Q^{2}$. Taking this open-minded approach we also assume that only perturbative effects can lead to deviations from soft behaviour of the structure functions, and also demand that the form of our well-ordered expressions for the structure functions is as insensitive as possible to this choice, thus making the choice of $Q_{I}^{2}$ as open as possible.

We begin by solving for the nonsinglet parton distributions, which are an easily understandable model. In this case the solution is particularly simple. Integrating both sides of (4.1) we obtain

$$
f_{q_{j}}^{N S}\left(N, Q^{2}\right)=\left[\sum_{k=0}^{\infty} \alpha_{s}^{k}\left(Q_{I}^{2}\right) f_{q_{j}, k}^{N S}\left(N, Q_{I}^{2}\right)\right]\left(\frac{\alpha_{s}\left(Q_{I}^{2}\right)}{\alpha_{s}\left(Q^{2}\right)}\right)^{\tilde{\gamma}_{N S}^{0, l}(N)} \exp \left[\sum_{n=1}^{\infty} \frac{\left(\alpha_{s}^{n}\left(Q_{I}^{2}\right)-\alpha_{s}^{n}\left(Q^{2}\right)\right)}{n} \tilde{\gamma}_{N S}^{n, l}(N)\right] .
$$


Perhaps unconventionally, we explicitly express the input $f_{q_{j}}^{N S}\left(N, Q_{I}^{2}\right)$ as a power series in $\alpha_{s}\left(Q_{I}^{2}\right)$. This is necessary because changes in the starting scale $Q_{I}^{2}$ lead to $\alpha_{s}\left(Q_{I}^{2}\right)$-dependent changes in the expression for the evolution term, which must be compensated for by $\alpha_{s}\left(Q_{I}^{2}\right)$-dependent changes in the starting distribution in order to leave the whole expression for the parton distributions unchanged, as required. Let us examine this briefly by looking at the change of the lowest-order piece of (4.3), i.e.

$$
f_{q_{j}, 0}^{N S}\left(N, Q^{2}\right)=f_{q_{j}, 0}^{N S}\left(N, Q_{I}^{2}\right)\left(\frac{\alpha_{s}\left(Q_{I}^{2}\right)}{\alpha_{s}\left(Q^{2}\right)}\right)^{\tilde{\gamma}_{N S}^{0, l}(N)},
$$

under a change in starting scale, $Q_{I}^{2} \rightarrow(1+\delta) Q_{I}^{2}$, where $\delta$ is some constant. We may regain expressions in terms of $Q_{I}^{2}$ by expanding the coupling constant in the form

$$
\alpha_{s}\left((1+\delta) Q_{I}^{2}\right)=\alpha_{s}\left(Q_{I}^{2}\right)-\delta b_{0} \alpha_{s}^{2}\left(Q_{I}^{2}\right)+\mathcal{O}\left(\alpha_{s}^{3}\left(Q_{I}^{2}\right)\right)
$$

Under this change in the input coupling constant, the evolution term in (4.4) undergoes a change

$$
\Delta\left(\frac{\alpha_{s}\left(Q_{I}^{2}\right)}{\alpha_{s}\left(Q^{2}\right)}\right)^{\tilde{\gamma}_{N S}^{0, l}(N)}=-\alpha_{s}\left(Q_{I}^{2}\right) \delta b_{0} \tilde{\gamma}_{N S}^{0, l}(N)\left(\frac{\alpha_{s}\left(Q_{I}^{2}\right)}{\alpha_{s}\left(Q^{2}\right)}\right)^{\tilde{\gamma}_{N S}^{0, l}(N)}+\text { higher order in } \alpha_{s}\left(Q_{I}^{2}\right)
$$

This change in the parton distribution due to the variation in the leading term can be countered, up to higher orders, by a change in the order $\alpha_{s}\left(Q_{I}^{2}\right)$ input of the form

$$
\Delta f_{q_{j}, 1}^{N S}\left(N, Q_{I}^{2}\right)=\delta b_{0} \tilde{\gamma}_{N S}^{0, l}(N) f_{q_{j}, 0}^{N S}\left(N, Q_{I}^{2}\right)
$$

Hence, changes in the evolution term due to a change in $Q_{I}^{2}$ begin at first order in $\alpha_{s}\left(Q_{I}^{2}\right)$, and are therefore absorbed by terms in the input at first order and beyond. The zeroth-order input is insensitive to such changes and is $Q_{I}^{2}$-independent: $f_{q_{j}, 0}^{N S}\left(N, Q_{I}^{2}\right) \equiv f_{q_{j}, 0}^{N S}(N)$. Higher-order changes in the parton distributions due to changes in $Q_{I}^{2}$ are all accounted for by changes in the higherorder inputs which are equal to functions of $N$ dependent only on the anomalous dimensions. Therefore, these higher-order inputs consist of perturbative parts multiplying the fundamentally nonperturbative $f_{q_{j}, 0}^{N S}(N)$. As we will soon discuss, there are other constraints to be satisfied, e.g. factorization scheme independence of the input for the structure function, and this slightly complicates the above picture, but does not change the main conclusions.

Thus, it is necessary to express the input as a power series in $\alpha_{s}\left(Q_{I}^{2}\right)$ in order to make the parton distribution $Q_{I}^{2}$-independent ${ }^{8}$, but only one intrinsically nonperturbative input which is

8 Another reason for explicitly writing the input as a power series in $\alpha_{s}\left(Q_{I}^{2}\right)$ is that it makes little sense to demand that the starting distribution should not have a perturbative expansion, unless there is something special about a particular factorization scheme. Any transformation from a scheme in which the starting parton distribution is purely zeroth order in $\alpha_{s}\left(Q_{I}^{2}\right)$ will lead to a nonperturbative starting distribution multiplied by a power series expansion in $\alpha_{s}\left(Q_{I}^{2}\right)$. 
$Q_{I}^{2}$-independent is needed. Usually in analyses of structure functions the parton inputs are taken to be a single $\alpha_{s}\left(Q_{I}^{2}\right)$-independent function which is implicitly allowed to be $Q_{I}^{2}$-dependent. Phenomenologically, this is normally much the same, but we stress the formally correct expression for the input here since it is rather important when constructing properly ordered solutions, and leads to some predictive power, especially in the small $x$ limit.

Substituting our solution for the parton distribution into (2.9), we obtain the general expression for the nonsinglet structure functions,

$$
\begin{array}{r}
F_{i}^{N S}\left(N, Q^{2}\right)=\left[\left(\delta_{i, 2}+\sum_{m=1}^{\infty} C_{i, m, l}^{N S}(N) \alpha_{s}^{m}\left(Q^{2}\right)\right) \sum_{j=1}^{N_{f}} e_{j}^{2} \sum_{k=0}^{\infty} \alpha_{s}^{k}\left(Q_{I}^{2}\right) f_{q_{j}, k}^{N S}\left(N, Q_{I}^{2}\right)\right] \\
\left(\frac{\alpha_{s}\left(Q_{I}^{2}\right)}{\alpha_{s}\left(Q^{2}\right)}\right)^{\tilde{\gamma}_{N S}^{0, l}(N)} \exp \left[\sum_{n=1}^{\infty} \frac{\left(\alpha_{s}^{n}\left(Q_{I}^{2}\right)-\alpha_{s}^{n}\left(Q^{2}\right)\right)}{n} \tilde{\gamma}_{N S}^{n, l}(N)\right] .
\end{array}
$$

It is clear that this may be written as

$$
F_{i}^{N S}\left(N, Q^{2}\right)=\left(\frac{\alpha_{s}\left(Q_{I}^{2}\right)}{\alpha_{s}\left(Q^{2}\right)}\right)^{\tilde{\gamma}_{N S}^{0, l}(N)} \sum_{n=0}^{\infty} \sum_{m=0}^{n} F_{i, n m}^{N S}\left(N, Q_{I}^{2}\right) \alpha_{s}^{n-m}\left(Q_{I}^{2}\right) \alpha_{s}^{m}\left(Q^{2}\right)=\sum_{n=0}^{\infty} F_{i, n}^{N S}\left(N, Q^{2}\right),
$$

and that, once a choice of renormalization scheme and starting scale have been made, each of the $F_{n m}^{N S}(N)$ must be invariant quantities under changes of factorization scheme. Any well-ordered calculation of the structure function should include all complete terms in (4.9) up to a given order in $n$ and $m$, and no partial terms. In practice it is possible to work to a given order in $n$ including all $m \leq n$, i.e. to expand to a given order in powers of $\alpha_{s}\left(Q^{2}\right)$ plus powers of $\alpha_{s}\left(Q_{I}^{2}\right)$, if the $\tilde{\gamma}^{\prime}$ s and $C_{i}$ 's are known to this order.

We shall briefly describe how to construct this ordered solution for the structure functions, working up from zeroth order. Consider first calculating $F_{2}^{N S}\left(N, Q^{2}\right)$ by working to zeroth order in $C_{2}^{N S}\left(N, \alpha_{s}\right), \tilde{\gamma}_{N S}\left(N, \alpha_{s}\right)$ and the starting distribution (remembering that this is $Q_{I}^{2}$-independent). To this order

$$
F_{2,0}^{N S}\left(N, Q^{2}\right)=\sum_{j=1}^{N_{f}} e_{j}^{2} f_{q_{j}, 0}^{N S}(N)\left(\frac{\alpha_{s}\left(Q_{I}^{2}\right)}{\alpha_{s}\left(Q^{2}\right)}\right)^{\tilde{\gamma}_{N S}^{0, l}(N)} .
$$

Using the one-loop expression for the running coupling, as is appropriate for a lowest-order calculation, each of the quantities in this expression is factorization scheme independent and also renormalization scheme independent. Therefore we have a consistent leading-order (LO) expression. If we calculate $F_{L}^{N S}\left(N, Q^{2}\right)$ the zeroth-order coefficient function is zero. However, the only contribution for $n=1$ in (4.9) comes from working to first order in $C_{L}^{N S}\left(N, \alpha_{s}\right)$ and to zeroth order in $\tilde{\gamma}_{N S}\left(N, \alpha_{s}\right)$ and the starting distribution. This leads to the LO expression for $F_{L}^{N S}\left(N, Q^{2}\right)$ of

$$
F_{L, 1}^{N S}\left(N, Q^{2}\right)=\alpha_{s}\left(Q^{2}\right) C_{L, 1, l}^{N S}(N) \sum_{j=1}^{N_{f}} e_{j}^{2} f_{q_{j}, 0}^{N S}(N)\left(\frac{\alpha_{s}\left(Q_{I}^{2}\right)}{\alpha_{s}\left(Q^{2}\right)}\right)^{\tilde{\gamma}_{N S}^{0, l}(N)}
$$


Again, using the one-loop expression for the running coupling, every term in this expression is both factorization scheme and renormalization scheme independent, giving a well-defined LO expression.

We now consider the first correction to these expressions. The first-order expression for the renormalization group equation is

$$
\alpha_{s}\left(Q^{2}\right) \frac{d f_{q_{j}, 1}^{N S}\left(N, Q^{2}\right)}{d \alpha_{s}\left(Q^{2}\right)}=-\tilde{\gamma}_{N S}^{0, l}(N, \alpha, s) f_{q_{j}, 1}^{N S}\left(N, Q^{2}\right)-\alpha_{s}\left(Q^{2}\right) \tilde{\gamma}_{N S}^{1, l}(N) f_{q_{j}, 0}^{N S}\left(N, Q^{2}\right),
$$

with solution

$$
f_{q_{j}, 1}^{N S}\left(N, Q^{2}\right)=\left[\left(\alpha_{s}\left(Q_{I}^{2}\right)-\alpha_{s}\left(Q^{2}\right)\right) \tilde{\gamma}_{N S}^{1, l}(N) f_{q_{j}, 0}^{N S}(N)+\alpha_{s}\left(Q_{I}^{2}\right) f_{q_{j}, 1}^{N S}\left(N, Q_{I}^{2}\right)\right]\left(\frac{\alpha_{s}\left(Q_{I}^{2}\right)}{\alpha_{s}\left(Q^{2}\right)}\right)^{\tilde{\gamma}_{N S}^{0, l}(N)} .
$$

Multiplying $f_{q_{j}, 0}^{N S}$ by $\alpha_{s}\left(Q^{2}\right) C_{2,1, l}^{N S}(N)$ and adding to $f_{q_{j}, 1}^{N S}$, we clearly obtain all terms in the expression (4.9) for $F_{2}^{N S}\left(N, Q^{2}\right)$ ) at $n=1$. Adding this to (4.10) we obtain the factorization-schemeindependent expression for $F_{2}^{N S}\left(N, Q^{2}\right)$ up to $n=1$. We note that this is not the same as finding the complete solution to the renormalization group equation including all terms in the anomalous dimension up to first order in $\tilde{\gamma}$ and multiplying the solution by the coefficient function up to first order. This procedure would involve the exponentiation of the anomalous dimension, and thus would include incomplete parts of the $F_{n m}^{N S}(N)$ 's for $n \geq 1$, and would be a factorization-schemedependent, and hence physically ambiguous quantity.

Similarly to $F_{2}^{N S}\left(N, Q^{2}\right)$ we can obtain the NLO factorization-scheme-independent expression for $F_{L}^{N S}\left(N, Q^{2}\right)$. The expression at $n=2$ is obtained by adding the product of the first-order coefficient function and $f_{q_{j}, 1}^{N S}\left(N, Q^{2}\right)$ to the product of the second-order coefficient function and $f_{q_{j}, 0}^{N S}\left(N, Q^{2}\right)$. The NLO $F_{L}^{N S}\left(N, Q^{2}\right)$ is the sum of this and (4.11). When working to NLO for either structure function we now have expressions which are renormalization scheme dependent. This scheme dependence compensates for the renormalization scheme dependence of the two-loop coupling constant (which has a renormalization-scheme-dependent value for $\Lambda_{Q C D}$ ), and it is this expression for the coupling that we should use at this level. Doing so guarantees the renormalization scheme independence of the structure functions up to corrections of higher order in $\alpha_{s}$, i.e. $\mathcal{O}\left(\alpha_{s}^{2} F_{2(L), 0(1)}^{N S}\right)$.

It is now simple to see how to construct structure functions order by order. Defining the $n_{\text {th }}$ order renormalization group equation by

$$
\alpha_{s}\left(Q^{2}\right) \frac{d f_{q_{j}, n}^{N S}\left(N, Q^{2}\right)}{d \alpha_{s}\left(Q^{2}\right)}=-\sum_{m=0}^{n} \tilde{\gamma}_{N S}^{m, l}(N, \alpha, s) f_{q_{j}, n-m}^{N S}\left(N, Q^{2}\right),
$$

it is easy to prove by induction that the solution contains all terms in the full solution with a given sum of powers of $\alpha_{s}\left(Q^{2}\right)$ and $\alpha_{s}\left(Q_{I}^{2}\right)$ multiplying the everpresent $\left(\alpha_{s}\left(Q_{I}^{2}\right) / \alpha_{s}\left(Q^{2}\right)\right)^{\tilde{\gamma}_{N S}, l}(N)$ factor. Thus, $F_{i, n}^{N S}\left(N, Q^{2}\right)$ is given by

$$
F_{i, n}^{N S}\left(N, Q^{2}\right)=\sum_{j}^{N f} e_{j}^{2} \sum_{m=0}^{n} C_{i, m, l}^{N S} \alpha_{s}^{m}\left(Q^{2}\right) f_{q_{j}, n-m}^{N S}\left(N, Q^{2}\right)
$$


The $n_{\mathrm{th}}$-order scheme-independent structure function is then the sum of the $F_{i, n}^{N S}\left(N, Q^{2}\right)$ up to order $n$. Including all $F_{2, m}^{N S}\left(N, Q^{2}\right)$ up to order $n$ is working to $(n+1)_{\text {th }}$ nontrivial order, and requires the $(n+1)$-loop coupling in order to make the expression renormalization scheme invariant up to higher orders in $\alpha_{s}$. Similarly, including all $F_{L, m}^{N S}\left(N, Q^{2}\right)$ up to order $n$ is working to $n_{\text {th }}$ nontrivial order, and requires the $n$-loop coupling.

This procedure clearly provides factorization scheme independence and renormalization scheme independence for this method of expansion. We can also see how it relates to the discussion of the factorization-scheme-invariant evolution equations in terms of the structure functions. In order to do this let us consider the solution for the non-singlet structure function $F_{2}^{N S}\left(N, Q^{2}\right)$ again. We may rewrite our general solution (4.8) in the form

$$
\begin{aligned}
& F_{2}^{N S}\left(N, Q^{2}\right)=\left[\left(1+\sum_{m=1}^{\infty} C_{2, m, l}^{N S}(N) \alpha_{s}^{m}\left(Q_{I}^{2}\right)\right) \sum_{j=1}^{N_{f}} e_{j}^{2} \sum_{k=0}^{\infty} \alpha_{s}^{k}\left(Q_{I}^{2}\right) f_{q_{j}, k}^{N S}\left(N, Q_{I}^{2}\right)\right]\left(\frac{\alpha_{s}\left(Q_{I}^{2}\right)}{\alpha_{s}\left(Q^{2}\right)}\right)^{\tilde{\gamma}_{N S}^{0, l}(N)} \times \\
& \quad \exp \left[\sum_{n=1}^{\infty} \frac{\left(\alpha_{s}^{n}\left(Q_{I}^{2}\right)-\alpha_{s}^{n}\left(Q^{2}\right)\right)}{n} \tilde{\gamma}_{N S}^{n, l}(N)+\int_{\ln Q_{I}^{2}}^{\ln Q^{2}} \frac{d}{d \ln q^{2}} \ln \left(1+\sum_{m=1}^{\infty} C_{2, m, l}^{N S}(N) \alpha_{s}^{m}\left(q^{2}\right)\right) d \ln q^{2}\right] .
\end{aligned}
$$

This way of writing $F_{2}^{N S}\left(N, Q^{2}\right)$ is particularly useful since it separates the solution into the value of the structure function at $Q_{I}^{2}$ (the term in square brackets), and the ratio of its value at a different $Q^{2}$ with this initial value (the rest of the expression). Clearly these two quantities must be separately factorization scheme independent. Also, it is clear that this solution is of exactly the same form as (3.3), and we can express it simply in terms of an input for $F_{2}^{N S}\left(N, Q^{2}\right)$ at $Q_{I}^{2}$ and a physical anomalous dimension which governs the evolution, i.e. our input and our prediction.

The input and the evolution will mix with each other if we make a change in the starting scale, however. Examining the effects of such a change for the full physical quantity gives us information about the form of the input. The lowest-order input for the structure function is just $F_{2,0}^{N S}(N)=\sum_{j=1}^{N_{f}} e_{j}^{2} f_{q_{j}, 0}^{N S}(N)$. Making the change of starting scale and consequently of $\alpha_{s}\left(Q_{I}^{2}\right)$ already considered, the change in the lowest-order evolution is as in (4.6), and this leads to a change in the lowest-order structure function which is of higher order, and which can be absorbed by a change in the NLO input for the structure function of

$$
\Delta F_{2,1}^{N S}\left(N, Q_{I}^{2}\right)=\delta b_{0} \tilde{\gamma}_{N S}^{0, l}(N) F_{2,0}^{N S}(N)
$$

In terms of parton distributions and coefficient functions

$$
F_{2,1}^{N S}\left(N, Q_{I}^{2}\right)=\sum_{j=1}^{N_{f}} e_{j}^{2}\left(f_{q_{j}, 1}^{N S}\left(N, Q_{I}^{2}\right)+C_{2,1, l}^{N S}(N) f_{q_{j}, 0}^{N S}(N)\right)
$$

We chose the change in $f_{q_{j}, 1}^{N S}\left(N, Q_{I}^{2}\right)$ in (4.7) so that the structure function would be independent of $Q_{I}^{2}$, and it is clear that that is consistent with (4.17) and (4.18). However, we can now say 
more about the form of $f_{q_{j}, 1}^{N S}\left(N, Q_{I}^{2}\right)$. Because it is the leading term in the expression for the input which depends on $\alpha_{s}\left(Q_{I}^{2}\right), F_{2,1}^{N S}\left(N, Q_{I}^{2}\right)$ must be renormalization scheme independent. However, $C_{2,1, l}^{N S}(N)$ is renormalization scheme dependent, so $f_{q_{j}, 1}^{N S}\left(N, Q_{I}^{2}\right)$ must also be renormalization scheme dependent in a way such as to cancel this. Hence, $f_{q_{j}, 1}^{N S}\left(N, Q_{I}^{2}\right)$ must not only have a part like $\ln \left(Q_{I}^{2}\right) \gamma_{N S}^{0, l}(N) f_{q_{j}, 0}^{N S}(N)$ in order to maintain $Q_{I}^{2}$-independence of the structure function, but also a part like $-C_{2,1, l}^{N S}(N) f_{q_{j}, 0}^{N S}(N)$ in order to maintain renormalization scheme independence, i.e.

$$
f_{q_{j}, 1}^{N S}\left(N, Q_{I}^{2}\right)=\left(\ln \left(Q_{I}^{2} / A_{N S}\right) \gamma_{N S}^{0, l}(N)-C_{2,1, l}^{N S}(N)\right) f_{q_{j}, 0}^{N S}(N)
$$

where $A_{N S}$ is some unknown scale parameter. So we see that

$$
F_{2,1}^{N S}\left(N, Q_{I}^{2}\right)=\ln \left(Q_{I}^{2} / A_{N S}\right) \gamma_{N S}^{0, l}(N) F_{2,0}^{N S}(N) \equiv \ln \left(Q_{I}^{2} / A_{N S}\right) \Gamma_{N S}^{0, l}(N) F_{2,0}^{N S}(N)
$$

It is clear that this does not spoil our argument that $f_{q_{j}, 1}^{N S}\left(N, Q_{I}^{2}\right)$ consists of perturbatively calculable quantities multiplying the fundamentally nonperturbative $f_{q_{j}, 0}^{N S}(N)$, and that all the higherorder inputs may be chosen to be perturbative functions multiplying this nonperturbative input, and therefore that the input for the structure function is a perturbative power series (depending on the physical anomalous dimension) multiplying the single nonperturbative factor $f_{q_{j}, 0}^{N S}(N)$, which may also be interpreted as a fundamentally nonperturbative input for the structure function $F_{2,0}^{N S}(N)$. Hence, demanding invariance of our expression for the structure function under changes in $Q_{I}^{2}$ leads us to a power series expression for the input, but with only one (for each quark) really nonperturbative factor for this input. We also see that if $Q_{I}^{2}=A_{N S}$, the first-order perturbative correction to $F_{2}^{N S}\left(N, Q_{I}^{2}\right)$ vanishes. Hence, we might expect $A_{N S}$ to be some scale typical of the transition between perturbative and nonperturbative physics, i.e $A_{N S} \lesssim 1 \mathrm{GeV}$.

Separating the expression for the structure function into a definite input and evolution part also enables us to view the loop expansion in an alternative manner. We see that when expanding out to $n_{\text {th }}$ order in the loop expansion we are including all terms where the order of the input part for the structure function added to the order of the evolution part of the structure function is less than or equal to $n$. Writing the solution as in (4.16) does, however, also illustrate that demanding factorization scheme invariance does not on its own force us into the strictly defined loop expansion. It is clear that we could, if we wished, expand the input and evolution term out to different orders in $\alpha_{s}$, still maintaining factorization scheme independence. However, this is not a sensible approach for reasons of renormalization scheme dependence. If we were to expand out the input and evolution terms to different orders in $\alpha_{s}$ we should really use $\alpha_{s}$ itself calculated to a different order in each case, surely a perverse thing to do. It makes far more sense to combine physical quantities to a given order.

The solution for the longitudinal structure function is much the same as for $F_{2,0}^{N S}\left(N, Q^{2}\right)$. The situation for the singlet structure function is Exactly analogous to that for the nonsinglet, but 
is complicated by the fact that we now have coupled evolution equations for the quark and gluon distributions. This makes it impossible to write a closed form for the solution to the renormalization group equations in the way we did for the nonsinglet case in (4.16), but the equations may be solved order by order in the same way, i.e. the lowest-order solution is

$$
\begin{aligned}
& f_{0}^{S}\left(N, Q^{2}\right)=f_{0}^{S,+}(N)\left(\frac{\alpha_{s}\left(Q_{I}^{2}\right)}{\alpha_{s}\left(Q^{2}\right)}\right)^{\tilde{\gamma}_{+}^{0, l}(N)}+f_{0}^{S,-}(N)\left(\frac{\alpha_{s}\left(Q_{I}^{2}\right)}{\alpha_{s}\left(Q^{2}\right)}\right)^{\tilde{\gamma}_{-}^{0, l}(N)}, \\
& g_{0}\left(N, Q^{2}\right)=g_{0}^{+}(N)\left(\frac{\alpha_{s}\left(Q_{I}^{2}\right)}{\alpha_{s}\left(Q^{2}\right)}\right)^{\tilde{\gamma}_{+}^{0, l}(N)}+g_{0}^{-}(N)\left(\frac{\alpha_{s}\left(Q_{I}^{2}\right)}{\alpha_{s}\left(Q^{2}\right)}\right)^{\tilde{\gamma}_{-}^{0, l}(N)}
\end{aligned}
$$

where $\tilde{\gamma}_{+}^{0, l}(N)$ and $\tilde{\gamma}_{-}^{0, l}(N)$ are the eigenvalues of the zeroth-order matrix for the $\tilde{\gamma}$ 's, and $f_{0}^{S,+}(N)+$ $f_{0}^{S,-}(N)=f_{0}^{S}(N)$ and $g_{0}^{+}(N)+g_{0}^{-}(N)=g_{0}(N)$. As with the nonsinglet quark distributions, the lowest-order inputs for the partons are $Q_{I}^{2}$-independent. Also, if we expect any powerlike behaviour to come only from perturbative effects, then these $Q_{I}^{2}$-independent inputs for the quark and gluon are analytic for $N>0$.

We may also discuss the relationship to the solutions using the evolution equations for the singlet structure functions. Unlike the nonsinglet case it is rather difficult to see how to express the solution in terms of factorized inputs and evolution parts; the evolution parts will not be simple exponentials, as in (4.16). However, the solution for the structure functions can be written as a series of terms each of which can be factored into a part dependent only on $\alpha_{s}\left(Q_{I}^{2}\right)$ and one depending on both $\alpha_{s}\left(Q_{I}^{2}\right)$ and $\alpha_{s}\left(Q^{2}\right)$, the latter either vanishing at $Q^{2}=Q_{I}^{2}$, or being equal to $\left(\alpha_{s}\left(Q_{I}^{2}\right) / \alpha_{s}\left(Q^{2}\right)\right)^{\tilde{\gamma}_{+,-}^{0, l}(-1)}$ and thus equal to unity at $Q^{2}=Q_{I}^{2}$. For each of these terms the former part may be interpreted as an input and the latter part may be interpreted as the evolution. Within the loop expansion we then include all terms where the order of the input part plus the order of the evolution part sums to less than or equal to some integer $n$.

By examining the form of the inputs under a change in starting scale, as with the nonsinglet structure functions, we find that the only fundamentally nonperturbative parts of the inputs are the zeroth-order parts, $F_{2,0}^{S}(N)$ and $F_{L, 0}^{S}(N)$, with all other inputs being in principle expressed as perturbative functions of the physical anomalous dimensions multiplying one of these nonperturbative components. They are given in terms of the parton inputs by

$$
F_{2,0}^{S}(N)=f_{0}^{S}(N)
$$

and

$$
F_{L, 0}^{S}(N)=C_{L, 1}^{f}(N) f_{0}^{S}(N)+C_{L, 1}^{g}(N) g_{0}(N) .
$$

We choose to think of the expressions for the structure functions as the real nonperturbative functions, since they are, of course, the physically relevant quantities.

The extra complexity of the solution in the singlet case, when compared to the nonsinglet case, also means it is far from obvious how to express the physical anomalous dimensions in terms 
of the parton anomalous dimensions and coefficient functions simply by comparing the forms of the solutions in the partonic language and purely in terms of structure functions. However, the expressions for the physical anomalous dimensions can be found at each order using (3.5). One could then solve for the structure functions in a manner different from the loop expansion, e.g. calculate the solution to the whole evolution equation for the structure functions using the physical anomalous dimensions up to a given order. This would be by definition factorization scheme invariant, but would only be renormalization scheme invariant up to the same order in the solution as the order of the anomalous dimensions. The rest of the solution would contain only a subset of the possible terms of a given form obtained from the full calculation. This subset will not necessarily be a good representation of the full set of terms obtained at this order, and would therefore have no real significance, and should be dropped. Hence, the evolution equations in terms do nothing to alter the strict ordering of the solution using this method of expansion.

This whole discussion of the order-by-order-in- $\alpha_{s}$ expansion scheme is perhaps a little academic since the errors invoked in performing the calculations without paying strict heed to the formally correct procedures are rather small. For example, when using this standard method of expansion only two different factorization schemes are generally considered, the $\overline{\mathrm{MS}}$ scheme and the DIS scheme; the latter related to the former by a change of parton distributions, so that the singlet quark distribution is equal to the singlet structure function. If a calculation is made in one scheme to a well-defined order, and then a transformation to the other scheme made correctly, precisely the same result will be obtained. In practice, small differences are noticed between calculations using different schemes within the loop expansion to NLO, but these come from well-understood sources. One common source is that the starting distributions in both schemes are described by a simple functional form, e.g.

$$
x f\left(x, Q_{I}^{2}\right)=A\left(Q_{I}^{2}\right) x^{-\lambda\left(Q_{I}^{2}\right)}(1-x)^{\eta\left(Q_{I}^{2}\right)}\left(1+\epsilon\left(Q_{I}^{2}\right) x^{1 / 2}+\gamma\left(Q_{I}^{2}\right) x\right),
$$

rather than the formally correct expression of a power series in $\alpha_{s}\left(Q_{I}^{2}\right)$ with essentially perturbative coefficients convoluted with a $Q_{I}^{2}$-independent nonperturbative function of $x$. If the starting distribution is of the form above in one scheme, then in the other scheme it will not be modelled precisely by a function of the same form. However, the error is in general small. Alternatively, if the calculations are not done in a well-ordered manner, then differences between the calculation done in the two schemes, or between this type of calculation and the correct NLO calculation, will be of NNLO. Again this usually results in only small differences. ${ }^{9}$ Similarly, small differences would be obtained by working in two different renormalization schemes.

\footnotetext{
${ }^{9}$ For a comparison of calculations done at NLO using different methods see [35].
} 
Nevertheless, there are a couple of points we wish to make here, concerning the loop expansion. Firstly we note that if one considers the input to be consistent with the loop expansion, e.g. NLOorder evolution should be accompanied by a NLO input, the power of $\lambda\left(Q_{I}^{2}\right)$ should not correspond to parton distributions much steeper than flat for the singlet quark or gluon at this order. This is because the first-order-in- $\alpha_{s}\left(Q_{I}^{2}\right)$ input should be accompanied by no more than a single power of $\ln (1 / x)$. Restricting $\lambda\left(Q_{I}^{2}\right)$ in this way is rather important for the fits to the low $x$ data, and would mean that NLO fits to small- $x$ data would be very poor. The only way to avoid this is to let $\lambda\left(Q_{I}^{2}\right)$ be an artificial free parameter, in which case, if (4.24) describes the singlet quark density, it must be $\sim 0.2-0.3$ for practically any $Q_{I}^{2}$. This value is totally unjustified, and the need for this steepness in the input for the quark is a clear sign of the limited usefulness of the NLO-in- $\alpha_{s}$ calculation at small $x$.

There is another reason for being concerned about the validity of the loop expansion at small $x$. The reason for the relative smallness of the differences between inaccurately performed NLO calculations noted above, even at small $x$, is that the differences between these calculations do not contain terms which are any more leading in $\ln (1 / x)$ than the NLO calculation itself. Hence they are genuinely an order of $\alpha_{s}$ down on the NLO calculation, with no small- $x$ enhancement. This is also true for calculations done in different renormalization schemes (or at different renormalization scales). However, the real NNLO contribution are higher order in $\alpha_{s}$ but also contain terms at higher order in $\ln (1 / x)$, and are potentially large at small $x$. Hence, the relative insensitivity of structure functions to changes in renormalization or factorization scheme for calculations which are not carefully ordered is no guarantee that genuine higher-order corrections will be small at small $x$ when using the loop expansion. Indeed we would naively expect them to be large.

In contrast to the insensitivity when using the loop expansion, when using the leading- $\ln (1 / x)$ expansion very large differences between calculations done in a large number of different factorization schemes have been noted. This is an clear sign that the calculations are not being done in a well-ordered manner, and that the ambiguity introduced by lack of care in the calculations is greater in this method of expansion than the standard loop expansion. We will now demonstrate that this is indeed the case.

\subsection{The Leading $-\ln (1 / x)$ Expansion.}

It should clearly be possible to define a well-ordered expansion in leading powers of $\ln (1 / x)$, or equivalently, in leading powers of $1 / N$ in moment space. We will now demonstrate that this is indeed the case. As in the loop expansion, we will first work in terms of the traditional parton distribution functions and coefficient functions, and see how this results in expressions containing the physical anomalous dimensions. Doing this enables us to see how large factorization scheme dependence can arise when calculating less carefully within this expansion scheme. 
First we must set up our notation. In this expansion scheme we write,

$$
\tilde{\gamma}\left(N, Q^{2}\right)=\sum_{n=0}^{\infty} \alpha_{s}^{n}\left(Q^{2}\right) \sum_{m=1-n}^{\infty} \tilde{\gamma}^{n m} \alpha_{s}^{m}\left(Q^{2}\right) N^{-m} \equiv \sum_{n=0}^{\infty} \alpha_{s}^{n}\left(Q^{2}\right) \tilde{\gamma}^{n}\left(\alpha_{s}\left(Q^{2}\right) / N\right) .
$$

In particular, in the $\overline{\mathrm{MS}}$ renormalization and factorization scheme we may write

$$
\tilde{\gamma}_{g g}\left(N, \alpha_{s}\left(Q^{2}\right)\right)=\sum_{n=0}^{\infty} \alpha_{s}^{n}\left(Q^{2}\right) \tilde{\gamma}_{g g}^{n}\left(\alpha_{s}\left(Q^{2}\right) / N\right)
$$

where the series expansion for $\tilde{\gamma}_{g g}^{0}\left(\alpha_{s} / N\right)$ is known to all orders (all the coefficients being positive). This expression for $\tilde{\gamma}_{g g}^{0}\left(\alpha_{s} / N\right)$ is renormalization scheme independent. $\tilde{\gamma}_{g g}^{1}\left(\alpha_{s} / N\right)$ is of course renormalization scheme dependent, since it must change to absorb part of the effect of the $\mathcal{O}\left(\alpha_{s}^{2}\right)$ change in the coupling on $\tilde{\gamma}_{g g}^{0}\left(\alpha_{s} / N\right)$. The renormalization scheme independence is also true for $\tilde{\gamma}_{g f}^{0}\left(\alpha_{s} / N\right)$, which obeys (1.1), and also for $\tilde{\gamma}_{f f}^{0}\left(\alpha_{s} / N\right)$. There is also a renormalization-schemeindependent relationship between $\tilde{\gamma}_{f f}^{1}\left(\alpha_{s} / N\right)$ and $\tilde{\gamma}_{f g}^{1}\left(\alpha_{s} / N\right)$, which tells us that

$$
\tilde{\gamma}_{f f}^{1}\left(\alpha_{s} / N\right)=\frac{4}{9}\left(\tilde{\gamma}_{f g}^{1}\left(\alpha_{s} / N\right)-\frac{2 N_{f}}{6 \pi b_{0}}\right)
$$

where the second term in the brackets is the one-loop contribution to $\tilde{\gamma}_{f g}^{1}\left(\alpha_{s} / N\right)$. It is also known that neither the nonsinglet anomalous dimension nor the nonsinglet coefficient function have poles at $N=0$. Hence, the nonsinglet sector makes very little contribution to the structure function at small $x$, and as such we will ignore it for the remainder of this section.

A general change in factorization scheme may be expressed by writing an element of the transformation matrix $U$ as

$$
U_{a b}\left(N, \alpha_{s}\left(Q^{2}\right)\right)=\sum_{n=0}^{\infty} \alpha_{s}^{n}\left(Q^{2}\right) \sum_{m=1-n}^{\infty} U_{a b}^{n m} \alpha_{s}^{m}\left(Q^{2}\right) N^{-m} \equiv \sum_{n=0}^{\infty} \alpha_{s}^{n}\left(Q^{2}\right) U_{a b}^{n}\left(\alpha_{s}\left(Q^{2}\right) / N\right),
$$

with condition on the $U_{a b}^{n m}$ such that $U$ obeys $U_{a b}=\delta_{a b}+\mathcal{O}\left(\alpha_{s}\right)$. A change of factorization scheme with $U_{a b}^{0} \neq 0$ can introduce scheme dependence into the $\tilde{\gamma}_{a b}^{0}\left(\alpha_{s} / N\right)$ 's, as we see from (2.14). Insisting that $\tilde{\gamma}_{g g}^{0}\left(\alpha_{s} / N\right), \tilde{\gamma}_{g f}^{0}\left(\alpha_{s} / N\right), \tilde{\gamma}_{f f}^{0}\left(\alpha_{s} / N\right)$ and $\tilde{\gamma}_{f g}^{0}\left(\alpha_{s} / N\right)$ are unaltered by scheme changes leads to the requirement,

$$
\begin{array}{lrl}
U_{f g}^{0}\left(N, \alpha_{s}\right) & =0, & U_{f f}^{0}\left(N, \alpha_{s}\right)=1, \\
U_{g g}^{0}\left(\alpha_{s} / N\right) & =1+\sum_{n=1}^{\infty} U_{g g}^{0, n}\left(\alpha_{s}\left(Q^{2}\right) / N\right)^{n}, & U_{g f}^{0}\left(\alpha_{s} / N\right)=\frac{4}{9}\left(U_{g g}^{0}\left(\alpha_{s} / N\right)-1\right) .
\end{array}
$$

This requirement also preserves the relationship (4.27) between $\tilde{\gamma}_{f f}^{1}\left(\alpha_{s} / N\right)$ and $\tilde{\gamma}_{f g}^{1}\left(\alpha_{s} / N\right)$. For simplicity, and due to factorization scheme invariance of physical quantities, we will only consider factorization scheme changes away from $\overline{\mathrm{MS}}$ scheme of the type (4.29). The $U_{a b}^{n}\left(\alpha_{s} / N\right)$ for $n>0$ will have no restrictions. 
Restricting ourselves to these schemes we may write

$$
C_{2}^{S}\left(N, \alpha_{s}\right)=1+\sum_{n=1}^{\infty} \alpha_{s}^{n}\left(Q^{2}\right) \sum_{m=1-n}^{\infty} C_{2, n, m}^{S} \alpha_{s}^{m}\left(Q^{2}\right) N^{-m} \equiv 1+\sum_{n=1}^{\infty} \alpha_{s}^{n}\left(Q^{2}\right) C_{2, n}^{S}\left(\alpha_{s} / N\right),
$$

and all other coefficient functions as

$$
C_{i}^{a}\left(N, \alpha_{s}\right)=\sum_{n=1}^{\infty} \alpha_{s}^{n}\left(Q^{2}\right) \sum_{m=1-n}^{\infty} C_{i, n, m}^{a} \alpha_{s}^{m}\left(Q^{2}\right) N^{-m} \equiv \sum_{n=1}^{\infty} \alpha_{s}^{n}\left(Q^{2}\right) C_{i, n}^{a}\left(\alpha_{s} / N\right) .
$$

All the $C_{i, n}^{a}\left(\alpha_{s} / N\right)$ are both renormalization-scheme- and factorization-scheme-dependent quantities. Indeed, all of the $C_{i, n, m}^{a}$ are renormalization scheme and factorization scheme dependent, except for the $C_{L, n, 1-n}^{a}$, which come from the one-loop longitudinal coefficient functions which, as we saw in the previous subsection, are totally scheme independent. There are also two renormalization and factorization scheme (with our restrictions) independent relationships between coefficient functions:

$$
C_{2,1}^{S}\left(\alpha_{s} / N\right)=\frac{4}{9}\left(C_{2,1}^{g}\left(\alpha_{s} / N\right)-C_{2,1,0}^{g}\right),
$$

where the second term in brackets is the one-loop contribution to $C_{2,1}^{g}\left(\alpha_{s} / N\right)$, which is itself renormalization scheme and factorization scheme dependent (being equal to $\left(N_{f} / 6 \pi\right)$ in $\overline{\mathrm{MS}}$ scheme); and

$$
\left(C_{L, 1}^{S}\left(\alpha_{s} / N\right)-\frac{2}{3 \pi}\right)=\frac{4}{9}\left(C_{2,1}^{g}\left(\alpha_{s} / N\right)-\frac{2 N_{f}}{6 \pi}\right)
$$

where the second terms in the brackets are the one-loop contributions to $C_{L, 1}^{S}\left(\alpha_{s} / N\right)$ and $C_{L, 1}^{g}\left(\alpha_{s} / N\right)$, both of which are renormalization and factorization scheme independent.

Working in an arbitrary factorization scheme (up to the above restrictions) and using the general expressions for the $\tilde{\gamma}$ 's and coefficient functions, we may find expressions for the structure functions. The first step towards this is solving the renormalization group equations for the parton distributions. The lowest-order part of the equation is,

$$
\alpha_{s}^{2}\left(Q^{2}\right) \frac{d}{d \alpha_{s}\left(Q^{2}\right)}\left(\begin{array}{c}
f_{0}^{S}\left(N, Q^{2}\right) \\
g_{0}\left(N, Q^{2}\right)
\end{array}\right)=-\left(\begin{array}{cc}
0 & 0 \\
\frac{4}{9} \tilde{\gamma}_{g g}^{0}\left(\alpha_{s} / N\right) & \tilde{\gamma}_{g g}^{0}\left(\alpha_{s} / N\right)
\end{array}\right)\left(\begin{array}{c}
f_{0}^{S}\left(N, Q^{2}\right) \\
g_{0}\left(N, Q^{2}\right)
\end{array}\right)
$$

This may easily be solved to give

$$
\begin{aligned}
& f_{0}^{S}\left(N, Q^{2}\right)=f_{0}^{S}\left(N, Q_{I}^{2}\right), \\
& g_{0}\left(N, Q^{2}\right)=\left(g_{0}\left(N, Q_{I}^{2}\right)+\frac{4}{9} f_{0}^{S}\left(N, Q_{I}^{2}\right)\right) \exp \left[\int_{\alpha_{s}\left(Q^{2}\right)}^{\alpha_{s}\left(Q_{I}^{2}\right)} \frac{\tilde{\gamma}_{g g}^{0}\left(\alpha_{s}\left(q^{2}\right) / N\right)}{\alpha_{s}^{2}\left(q^{2}\right)} d \alpha_{s}\left(q^{2}\right)\right]-\frac{4}{9} f_{0}^{S}\left(N, Q_{I}^{2}\right) .
\end{aligned}
$$

This is analogous to the lowest-order solution within the loop expansion and contains two factors, one of which must appear in all the higher terms in the expansion: instead of $\left(\alpha_{s}\left(Q_{I}^{2}\right) / \alpha_{s}\left(Q^{2}\right)\right)^{\tilde{\gamma}_{+,-}^{0, l}(N)}$, corresponding to the two eigenvalues of $\tilde{\gamma}^{0, l}(N)$ in the loop expansion, we 
have $\exp \left[\int_{\alpha_{s}\left(Q^{2}\right)}^{\alpha_{s}\left(Q_{I}^{2}\right)} \frac{\tilde{\gamma}_{g g}^{0}\left(\alpha_{s}\left(q^{2}\right) / N\right)}{\alpha_{s}^{2}\left(q^{2}\right)} d \alpha_{s}\left(q^{2}\right)\right]$ and 1, corresponding to the two eigenvalues of $\tilde{\gamma}^{0}\left(\alpha_{s} / N\right)$ in the leading $-\ln (1 / x)$ expansion, i.e. $\tilde{\gamma}_{g g}^{0}\left(\alpha_{s} / N\right)$ and 0.

The fact that the leading order part of $\tilde{\gamma}_{g g}$ and $\tilde{\gamma}_{g f}$ is more leading than the leading part of $\tilde{\gamma}_{f g}$ and $\tilde{\gamma}_{f f}$, in this expansion scheme makes obtaining a well-ordered solution rather more complicated than in the loop-expansion. (The same is true when using the physical anomalous dimensions since $\tilde{\Gamma}_{2 L}$ and $\tilde{\Gamma}_{22}$ both equal 0.) Hence, before using our solutions for the parton densities to construct expressions for the structure functions we will need to solve higher-order renormalization group equations in order to determine the general form of the solutions. This is done iteratively using evolution equation ordered analogously to (4.14), and in order to get useful solutions we must go out to $n=\infty$, keeping only the most leading terms each time we iterate. With some work and a straightforward inductive proof we obtain

$$
\begin{aligned}
f^{S}\left(N, Q^{2}\right)= & \alpha_{s}\left(Q^{2}\right) \frac{\tilde{\gamma}_{f g}^{1}\left(\alpha_{s}\left(Q^{2}\right) / N\right)}{\tilde{\gamma}_{g g}^{0}\left(\alpha_{s}\left(Q^{2}\right) / N\right)}\left(g_{0}(N)+\frac{4}{9} f_{0}^{S}(N)+\tilde{g}_{0}\left(N, Q_{I}^{2}\right)\right) \times \\
& \exp \left[\int_{\alpha_{s}\left(Q^{2}\right)}^{\alpha_{s}\left(Q_{I}^{2}\right)} \frac{\tilde{\gamma}_{g g}^{0}\left(\alpha_{s}\left(q^{2}\right) / N\right)}{\alpha_{s}^{2}\left(q^{2}\right)}+\frac{\tilde{\gamma}_{g g}^{1}\left(\alpha_{s}\left(q^{2}\right) / N\right)+\frac{4}{9} \tilde{\gamma}_{f g}^{1}\left(\alpha_{s}\left(q^{2}\right) / N\right)}{\alpha_{s}\left(q^{2}\right)} d \alpha_{s}\left(q^{2}\right)\right] \\
& +f_{0}^{S}(N)\left(\frac{\alpha_{s}\left(Q_{I}^{2}\right)}{\alpha_{s}\left(Q^{2}\right)}\right)^{-\frac{4}{9} \frac{2 N_{f}}{6 \pi b_{0}}}+\tilde{f}_{1}^{S}\left(N, Q_{I}^{2}\right)+\text { higher order in } \alpha_{s} \text { and /or } N,
\end{aligned}
$$

and,

$$
\begin{aligned}
g\left(N, Q^{2}\right)= & \left(g_{0}(N)+\frac{4}{9} f_{0}^{S}(N)+\tilde{g}_{0}\left(N, Q_{I}^{2}\right)\right) \times \\
& \quad \exp \left[\int_{\alpha_{s}\left(Q^{2}\right)}^{\alpha_{s}\left(Q_{I}^{2}\right) \tilde{\gamma}_{g g}^{0}\left(\alpha_{s}\left(q^{2}\right) / N\right)} \frac{\tilde{\gamma}_{g g}^{1}\left(\alpha_{s}\left(q^{2}\right) / N\right)+\frac{4}{9} \tilde{\gamma}_{f g}^{1}\left(\alpha_{s}\left(q^{2}\right) / N\right)}{\alpha_{s}^{2}\left(q^{2}\right)} d \alpha_{s}\left(q^{2}\right)\right] \\
& -\frac{4}{9} f_{0}^{S}(N)\left(\frac{\alpha_{s}\left(Q_{I}^{2}\right)}{\alpha_{s}\left(Q^{2}\right)}\right)^{-\frac{4}{9} \frac{2 N_{f}}{6 \pi b_{0}}}+\text { higher order in } \alpha_{s} \text { and/or } N .
\end{aligned}
$$

We may also write the expression for the rate of change of the quark distribution

$$
\begin{aligned}
-\alpha_{s}^{2}\left(Q^{2}\right) \frac{d f^{S}\left(N, Q^{2}\right)}{d \alpha_{s}\left(Q^{2}\right)}= & \alpha_{s}\left(Q^{2}\right) \tilde{\gamma}_{f g}^{1}\left(\alpha_{s}\left(Q^{2}\right) / N\right)\left(g_{0}(N)+\frac{4}{9} f_{0}^{S}(N)+\tilde{g}_{0}\left(N, Q_{I}^{2}\right)\right) \times \\
& \exp \left[\int_{\alpha_{s}\left(Q^{2}\right)}^{\alpha_{s}\left(Q_{I}^{2}\right)} \frac{\tilde{\gamma}_{g g}^{0}\left(\alpha_{s}\left(q^{2}\right) / N\right)}{\alpha_{s}^{2}\left(q^{2}\right)}+\frac{\tilde{\gamma}_{g g}^{1}\left(\alpha_{s}\left(q^{2}\right) / N\right)+\frac{4}{9} \tilde{\gamma}_{f g}^{1}\left(\alpha_{s}\left(q^{2}\right) / N\right)}{\alpha_{s}\left(q^{2}\right)} d \alpha_{s}\left(q^{2}\right)\right] \\
& -\frac{4}{9} \alpha_{s}\left(Q^{2}\right) f_{0}^{S}(N) \frac{2 N_{f}}{6 \pi b_{0}}\left(\frac{\alpha_{s}\left(Q_{I}^{2}\right)}{\alpha_{s}\left(Q^{2}\right)}\right)^{-\frac{4}{9} \frac{2 N_{f}}{6 \pi b_{0}}}+\text { higher order in } \alpha_{s} \text { and/or } N .
\end{aligned}
$$

This last expression will be important since at leading order $\alpha_{s}^{2}\left(Q^{2}\right)\left(d F_{2}\left(N, Q^{2}\right) / d \alpha_{s}\left(Q^{2}\right)\right)$ is directly related to $d F_{2}\left(N, Q^{2}\right) / d \ln Q^{2}$ which we will wish to study as well as $F_{2}\left(N, Q^{2}\right)$. 
The input $g_{0}\left(N, Q_{I}^{2}\right)$ is chosen so that the change in $\exp \left[\int_{\alpha_{s}\left(Q^{2}\right)}^{\alpha_{s}\left(Q_{g}^{2}\right)} \frac{\tilde{\gamma}_{g g}^{0}\left(\alpha_{s}\left(q^{2}\right) / N\right)}{\alpha_{s}^{2}\left(q^{2}\right)} d \alpha_{s}\left(q^{2}\right)\right]$ under a change in $Q_{I}^{2}$ can be compensated for by a change in $g_{0}\left(N, Q_{I}^{2}\right)$ up to corrections of higher order. Hence, the gluon input may be written as

$$
\begin{aligned}
& \left(g_{0}(N)+\frac{4}{9} f_{0}^{S}(N)+\tilde{g}_{0}\left(N, Q_{I}^{2}\right)\right) \\
& \quad=\left(g_{0}(N)+\frac{4}{9} f_{0}^{S}(N)\right)\left(1+\sum_{m=1}^{\infty} \tilde{g}_{0, m}\left(\frac{\alpha_{s}\left(Q_{I}^{2}\right)}{N}\right)^{m}\right) \exp \left[\ln \left(Q_{I}^{2} / A_{g g}\right) \gamma_{g g}^{0}\left(\alpha_{s}\left(Q_{I}^{2}\right) / N\right)\right],
\end{aligned}
$$

where $A_{g g}$ is an unknown scale. The series $\sum_{m=1}^{\infty} \tilde{g}_{0, m}\left(\alpha_{s}\left(Q_{I}^{2}\right) / N\right)^{m}$ is at yet undetermined, but is potentially renormalization and factorization scheme dependent. It will only be determined when we come to construct the structure functions themselves. In the same manner we can determine the general form of our input, $f_{1}^{S}\left(N, Q_{I}^{2}\right)$, obtaining

$$
f_{1}^{S}\left(N, Q_{I}^{2}\right)=\left(g_{0}(N)+\frac{4}{9} f_{0}^{S}(N)+\tilde{g}_{0}\left(N, Q_{I}^{2}\right)\right) \alpha_{s}\left(Q_{I}^{2}\right) \frac{\gamma_{f g}^{1}\left(\alpha_{s}\left(Q_{I}^{2}\right) / N\right)}{\gamma_{g g}^{0}\left(\alpha_{s}\left(Q_{I}^{2}\right) / N\right)}+\tilde{f}_{1}^{S}\left(N, Q_{I}^{2}\right),
$$

where

$$
\tilde{f}_{1}^{S}\left(N, Q_{I}^{2}\right)=N \sum_{n=0}^{\infty} \tilde{f}_{1, m}^{S}\left(\frac{\alpha_{s}\left(Q_{I}^{2}\right)}{N}\right)^{m}\left(g_{0}(N)+\frac{4}{9} f_{0}^{S}(N)\right)
$$

is potentially renormalization and factorization scheme dependent, but not yet determined.

We also consider the form of the $N$-dependence of our inputs. If we take the point of view that any steep behaviour in the parton distributions only comes about due to perturbative effects, then we assume that $f_{0}^{S}(N)$ and $g_{0}(N)$ are both soft, i.e. either flat or even valence-like when the transform to $x$-space is performed (or at most going like a finite, small power of $\ln (1 / x)$ ). This requires that they both be analytic for $N>0$. As in the loop expansion, we may think of $g_{0}(N)$, and $f_{0}^{S}(N)$ as fundamentally soft, nonperturbative parts of the input. ${ }^{10}$ The parts multiplying these are then really determined by perturbation theory. Since we are meant to be expanding our solution for the structure functions in powers of both $\alpha_{s}$ and $N$, it might be argued that we should expand $f_{0}^{S}(N)$ and $g_{0}(N)$ in powers of $N$. We feel this is not really appropriate since it is the perturbative part of the solution for which we are able to solve, and thus which we are able to order correctly, and in the expressions for the structure functions the whole of the nonperturbative inputs should multiply the well-ordered perturbative parts of the solution.

It is now possible to examine the form of the solutions for the structure functions. We can construct the leading part of the full solutions by combining our solutions for the parton distributions

10 Of course, we should be trying to find fundamentally nonperturbative inputs for the structure functions rather than the partons, as discussed for the loop expansion. However, in this expansion scheme $f_{0}^{S}(N)$ and $g_{0}(N)$ are trivially related to $F_{2,0}(N)$ and $\hat{F}_{L, 0}(N)$ as we will see soon. 
with the zeroth- and first-order coefficient functions. This gives

$$
\begin{aligned}
F_{L}\left(N, Q^{2}\right)= & \alpha_{s}\left(Q^{2}\right) C_{L, 1}^{g}\left(\alpha_{s}\left(Q^{2}\right) / N\right)\left(g_{0}(N)+\frac{4}{9} f_{0}^{S}(N)+\tilde{g}_{0}\left(N, Q_{I}^{2}\right)\right) \times \\
& \quad \exp \left[\int_{\alpha_{s}\left(Q^{2}\right)}^{\alpha_{s}\left(Q_{I}^{2}\right)} \frac{\tilde{\gamma}_{g g}^{0}\left(\alpha_{s}\left(q^{2}\right) / N\right)}{\alpha_{s}^{2}\left(q^{2}\right)}+\frac{\tilde{\gamma}_{g g}^{1}\left(\alpha_{s}\left(q^{2}\right) / N\right)+\frac{4}{9} \tilde{\gamma}_{f g}^{1}\left(\alpha_{s}\left(q^{2}\right) / N\right)}{\alpha_{s}\left(q^{2}\right)} d \alpha_{s}\left(q^{2}\right)\right] \\
+ & \alpha_{s}\left(Q^{2}\right)\left(C_{L, 1,0}^{S}-\frac{4}{9} C_{L, 1,0}^{g}\right) f_{0}^{S}(N)\left(\frac{\alpha_{s}\left(Q_{I}^{2}\right)}{\alpha_{s}\left(Q^{2}\right)}\right)^{-\frac{4}{9} \frac{2 N_{f}}{6 \pi b_{0}}}+\text { higher order in } \alpha_{s} \text { and/or } N,
\end{aligned}
$$

where (4.33) has been used. Also

$$
\begin{aligned}
F_{2}\left(N, Q^{2}\right) & \left.=\alpha_{s}\left(Q^{2}\right) \frac{\tilde{\gamma}_{f g}^{1}\left(\alpha_{s}\left(Q^{2}\right) / N\right)}{\tilde{\gamma}_{g g}^{0}\left(\alpha_{s}\left(Q^{2}\right) / N\right)}+C_{2,1}^{g}\left(\alpha_{s}\left(Q^{2}\right) / N\right)\right)\left(g_{0}(N)+\frac{4}{9} f_{0}^{S}(N)+\tilde{g}_{0}\left(N, Q_{I}^{2}\right)\right) \times \\
& \exp \left[\int_{\alpha_{s}\left(Q^{2}\right)}^{\alpha_{s}\left(Q_{I}^{2}\right)} \frac{\tilde{\gamma}_{g g}^{0}\left(\alpha_{s}\left(q^{2}\right) / N\right)}{\alpha_{s}^{2}\left(q^{2}\right)}+\frac{\tilde{\gamma}_{g g}^{1}\left(\alpha_{s}\left(q^{2}\right) / N\right)+\frac{4}{9} \tilde{\gamma}_{f g}^{1}\left(\alpha_{s}\left(q^{2}\right) / N\right)}{\alpha_{s}\left(q^{2}\right)} d \alpha_{s}\left(q^{2}\right)\right] \\
& +f_{0}^{S}(N)\left(\frac{\alpha_{s}\left(Q_{I}^{2}\right)}{\alpha_{s}\left(Q^{2}\right)}\right)^{-\frac{4}{9} \frac{2 N_{f}}{6 \pi b_{0}}}+\tilde{f}_{1}^{S}\left(N, Q_{I}^{2}\right)+\text { higher order in } \alpha_{s} \text { and/or } N,
\end{aligned}
$$

and

$$
\begin{aligned}
& -\alpha_{s}^{2}\left(Q^{2}\right) \frac{d F_{2}\left(N, Q^{2}\right)}{d \alpha_{s}\left(Q^{2}\right)}=\left(\alpha_{s}\left(Q^{2}\right) \tilde{\gamma}_{f g}^{1}\left(\alpha_{s}\left(Q^{2}\right) / N\right)+\right. \\
& \left.\alpha_{s}\left(Q^{2}\right) C_{2,1}^{g}\left(\alpha_{s}\left(Q^{2}\right) / N\right) \tilde{\gamma}_{g g}^{0}\left(\alpha_{s}\left(Q^{2}\right) / N\right)\right)\left(g_{0}(N)+\frac{4}{9} f_{0}^{S}(N)+\tilde{g}_{0}\left(N, Q_{I}^{2}\right)\right) \times \\
& \quad \exp \left[\int_{\alpha_{s}\left(Q^{2}\right)}^{\alpha_{s}\left(Q_{I}^{2}\right)} \frac{\tilde{\gamma}_{g g}^{0}\left(\alpha_{s}\left(q^{2}\right) / N\right)}{\alpha_{s}^{2}\left(q^{2}\right)}+\frac{\tilde{\gamma}_{g g}^{1}\left(\alpha_{s}\left(q^{2}\right) / N\right)+\frac{4}{9} \tilde{\gamma}_{f g}^{1}\left(\alpha_{s}\left(q^{2}\right) / N\right)}{\alpha_{s}\left(q^{2}\right)} d \alpha_{s}\left(q^{2}\right)\right] \\
& -\alpha_{s}\left(Q^{2}\right) \frac{4}{9} \frac{2 N_{f}}{6 \pi b_{0}} f_{0}^{S}(N)\left(\frac{\alpha_{s}\left(Q_{I}^{2}\right)}{\alpha_{s}\left(Q^{2}\right)}\right)^{-\frac{4}{9} \frac{2 N_{f}}{6 \pi b_{0}}}+\text { higher order in } \alpha_{s} \text { and/or } N,
\end{aligned}
$$

where, we drop the superscript $S$ for the structure functions for the rest of this subsection.

Each of these expressions will be factorization scheme independent since each represents the expression for a physical quantity up to corrections of a form different from the terms explicitly appearing and which we have deemed to be higher order in our expansion scheme. However, they are not in themselves the leading order expressions. In order to derive these we begin with the longitudinal structure function. Making the definition

$\Phi_{1}^{+}\left(Q^{2}, Q_{I}^{2}\right)=\int_{\alpha_{s}\left(Q^{2}\right)}^{\alpha_{s}\left(Q_{I}^{2}\right)} \frac{\tilde{\gamma}_{g g}^{1}\left(\alpha_{s}\left(q^{2}\right) / N\right)+\frac{4}{9} \tilde{\gamma}_{f g}^{1}\left(\alpha_{s}\left(q^{2}\right) / N\right)}{\alpha_{s}\left(q^{2}\right)}-\frac{d}{d \alpha_{s}\left(q^{2}\right)}\left(\ln \left(\frac{C_{L, 1}^{g}\left(\alpha_{s}\left(q^{2}\right) / N\right)}{C_{L, 1,0}^{g}}\right)\right) d \alpha_{s}\left(q^{2}\right)$,

where $\Phi_{1}^{+}\left(Q^{2}, Q_{I}^{2}\right)$ is factorization-scheme independent (as can be checked using the rules (2.12)(2.14)) and $\Phi_{1}^{+}\left(Q_{I}^{2}, Q_{I}^{2}\right)=1$, we may factorize (4.42) completely into input and evolution parts, 
i.e.

$$
\begin{aligned}
F_{L}\left(N, Q^{2}\right) & =\alpha_{s}\left(Q_{I}^{2}\right) \frac{2 N_{f}}{6 \pi}\left(g_{0}(N)+\frac{4}{9} f_{0}^{S}(N)\right) \exp \left[\int_{\alpha_{s}\left(Q^{2}\right)}^{\alpha_{s}\left(Q_{I}^{2}\right)} \frac{\tilde{\gamma}_{g g}^{0}\left(\alpha_{s}\left(q^{2}\right) / N\right)}{\alpha_{s}^{2}\left(q^{2}\right)} d \alpha_{s}\left(q^{2}\right)\right] \times \\
& \left(\frac{C_{L, 1}^{g}\left(\alpha_{s}\left(Q_{I}^{2}\right) / N\right)}{C_{L, 1,0}^{g}} \cdot\left(1+\frac{\tilde{g}_{0}\left(N, Q_{I}^{2}\right)}{g_{0}(N)+\frac{4}{9} f_{0}^{S}(N)}\right)\right) \exp \left[\Phi_{1}^{+}\left(Q^{2}, Q_{I}^{2}\right)-\ln \left(\frac{\alpha_{s}\left(Q_{I}^{2}\right)}{\alpha_{s}\left(Q^{2}\right)}\right)\right] \\
& +\alpha_{s}\left(Q^{2}\right)\left(\frac{18-4 N_{f}}{27 \pi}\right) f_{0}^{S}(N)\left(\frac{\alpha_{s}\left(Q_{I}^{2}\right)}{\alpha_{s}\left(Q_{I}^{2}\right)}\right)^{-\frac{4}{9} \frac{2 N_{f}}{6 \pi b_{0}}-1}+\text { higher order in } \alpha_{s} \text { and/or } N .
\end{aligned}
$$

It is now possible to attach direct physical significance to each of the factorization-schemeindependent pieces appearing in this expression.

We first consider the inputs. Going to (4.43) for the moment we see that $f_{0}^{S}(N)$ is the only term in $F_{2}\left(N, Q_{I}^{2}\right)$ which is zeroth-order in our expansion scheme. As such it is the zeroth order input for $F_{2}\left(N, Q^{2}\right)$, and we may write

$$
f_{0}^{S}(N)=F_{2,0}(N),
$$

and this is one of our two fundamentally nonperturbative inputs. Also, from (4.46) we see that the total $\alpha_{s}\left(Q_{I}^{2}\right)$-independent input for $F_{L}\left(N, Q_{I}^{2}\right)$ (once we have divided out a single power of $\left.\alpha_{s}\left(Q_{I}^{2}\right) /(2 \pi)\right)$ is

$$
\frac{2 N_{f}}{3}\left(g_{0}(N)+\frac{2}{N_{f}} f_{0}^{S}(N)\right)=\hat{F}_{L, 0}(N)
$$

This is therefore equal to our other fundamentally nonperturbative physical input We also make a similar definition for the part of the input for $\hat{F}_{L}\left(N, Q^{2}\right)$ which is of the form $\sum_{n=1}^{\infty} a_{n}\left(Q_{I}^{2}\right)\left(\alpha_{s}\left(Q_{I}\right) / N\right)^{n}$ multiplying $\left(g_{0}(N)+\frac{4}{9} f_{0}^{S}(N)\right)$, or more correctly multiplying $\left(\hat{F}_{L, 0}(N)-\right.$ $\left.\left(\left(36-8 N_{f}\right) / 27\right) F_{2,0}(N)\right)$, to complete our definition of the input in (4.46) and write

$$
\begin{aligned}
\frac{2 N_{f}}{3}\left(g_{0}(N)+\frac{4}{9} f_{0}^{S}(N)+\tilde{g}_{0}\left(N, Q_{I}^{2}\right)\right) & \left(\frac{C_{L, 1}^{g}\left(\alpha_{s}\left(Q_{I}^{2}\right) / N\right)}{C_{L, 1,0}^{g}}\right) \\
& =\hat{F}_{L, 0}(N)-\left(\frac{36-8 N_{f}}{27}\right) F_{2,0}(N)+\tilde{\hat{F}}_{L, 0}\left(N, Q_{I}^{2}\right) .
\end{aligned}
$$

This whole expression must be both factorization scheme and renormalization scheme independent, facts which reveal information about the form of the gluon input. $C_{L, 1}^{g}\left(N, \alpha_{s}\left(Q_{I}^{2}\right)\right)$ is both renormalization and factorization-scheme dependent, so the scheme dependence of $\tilde{g}_{0}\left(N, Q_{I}^{2}\right)$ must be precisely so as to cancel this out, Hence, there is no reason for $\tilde{\hat{F}}_{L, 0}\left(\alpha_{s}\left(Q_{I}^{2}\right) / N\right)$ to depend on the leading-order longitudinal gluon coefficient function at all, and indeed, a natural choice seems to be that $\left(1+\sum_{m=1}^{\infty} \tilde{g}_{0, m}\left(\alpha_{s}\left(Q_{I}^{2}\right) / N\right)^{m}\right)$ is chosen equal to $\left(C_{L, 1,0}^{g} / C_{L, 1}^{g}\left(N, \alpha_{s}\left(Q_{I}^{2}\right)\right)\right.$ ) (it is difficult to see what else it could be chosen equal to), and therefore

$$
\begin{aligned}
\hat{F}_{L, 0}(N)-\left(\frac{36-8 N_{f}}{27}\right) F_{2,0}(N)+\tilde{\hat{F}}_{L, 0}\left(N, Q_{I}^{2}\right) \\
\quad=\left(\hat{F}_{L, 0}(N)-\left(\frac{36-8 N_{f}}{27}\right) F_{2,0}(N)\right) \exp \left[\ln \left(Q_{I}^{2} / A_{L L}\right) \gamma_{g g}^{0}\left(\alpha_{s}\left(Q_{I}^{2}\right) / N\right)\right] .
\end{aligned}
$$


Hence, we have a prediction for the input for the longitudinal structure function at small $x$ in terms of the nonperturbative inputs and some scale $A_{L L}$ (where $A_{L L}=A_{g g}$ from the previous subsection). As with $A_{N S}$ earlier, $A_{L L}$ is the scale at which the input is equal to the nonperturbative input alone, and hence we would expect it to be typical of the scale where perturbation theory starts to break down. $Q_{I}^{2}$ is a completely free parameter. We have constructed the solution to be formally insensitive to $Q_{I}^{2}$ at leading order, but there is clearly some residual $Q_{I}^{2}$-dependence. Hence, there will also be some optimum $Q_{I}^{2}$ to choose as the starting scale.

We may now examine the terms governing the evolution. $\exp \left[\int_{\alpha_{s}\left(Q^{2}\right)}^{\alpha_{s}\left(Q_{g g}^{2}\right)} \frac{\tilde{\gamma}_{g g}^{0}\left(\alpha_{s}\left(q^{2}\right) / N\right)}{\alpha_{s}^{2}\left(q^{2}\right)} d \alpha_{s}\left(q^{2}\right)\right]$ is the scheme-independent factor governing the small- $x$ growth with $Q^{2}$, and we make the definition

$$
\Phi_{0}^{+}\left(Q^{2}, Q_{I}^{2}\right)=\int_{\alpha_{s}\left(Q^{2}\right)}^{\alpha_{s}\left(Q_{I}^{2}\right)} \frac{\tilde{\gamma}_{g g}^{0}\left(\alpha_{s}\left(q^{2}\right) / N\right)}{\alpha_{s}^{2}\left(q^{2}\right)} d \alpha_{s}\left(q^{2}\right) .
$$

Of course, the other evolution factor resulting from the eigenvalues of the zeroth-order anomalous dimension was simply unity and as such $\Phi_{0}^{-}\left(Q^{2}, Q_{I}^{2}\right)$ does exist, but is implicitly zero. However, there is a correction to this factor of unity in (4.42)-(4.44), and we make the definition

$$
\Phi_{1}^{-}\left(Q^{2}, Q_{I}^{2}\right)=-\frac{4}{9} \frac{2 N_{f}}{6 \pi b_{0}} \ln \left(\frac{\alpha_{s}\left(Q_{I}^{2}\right)}{\alpha_{s}\left(Q^{2}\right)}\right) .
$$

Having made these factorization-scheme-invariant definitions for the inputs and evolution we may write the solution for $F_{L}\left(N, Q^{2}\right)$ as

$$
\begin{aligned}
F_{L}\left(N, Q^{2}\right) & =\frac{\alpha_{s}\left(Q_{I}^{2}\right)}{2 \pi}\left(\hat{F}_{L, 0}(N)+\tilde{\hat{F}}_{L, 0}\left(N, Q_{I}^{2}\right)-\left(\frac{36-8 N_{f}}{27}\right) F_{2,0}(N)\right) \times \\
& \exp \left[\Phi_{0}^{+}\left(Q^{2}, Q_{I}^{2}\right)+\Phi_{1}^{+}\left(Q^{2}, Q_{I}^{2}\right)-\ln \left(\frac{\alpha_{s}\left(Q_{I}^{2}\right)}{\alpha_{s}\left(Q^{2}\right)}\right)\right] \\
& +\frac{\alpha_{s}\left(Q_{I}^{2}\right)}{2 \pi}\left(\frac{36-8 N_{f}}{27}\right) F_{2,0}(N) \exp \left[\Phi_{1}^{-}\left(Q^{2}, Q_{I}^{2}\right)-\left(\ln \left(\frac{\alpha_{s}\left(Q_{I}^{2}\right)}{\alpha_{s}\left(Q^{2}\right)}\right)\right)\right] \\
& + \text { higher order in } \alpha_{s} \text { and/or } N .
\end{aligned}
$$

This is still of mixed order, but it is now relatively obvious how we may separate out the "leading part" from this expression. $\left[\Phi_{1}^{+}\left(Q^{2}, Q_{I}^{2}\right)-\ln \left(\alpha_{s}\left(Q_{I}^{2}\right) / \alpha_{s}\left(Q^{2}\right)\right)\right]$ contains the same type of terms as $\Phi_{0}^{+}\left(Q^{2}, Q_{I}^{2}\right)$, but each is a power of $N$ higher. Thus $\left[\Phi_{1}^{+}\left(Q^{2}, Q_{I}^{2}\right)-\ln \left(\alpha_{s}\left(Q_{I}^{2}\right) / \alpha_{s}\left(Q^{2}\right)\right)\right]$ is subleading to $\Phi_{0}^{+}\left(Q^{2}, Q_{I}^{2}\right)$, and indeed $\Phi_{1}^{+}\left(Q^{2}, Q_{I}^{2}\right)$ is a renormalization-scheme-dependent quantity, as it must be in order to absorb the change in $\Phi_{0}^{+}\left(Q^{2}, Q_{I}^{2}\right)$ resulting from a change in the definition of $\alpha_{s}\left(Q^{2}\right)$ under a change in renormalization scheme when working beyond leading order. Hence, we should factor $\exp \left[\Phi_{1}^{+}\left(Q^{2}, Q_{I}^{2}\right)-\ln \left(\alpha_{s}\left(Q_{I}^{2}\right) / \alpha_{s}\left(Q^{2}\right)\right)\right]$ out of the first term. Since $\left[\Phi_{1}^{-}\left(Q^{2}, Q_{I}^{2}\right)-\right.$ $\left.\ln \left(\alpha_{s}\left(Q_{I}^{2}\right) / \alpha_{s}\left(Q^{2}\right)\right)\right]$ is of the same form as the zeroth-order-in- $N$ part of $\Phi_{1}^{+}\left(Q^{2}, Q_{I}^{2}\right)$, then it should also be factored out of the leading-order expression for $F_{L}\left(N, Q^{2}\right)$. Thus, we are left with

$$
\begin{aligned}
F_{L}^{0}\left(N, Q^{2}\right)= & \frac{\alpha_{s}\left(Q_{I}^{2}\right)}{2 \pi}\left(\hat{F}_{L, 0}(N)+\tilde{\hat{F}}_{L, 0}\left(N, Q_{I}^{2}\right)-\left(\frac{36-8 N_{f}}{27}\right) F_{2,0}(N)\right) \exp \left[\Phi_{0}^{+}\left(Q^{2}, Q_{I}^{2}\right)\right] \\
& +\frac{\alpha_{s}\left(Q_{I}^{2}\right)}{2 \pi}\left(\frac{36-8 N_{f}}{27 \pi}\right) F_{2,0}(N) .
\end{aligned}
$$


Using the one-loop running coupling constant, as is appropriate for a leading-order expression, the whole of (4.54) is not only manifestly factorization scheme independent, but also renormalization scheme independent, as one would hope. Also, $\tilde{\hat{F}}_{L, 0}\left(N, Q_{I}^{2}\right)$ is constructed precisely so as to make the expression unchanged, at this order, under a change in starting scale. Hence, within this expansion scheme (4.54) is genuinely the leading-order expression for $F_{L}\left(N, Q^{2}\right)$.

We now turn our attention to the more phenomenologically important case of the structure function $F_{2}\left(N, Q^{2}\right)$. For technical simplicity we begin with the expression for $\alpha_{s}^{2}\left(Q^{2}\right)\left(d F_{2}\left(N, Q^{2}\right) / d \alpha_{s}\left(Q^{2}\right)\right)$. Using the definitions introduced in our discussion for the longitudinal structure function we may write (4.44) as

$$
\begin{gathered}
-\alpha_{s}^{2}\left(Q^{2}\right) \frac{d F_{2}\left(N, Q^{2}\right)}{d \alpha_{s}\left(Q^{2}\right)}=\left(\alpha_{s}\left(Q^{2}\right) \tilde{\gamma}_{f g}^{1}\left(\alpha_{s}\left(Q^{2}\right) / N\right)+\alpha_{s}\left(Q^{2}\right) C_{2,1}^{g}\left(\alpha_{s}\left(Q^{2}\right) / N\right) \tilde{\gamma}_{g g}^{0}\left(\alpha_{s}\left(Q^{2}\right) / N\right)\right) \times \\
\frac{3}{2 N_{f}}\left(\frac{C_{L, 1,0}^{g}}{C_{L, 1}^{g}\left(\alpha_{s}\left(Q^{2}\right) / N\right)}\right)\left(\hat{F}_{L, 0}(N)+\tilde{\hat{F}}_{L, 0}\left(N, Q_{I}^{2}\right)-\left(\frac{36-8 N_{f}}{27}\right) F_{2,0}(N)\right) \times \\
\exp \left[\Phi_{0}^{+}\left(Q^{2}, Q_{I}^{2}\right)+\Phi_{1}^{+}\left(Q_{I}^{2}, Q^{2}\right)\right]-\alpha_{s}\left(Q^{2}\right) \frac{4}{9} \frac{2 N_{f}}{6 \pi b_{0}} F_{2,0}(N) \exp \left[\Phi_{1}^{-}\left(Q^{2}, Q_{I}^{2}\right)\right] \\
+ \text { higher order in } \alpha_{s} \text { and/or } N .
\end{gathered}
$$

The factorization scheme independence of this complete expression guarantees that the term $\left(\tilde{\gamma}_{f g}^{1}\left(\alpha_{s}\left(Q^{2}\right) / N\right)+C_{2,1}^{g}\left(\alpha_{s}\left(Q^{2}\right) / N\right) \tilde{\gamma}_{g g}^{0}\left(\alpha_{s}\left(Q^{2}\right) / N\right)\right)\left(C_{L, 1,0}^{g} / C_{L, 1}^{g}\left(\alpha_{s}\left(Q^{2}\right) / N\right)\right)$ is a factorizationscheme-invariant quantity. Indeed, it was shown by Catani and Hautmann that $\left(\tilde{\gamma}_{f g}^{1}\left(\alpha_{s}\left(Q^{2}\right) / N\right)+\right.$ $\left.C_{2,1}^{g}\left(\alpha_{s}\left(Q^{2}\right) / N\right) \tilde{\gamma}_{g g}^{0}\left(\alpha_{s}\left(Q^{2}\right) / N\right)\right)$ and $C_{L, 1}^{g}\left(\alpha_{s}\left(Q^{2}\right) / N\right)$ could each always be expressed in terms of the product of a factorization-scheme- and renormalization-scheme-independent factor (which they calculated) and a scheme-dependent factor, where the scheme-dependent part is the same for both [18]. Using their results it is a trivial matter to find that

$$
\begin{aligned}
& \left.\left(\gamma_{f g}^{1}\left(\alpha_{s}\left(Q^{2}\right) / N\right)+C_{2,1}^{g}\left(\alpha_{s}\left(Q^{2}\right) / N\right) \gamma_{g g}^{0}\left(\alpha_{s}\left(Q^{2}\right) / N\right)\right)\right) \frac{3}{2 N_{f}}\left(\frac{C_{L, 1,0}^{g}}{C_{L, 1}^{g}\left(\alpha_{s}\left(Q^{2}\right) / N\right)}\right) \\
& =\frac{1}{2 \pi}\left(\frac{3}{2} \gamma_{g g}^{0}\left(\alpha_{s}\left(Q^{2}\right) / N\right)+\sum_{n=0}^{\infty}\left(\gamma_{g g}^{0}\left(\alpha_{s}\left(Q^{2}\right) / N\right)\right)^{n}\right)=\alpha_{s}\left(Q^{2}\right) \gamma_{2 L}^{1}\left(\alpha_{s}\left(Q^{2}\right) / N\right),
\end{aligned}
$$

which is clearly both factorization scheme and renormalization scheme independent. Thus, our expression for the explicit part of (4.44) is entirely in terms of factorization-scheme-invariant, and hence physically meaningful quantities, i.e.

$$
\begin{aligned}
-\alpha_{s}^{2}\left(Q^{2}\right) \frac{d F_{2}\left(N, Q^{2}\right)}{d \alpha_{s}\left(Q^{2}\right)}= & \alpha_{s}\left(Q^{2}\right) \tilde{\gamma}_{2 L}^{1}\left(\alpha_{s}\left(Q^{2}\right) / N\right)\left(\hat{F}_{L, 0}(N)+\tilde{\hat{F}}_{L, 0}\left(N, Q_{I}^{2}\right)-\left(\frac{36-8 N_{f}}{27}\right) F_{2,0}(N)\right) \times \\
& \exp \left[\Phi_{0}^{+}\left(Q^{2}, Q_{I}^{2}\right)+\Phi_{1}^{+}\left(Q_{I}^{2}, Q^{2}\right)\right]-\alpha_{s}\left(Q^{2}\right) \frac{4}{9} \frac{2 N_{f}}{6 \pi b_{0}} F_{2,0}(N) \exp \left[\Phi_{1}^{-}\left(Q^{2}, Q_{I}^{2}\right)\right] \\
& + \text { higher order in } \alpha_{s} \text { and/or } N .
\end{aligned}
$$


This expression is analogous to that for $\hat{F}_{L}\left(N, Q^{2}\right)$ in (4.53), except that it has still not been explicitly separated into inputs and evolution terms. In order to do this we must rewrite (4.57) as

$$
\begin{gathered}
-\alpha_{s}^{2}\left(Q^{2}\right) \frac{d F_{2}\left(N, Q^{2}\right)}{d \alpha_{s}\left(Q^{2}\right)}=\alpha_{s}\left(Q_{I}^{2}\right) \tilde{\gamma}_{2 L}^{1}\left(\alpha_{s}\left(Q_{I}^{2}\right) / N\right)\left(\hat{F}_{L, 0}(N)+\tilde{\hat{F}}_{L, 0}\left(N, Q_{I}^{2}\right)-\left(\frac{36-8 N_{f}}{27}\right) F_{2,0}(N)\right) \times \\
\exp \left[\Phi_{0}^{+}\left(Q^{2}, Q_{I}^{2}\right)+\Phi_{1}^{+}\left(Q_{I}^{2}, Q^{2}\right)+\tilde{\Phi}_{1}^{+}\left(Q_{I}^{2}, Q^{2}\right)\right] \\
-\frac{4}{9} \frac{2 N_{f}}{6 \pi b_{0}} \alpha_{s}\left(Q_{I}^{2}\right) F_{2,0}(N) \exp \left[\Phi_{1}^{-}\left(Q^{2}, Q_{I}^{2}\right)-\ln \left(\frac{\alpha_{s}\left(Q_{I}^{2}\right)}{\alpha_{s}\left(Q^{2}\right)}\right)\right]+\text { higher order in } \alpha_{s} \text { and/or } N,
\end{gathered}
$$

where $\tilde{\Phi}_{1}^{+}\left(Q^{2}, Q_{I}^{2}\right)$ is a series of the same form as $\Phi_{1}^{+}\left(Q^{2}, Q_{I}^{2}\right)$, defined as

$$
\tilde{\Phi}_{1}^{+}\left(Q^{2}, Q_{I}^{2}\right)=-\int_{\alpha_{s}\left(Q^{2}\right)}^{\alpha_{s}\left(Q_{I}^{2}\right)} \frac{d}{d \alpha_{s}\left(q^{2}\right)} \ln \left(\alpha_{s}\left(q^{2}\right) \tilde{\gamma}_{2, L}^{1}\left(N, \alpha_{s}\left(q^{2}\right)\right)\right) d \alpha_{s}\left(q^{2}\right) .
$$

It is now clear how we obtain the "leading part" of this expression, i.e. factor out the subleading parts of the evolution, $\left[\Phi_{1}^{+}\left(Q^{2}, Q_{I}^{2}\right)+\tilde{\Phi}_{1}^{+}\left(Q^{2}, Q_{I}^{2}\right)\right]$ (we note that $\tilde{\Phi}_{1}^{+}\left(Q^{2}, Q_{I}^{2}\right)$ is a renormalization-scheme-independent contribution to this subleading evolution), and $\left[\Phi_{1}^{-}\left(Q^{2}, Q_{I}^{2}\right)-\right.$ $\left.\ln \left(\alpha_{s}\left(Q_{I}^{2}\right) / \alpha_{s}\left(Q^{2}\right)\right)\right]$. This leaves us with the leading-order expression

$$
\begin{aligned}
& -\left(\alpha_{s}^{2}\left(Q^{2}\right) \frac{d F_{2}\left(N, Q^{2}\right)}{d \alpha_{s}\left(Q^{2}\right)}\right)_{0}=\alpha_{s}\left(Q_{I}^{2}\right) \tilde{\gamma}_{2 L}^{1}\left(\alpha_{s}\left(Q_{I}^{2}\right) / N\right) \times \\
& \quad\left(\hat{F}_{L, 0}(N)+\tilde{\hat{F}}_{L, 0}\left(N, Q_{I}^{2}\right)-\left(\frac{36-8 N_{f}}{27}\right) F_{2,0}(N)\right) \exp \left(\Phi_{0}^{+}\left(Q^{2}, Q_{I}^{2}\right)\right)-\alpha_{s}\left(Q_{I}^{2}\right) \frac{4}{9} \frac{2 N_{f}}{6 \pi b_{0}} F_{2,0}(N) .
\end{aligned}
$$

Again, this expression is insensitive to changes in starting scale, up to higher order, and using the one-loop coupling constant, is renormalization scheme independent as well as factorization scheme independent.

Finally we consider the expression for $F_{2}\left(N, Q^{2}\right)$ itself. It is now a relatively simple matter to write this in terms of factorization-scheme-independent quantities and in terms of inputs and evolution terms. Using the definitions we have already made and defining $\hat{\Phi}_{1}^{+}\left(Q^{2}, Q_{I}^{2}\right)$ by

$$
\hat{\Phi}_{1}^{+}\left(Q^{2}, Q_{I}^{2}\right)=-\int_{\alpha_{s}\left(Q^{2}\right)}^{\alpha_{s}\left(Q_{I}^{2}\right)} \frac{d}{d \alpha_{s}\left(q^{2}\right)} \ln \left(\frac{\alpha_{s}\left(q^{2}\right) \gamma_{2, L}^{1}\left(\alpha_{s}\left(q^{2}\right) / N\right)}{\gamma_{g g}^{0}\left(N, \alpha_{s}\left(q^{2}\right)\right)}\right) d \alpha_{s}\left(q^{2}\right)
$$

we may factor terms into inputs and evolutions. Also, by construction it is guaranteed that the change of (4.43) at $Q_{I}^{2}$ under a change in starting scale will cancel the change in the evolution in the first term under a change in starting scale up to higher orders. It is also clear that (4.43) at $Q_{I}^{2}$ is both renormalization scheme and factorization scheme independent, as we require, as long as $\tilde{f}_{1}^{S}\left(N, Q_{I}^{2}\right)$ is scheme independent. The requirement that if $Q_{I}^{2}=A_{L L}$ the input reduces to the nonperturbative input $F_{2,0}(N)$ determines $\tilde{f}_{1}^{S}\left(N, Q_{I}^{2}\right)$ uniquely. It must be the scheme-independent 
quantity $-\alpha_{s}\left(Q_{I}^{2}\right)\left(\tilde{\gamma}_{2 L}^{1}\left(\alpha_{s}\left(Q_{I}^{2}\right) / N\right) / \tilde{\gamma}_{g g}^{0}\left(\alpha_{s}\left(Q_{I}^{2}\right) / N\right)\right)\left(\hat{F}_{L, 0}(N)-\left(\frac{36-8 N_{f}}{27}\right) F_{2,0}(N)\right)$. Hence, the input for $F_{2}\left(N, Q^{2}\right)$ is

$$
F_{2}\left(N, Q_{I}^{2}\right)=F_{2,0}(N)+\alpha_{s}\left(Q_{I}^{2}\right) \frac{\tilde{\gamma}_{2 L}^{1}\left(\alpha_{s}\left(Q_{I}^{2}\right) / N\right)}{\tilde{\gamma}_{g g}^{0}\left(\alpha_{s}\left(Q_{I}^{2}\right) / N\right)} \tilde{\hat{F}}_{L, 0}\left(N, Q_{I}^{2}\right)+\text { higher order in } \alpha_{s} \text { and } / \text { or } N
$$

and the expression for $F_{2}\left(N, Q^{2}\right)$ is

$$
\begin{aligned}
F_{2}\left(N, Q^{2}\right)= & \alpha_{s}\left(Q_{I}^{2}\right) \frac{\tilde{\gamma}_{2 L}^{1}\left(N, \alpha_{s}\left(Q_{I}^{2}\right)\right)}{\tilde{\gamma}_{g g}^{0}\left(N, \alpha_{s}\left(Q_{I}^{2}\right)\right)}\left(\hat{F}_{L, 0}(N)+\tilde{\hat{F}}_{L, 0}\left(N, Q_{I}^{2}\right)-\left(\frac{36-8 N_{f}}{27}\right) F_{2,0}(N)\right) \times \\
& \exp \left[\Phi_{0}^{+}\left(Q^{2}, Q_{I}^{2}\right)+\Phi_{1}^{+}\left(Q_{I}^{2}, Q^{2}\right)+\hat{\Phi}_{1}^{+}\left(Q^{2}, Q_{I}^{2}\right)\right]+F_{2,0}(N) \exp \left[\Phi_{1}^{-}\left(Q^{2}, Q_{I}^{2}\right)\right] \\
& -\alpha_{s}\left(Q_{I}^{2}\right) \frac{\tilde{\gamma}_{2 L}^{1}\left(N, \alpha_{s}\left(Q_{I}^{2}\right)\right)}{\tilde{\gamma}_{g g}^{0}\left(N, \alpha_{s}\left(Q_{I}^{2}\right)\right)}\left(\hat{F}_{L, 0}(N)-\left(\frac{36-8 N_{f}}{27}\right) F_{2,0}(N)\right)
\end{aligned}
$$

+ higher order in $\alpha_{s}$ and/or $N$.

Looking at (4.63) it is clear that there is only one formally LO (in our expansion scheme) input multiplied by a LO evolution, and that is $F_{2,0}(N)$ multiplying unity. But this is obviously completely independent of $\alpha_{s}$, and is rather trivial. At next-to-leading order, or equivalently, at leading $-\alpha_{s}$-dependent order, we include the whole of (4.63) except that we factor $\exp \left[\Phi_{1}^{+}\left(q^{2}, Q_{I}^{2}\right)+\right.$ $\left.\hat{\Phi}_{1}^{+}\left(Q^{2}, Q_{I}^{2}\right)\right]$, out of the first term. Hence, at leading- $\alpha_{s}$-dependent order we have

$$
\begin{aligned}
& F_{2,0}\left(N, Q^{2}\right)=F_{2,0}(N) \exp \left[\Phi_{1}^{-}\left(Q^{2}, Q_{I}^{2}\right)\right] \\
& \quad+\alpha_{s}\left(Q_{I}^{2}\right) \frac{\gamma_{2 L}^{1}\left(\alpha_{s}\left(Q_{I}^{2}\right) / N\right)}{\gamma_{g g}^{0}\left(\alpha_{s}\left(Q_{I}^{2}\right) / N\right)}\left(\hat{F}_{L, 0}(N)+\tilde{\hat{F}}_{L, 0}\left(N, Q_{I}^{2}\right)-\left(\frac{36-8 N_{f}}{27}\right) F_{2,0}(N)\right) \exp \left[\Phi_{0}^{+}\left(Q^{2}, Q_{I}^{2}\right)\right] \\
& \quad-\alpha_{s}\left(Q_{I}^{2}\right) \frac{\gamma_{2 L}^{1}\left(\alpha_{s}\left(Q_{I}^{2}\right) / N\right)}{\gamma_{g g}^{0}\left(\alpha_{s}\left(Q_{I}^{2}\right) / N\right)}\left(\hat{F}_{L, 0}(N)-\left(\frac{36-8 N_{f}}{27}\right) F_{2,0}(N)\right) .
\end{aligned}
$$

In this expression there are clearly no terms which mix if we were to make a change in definition of the coupling $\alpha_{s} \rightarrow \alpha_{s}+\epsilon \alpha_{s}^{2}$, and hence we can consider it as a leading-order expression. If the one-loop coupling is used, it is both factorization scheme and renormalization scheme independent.

We now have the full set of LO expressions in the leading- $\ln (1 / x)$ expansion scheme. We could obtain the correct scheme-independent expressions for the structure functions at higher orders within this expansion scheme, but we choose to finish at leading order. The labour required to obtain higher-order expressions becomes progressively greater and we would obtain expressions requiring unknown anomalous dimensions and coefficient functions. Working to NLO we would need to calculate all the NLO evolutions and all the NLO inputs. This would require all the $\tilde{\gamma}_{g, a}^{1}$ 's, $\tilde{\gamma}_{f, a}^{2}$ 's and $C_{i, 2}^{a}$ 's. There is optimism that these NLO terms will soon be known [36], and once this is so the full NLO scheme-independent expressions should be calculated.

We should make some comments about our LO scheme-independent expressions for $F_{L}\left(N, Q^{2}\right)$, $F_{2}\left(N, Q^{2}\right)$ and $\left(d F_{2}\left(N, Q^{2}\right) / d \ln \left(Q^{2}\right)\right)$. First we note that all depend on factorization-schemeindependent combinations of the $\tilde{\gamma}_{g a}^{0}$ 's, $\tilde{\gamma}_{f a}^{1}$ 's and $C_{i, 1}^{a}$ 's (along with the input parton distributions, 
where $g_{0}\left(N, Q_{I}^{2}\right)$ is also factorization scheme dependent). There is, however, unlike in the loop expansion no terribly simple prescription for how one uses the anomalous dimensions and coefficient functions in order to arrive at the expressions. One must simply examine the form of the solutions and factor out subleading parts.

By comparing (4.62) with (4.54) we now see that there is a very direct relationship between the inputs for our two structure functions at small $x$, i.e. we have a definite prediction, up to additive nonperturbative parts which are flat at small $x$, for one in terms of the other, as well as approximate predictions for the form of each. Also comparing with the form of (4.60), these two inputs for the structure functions are directly related to the slope of $\left(d F_{2} / d \ln Q^{2}\right)$ for small $x$ at $Q_{I}^{2}$. We do not yet know at what $Q_{I}^{2}$ it is most appropriate to choose the inputs, but it is a nontrivial requirement that the inputs for the three expressions are of the correct form and related in the above manner at any $Q_{I}^{2}$.

We should also make some mention of why factorization scheme dependence can be very large in this expansion scheme. In order to do this let us consider (4.42) as an example. A representative example of the way in which factorization-scheme-dependent calculations are done is to consider this expression evaluated with $\tilde{\gamma}_{g g}^{0}\left(\alpha_{s}\left(Q^{2}\right) / N\right), \tilde{\gamma}_{f g}^{1}\left(\alpha_{s}\left(Q^{2}\right) / N\right)$ and $C_{L, 1}^{g}\left(\alpha_{s}\left(Q^{2}\right) / N\right)$ known in some particular factorization scheme, but $\tilde{\gamma}_{g g}^{1}\left(\alpha_{s}\left(Q^{2}\right) / N\right)$, which is unknown, either set equal to zero, or guessed by imposing some ansatz such as momentum conservation. As a first comment we consider the input. The input in terms of parton distributions is multiplied by $C_{L, 1}^{g}\left(\alpha_{s}\left(Q_{I}^{2}\right) / N\right)$, a series which, in general, diverges at $N=\lambda\left(Q_{I}^{2}\right)$. Under a transformation of the type (4.29) this series will be multiplied by $\left(U_{g g}^{0}\left(\alpha_{s}\left(Q_{I}^{2}\right) / N\right)\right)^{-1}$. A number of scheme transformations that are considered have the series $U_{g g}^{0}\left(\alpha_{s}\left(Q_{I}^{2}\right) / N\right)$ also becoming singular at $N=\lambda\left(Q_{I}^{2}\right)$ (e.g. [24][25]). This leads to powerlike growth of the form $x^{-1-\lambda\left(Q_{I}^{2}\right)}$ as $x \rightarrow 0$, but the magnitude of the powerlike behaviour and the manner in which in which it is approached depends on the the precise behaviour of the coefficients in the series. Hence, changes in factorization scheme can lead to very marked differences in the form of the gluon at small $x$, or if the gluon is kept roughly constant (e.g. it is attempted to predict the form of the input structure function by assuming a form for the input gluon), to significant changes in the form of $F_{L}\left(x, Q_{I}^{2}\right)$. Very similar considerations also hold for $F_{2}\left(x, Q^{2}\right)$ because the input depends strongly on $C_{2,1,0}^{g}\left(\alpha_{s}\left(Q_{I}^{2}\right) / N\right)$, which transforms in the same way as $C_{L, 1,0}^{g}\left(\alpha_{s}\left(Q_{I}^{2}\right) / N\right)$.

Examining (4.42) we can also see how the evolution may be strongly factorization scheme dependent. As we have already mentioned, the whole of $\Phi_{1}^{+}\left(Q^{2}, Q_{I}^{2}\right)$, including $\gamma_{g g}^{1}\left(\alpha_{s}\left(Q^{2}\right) / N\right)$, must be used in order to obtain a factorization-scheme-independent expression. The integrand in $\Phi_{1}^{+}\left(Q^{2}, Q_{I}^{2}\right)$ is a series which is a power of $\alpha_{s}\left(Q^{2}\right)$ down on the series $\gamma_{g g}^{0}\left(\alpha_{s}\left(Q^{2}\right) / N\right)$. However, in many popular factorization schemes the coefficients in the incomplete, or incorrect series for this integrand are much larger than those in $\gamma_{g g}^{0}\left(\alpha_{s}\left(Q^{2}\right) / N\right.$ ) (helped by the fact that many of the early coefficients in $\gamma_{g g}^{0}\left(\alpha_{s}\left(Q^{2}\right) / N\right)$ are zero), e.g. they commonly behave roughly like $(12 \ln 2 / \pi)^{n} n^{-3 / 4}$, 
whereas the coefficients in $\gamma_{g g}^{0}\left(\alpha_{s}\left(Q^{2}\right) / N\right)$ behave roughly like $(12 \ln 2 / \pi)^{n} n^{-3 / 2}$. Hence, the incorrect $\Phi_{1}^{+}\left(Q^{2}, Q_{I}^{2}\right)$ can have a dominant effect on the evolution. Under changes of factorization scheme the coefficients in the incorrect series can change by amounts similar to their own magnitude. Therefore, the evolution of the structure functions in terms of a given input can appear to have a very strong factorization scheme dependence. Once again, this is true for the evolution of $F_{2}\left(x, Q^{2}\right)$ as well as for $F_{L}\left(x, Q^{2}\right)$ : the influence of the incorrect $\Phi_{1}^{+}\left(Q^{2}, Q_{I}^{2}\right)$ can be more important than that of $\Phi_{0}^{+}\left(Q^{2}, Q_{I}^{2}\right)$ and $\gamma_{2 L}\left(N, \alpha_{s}\left(Q_{I}^{2}\right)\right)$ combined, where for the latter the coefficients in the series are again relatively small.

Alternative calculational procedures can lead to expressions which are not only similar to (4.42)-(4.44) with incorrect or missing $\gamma_{g g}^{1}\left(\alpha_{s}\left(Q^{2}\right) / N\right)$, but which have additional factorizationscheme-dependent terms. These will be formally of higher order than the terms in (4.42)-(4.44), but again can have very large coefficients in the series expansions. This can lead to even more dramatic effects than those outlined above, e.g. [22].

Once $\gamma_{g g}^{1}\left(N, \alpha_{s}\left(Q^{2}\right)\right)$ is known in a given scheme and (4.42)-(4.44) can be calculated correctly there is no guarantee that the correct $\Phi_{1}^{+}\left(Q^{2}, Q_{I}^{2}\right)$ is not larger than $\Phi_{0}^{+}\left(Q^{2}, Q_{I}^{2}\right)$. If this is the case, $\Phi_{1}^{+}\left(Q^{2}, Q_{I}^{2}\right)$ will then have a large, but at least definite, effect. However, because it is a formally NLO correction to the structure functions, if $\Phi_{1}^{+}\left(Q^{2}, Q_{I}^{2}\right)$ is introduced then the full set of NLO expressions, both evolution and input factors, must be calculated at the same time: the correct calculational method respects renormalization scheme independence as well as factorization scheme dependence. This requires many more terms than just $\Phi_{1}^{+}\left(Q^{2}, Q_{I}^{2}\right)$. Hopefully, the complete NLO expression, as well as being factorization scheme independent, will also cause only fairly small changes to the LO expressions.

Having obtained our full set of leading-order expressions, we can also examine the difference between $F_{2}\left(N, Q^{2}\right)$ and $\left(d F_{2}\left(N, Q^{2}\right) / d \ln Q^{2}\right)$ in this expansion scheme. There is a distinction between $F_{2}\left(N, Q^{2}\right)$ and $\left(d F_{2} / d \ln Q^{2}\right)$ even in the usual loop expansion. Differentiating a fixedorder expression for $F_{2}\left(N, Q^{2}\right)$ and using the $\beta$-function evaluated to the appropriate order in $\alpha_{s}$ results in the fixed-order expression for $\left(d F_{2} / d \ln Q^{2}\right)$ plus terms of higher order in $\alpha_{s}\left(Q^{2}\right)$, which depend on the $\beta$-function. The size of these extra terms is of the same order as the renormalization scheme uncertainty, and the distinction between the fixed-order expressions for $F_{2}\left(N, Q^{2}\right)$ and $\left(d F_{2} / d \ln Q^{2}\right)$ is of similar magnitude to the distinction between renormalization schemes. The distinction when using the small- $x$ expansion appears more graphically, and is not only dependent on terms in the $\beta$-function. If we differentiate (4.64) with respect to $\alpha_{s}\left(Q^{2}\right)$ we obtain

$$
\begin{aligned}
-\alpha_{s}^{2}\left(Q^{2}\right) \frac{d F_{2,0}\left(N, Q^{2}\right)}{d \alpha_{s}\left(Q^{2}\right)}= & \alpha_{s}\left(Q_{I}^{2}\right) \tilde{\gamma}_{2 L}^{1}\left(\alpha_{s}\left(Q_{I}^{2}\right) / N\right)\left(\frac{\gamma_{g g}^{0}\left(\alpha_{s}\left(Q^{2}\right) / N\right)}{\gamma_{g g}^{0}\left(\alpha_{s}\left(Q_{I}^{2}\right) / N\right)}\right) \times \\
& \left(\hat{F}_{L, 0}(N)+\tilde{\hat{F}}_{L, 0}\left(N, Q_{I}^{2}\right)-\left(\frac{36-8 N_{f}}{27}\right) F_{2,0}(N)\right) \exp \left[\Phi_{0}^{+}\left(Q^{2}, Q_{I}^{2}\right)\right] \\
& -\alpha_{s}\left(Q^{2}\right) \frac{4}{9} \frac{2 N_{f}}{6 \pi b_{0}} F_{2,0}(N) \exp \left[\Phi_{1}^{-}\left(Q^{2}, Q_{I}^{2}\right)\right] .
\end{aligned}
$$


This is clearly not exactly the same as (4.60), the difference being due to the additional terms $\left(\gamma_{g g}^{0}\left(\alpha_{s}\left(Q^{2}\right) / N\right) / \gamma_{g g}^{0}\left(\alpha_{s}\left(Q_{I}^{2}\right) / N\right)\right)$ in the first term, and the factors $\left(\alpha_{s}\left(Q^{2}\right) / \alpha_{s}\left(Q_{I}^{2}\right)\right)$ and $\exp \left[\Phi_{1}^{-}\left(Q^{2}, Q_{I}^{2}\right)\right]$ in the second term. All of these factors are unity at the boundary of the evolution, and the two expressions are therefore identical in this limit, i.e the inputs are the same. Therefore, it is the evolution terms which are different when comparing (4.60) and (4.65). Writing

$$
\begin{gathered}
\left(\frac{\gamma_{g g}^{0}\left(\alpha_{s}\left(Q^{2}\right) / N\right)}{\gamma_{g g}^{0}\left(\alpha_{s}\left(Q_{I}^{2}\right) / N\right)}\right)=\exp \left[-\int_{\alpha_{s}\left(Q^{2}\right)}^{\alpha_{s}\left(Q_{I}^{2}\right)} \frac{d}{d \alpha_{s}\left(q^{2}\right)} \ln \left(\gamma_{g g}^{0}\left(\alpha_{s}\left(q^{2}\right) / N\right)\right) d \alpha_{s}\left(q^{2}\right)\right] \\
\alpha_{s}\left(Q^{2}\right)=\alpha_{s}\left(Q_{I}^{2}\right) \exp \left[-\ln \left(\frac{\alpha_{s}\left(Q_{I}^{2}\right)}{\alpha_{s}\left(Q^{2}\right)}\right)\right]
\end{gathered}
$$

we see that the terms present in (4.65) but absent in (4.60) are NLO evolution terms. Thus, as in the loop expansion, the difference between the fixed-order expression for $\left(d F_{2} / d \ln Q^{2}\right)$ and the $\ln Q^{2}$ derivative of the fixed-order $F_{2}$ consists of terms of higher order. However, in the leading- $\ln (1 / x)$ expansion this difference exists between even LO expressions, and even at leading order we have to decide which of the two expressions to use (although there is very little difference between the choices in practice). It may be argued that in certain senses $\left(d F_{2}\left(N, Q^{2}\right) / d \ln Q^{2}\right)$ is more natural because it is a "real" perturbative quantity, beginning at first order in $\alpha_{s}$, as does $F_{L}\left(N, Q^{2}\right)$. We will discuss and confirm this choice in $\S 4.4$.

We should also make some comment about longitudinal momentum conservation. The first moment of the parton distributions is interpreted as the fraction of momentum carried by that type of parton, and it is usually required that $f^{S}\left(1, Q^{2}\right)+g\left(1, Q^{2}\right)=1$. For this to be true for all $Q^{2}$ then, of course, $\left(d\left(f^{S}\left(1, Q^{2}\right)+g\left(1, Q^{2}\right)\right) / d \ln Q^{2}\right)=0$, and from the renormalization group equations this is true if

$$
\gamma_{f f}\left(1, \alpha_{s}\left(Q^{2}\right)\right)+\gamma_{g f}\left(1, \alpha_{s}\left(Q^{2}\right)\right)=0, \quad \gamma_{f g}\left(1, \alpha_{s}\left(Q^{2}\right)\right)+\gamma_{g g}\left(1, \alpha_{s}\left(Q^{2}\right)\right)=0
$$

When expanding the anomalous dimensions order by order in $\alpha_{s}\left(Q^{2}\right)$, it is easy to specify that (4.68) be true for the anomalous dimensions at each order, and to define a wide variety of factorization schemes which maintain this. This guarantees that the fraction of momentum carried by the $n_{\mathrm{th}}{ }^{-}$ order parton distributions is conserved at each $n$. Sometimes, all the momentum is designated to be carried by the zeroth order part of the solution, but this need not be the case. Most often the input is implicitly assumed to be the all-orders input. In this case it is true that it must carry all the momentum, but this method destroys the strict ordering of the solution.

The situation is not as simple for the leading $-\ln (1 / x)$ expansion. As can be seen from the matrix in (4.34), the leading-order $\gamma$ contains entirely positive entries for $N=1$, and is clearly not consistent with momentum conservation: $f_{0}^{S}\left(Q^{2}\right)$ carries a constant amount of momentum while that carried by $g_{0}\left(Q^{2}\right)$ is constantly increasing with $Q^{2}$. In a general factorization scheme there is no reason that working to a finite higher order will restore the relationship (4.68). Two general methods 
have been proposed to restore momentum conservation [21], [20], [37].Both methods guarantee momentum conservation only if one truncates the series for the $\gamma^{\prime}$ s at the $\gamma^{1}$ 's and solves the whole renormalization group equation using this truncated $\gamma$. Hence, both prescriptions destroy any sense of ordering the solution correctly. We advocate that the most sensible approach is to obtain a well-ordered solution for the structure functions, i.e. regard a correct treatment of the physical quantities as of paramount importance. Thus, we simply take the hint offered us by the zeroth-order anomalous dimension, and accept the fact that momentum is not conserved order by order in this method of expansion. How badly it appears to be violated, however, will depend very much on which factorization scheme we claim to work in, and we can choose the violation to be negligible. We will discuss this in more detail in $\S 4.5$.

Finally, we can also discuss the relationship between our scheme-independent solutions and the ones which would be obtained using Catani's physical anomalous dimensions. One can solve for the LO structure functions using these effective anomalous dimensions in exactly the same way as we solved for the parton distributions in the previous subsection. In the same way that we have the relationships between the LO anomalous dimensions, (1.1) and (4.27), we have relationships between the effective anomalous dimensions as seen in (3.7). Using these relationships it is straightforward to obtain the analogous expressions to (4.36), (4.37) and (4.38):

$$
\begin{aligned}
& F_{2}\left(N, Q^{2}\right)= \alpha_{s}\left(Q^{2}\right) \frac{\Gamma_{2 L}^{1}\left(\alpha_{s}\left(Q^{2}\right) / N\right)}{\Gamma_{L L}^{0}\left(\alpha_{s}\left(Q^{2}\right) / N\right)}\left(\hat{F}_{L, 0}(N)+\tilde{\hat{F}}_{L, 0}\left(N, Q_{I}^{2}\right)-\left(\frac{36-8 N_{f}}{27}\right) F_{2,0}(N)\right) \times \\
& \exp \left[\int_{\alpha_{s}\left(Q^{2}\right)}^{\alpha_{s}\left(Q_{I}^{2}\right)} \frac{\tilde{\Gamma}_{L L}^{0}\left(\alpha_{s}\left(q^{2}\right) / N\right)}{\alpha_{s}^{2}\left(q^{2}\right)}+\frac{\tilde{\Gamma}_{L L}^{1}\left(\alpha_{s}\left(q^{2}\right) / N\right)-\left(\frac{36-8 N_{f}}{27}\right) \tilde{\Gamma}_{2 L}^{1}\left(\alpha_{s}\left(q^{2}\right) / N\right)}{\alpha_{s}\left(q^{2}\right)} d \alpha_{s}\left(q^{2}\right)\right] \\
&-\tilde{F}_{2,1}\left(N, Q_{I}^{2}\right)+F_{2,0}(N)\left(\frac{\alpha_{s}\left(Q_{I}^{2}\right)}{\alpha_{s}\left(Q^{2}\right)}\right)^{-\frac{4}{9} \frac{2 N_{f}}{6 \pi b_{0}}}+\text { higher order in } \alpha_{s} \text { and/or } N \\
& \hat{F}_{L}\left(N, Q^{2}\right)=\left(\hat{F}_{L, 0}(N)+\tilde{\hat{F}}_{L, 0}\left(N, Q_{I}^{2}\right)-\left(\frac{36-8 N_{f}}{27}\right) F_{2,0}(N)\right) \times \\
& \exp \left[\int_{\alpha_{s}\left(Q^{2}\right)}^{\alpha_{s}\left(Q_{I}^{2}\right)} \frac{\tilde{\Gamma}_{L L}^{0}\left(\alpha_{s}\left(q^{2}\right) / N\right)}{\alpha_{s}^{2}\left(q^{2}\right)}+\frac{\tilde{\Gamma}_{L L}^{1}\left(\alpha_{s}\left(q^{2}\right) / N\right)-\left(\frac{36-8 N_{f}}{27}\right) \tilde{\Gamma}_{2 L}^{1}\left(\alpha_{s}\left(q^{2}\right) / N\right)}{\alpha_{s}\left(q^{2}\right)} d \alpha_{s}\left(q^{2}\right)\right] \\
&+\left(\frac{36-8 N_{f}}{27}\right) F_{2,0}(N)\left(\frac{\alpha_{s}\left(Q_{I}^{2}\right)}{\alpha_{s}\left(Q^{2}\right)}\right)^{-\frac{4}{9} \frac{2 N_{f}}{6 \pi b_{0}}}+\text { higher order in } \alpha_{s} \text { and } / \text { or } N
\end{aligned}
$$


and

$$
\begin{array}{r}
-\alpha_{s}^{2}\left(Q^{2}\right) \frac{d F_{2}\left(N, Q^{2}\right)}{d \alpha_{s}\left(Q^{2}\right)}= \\
\exp \left[\int_{\alpha_{s}\left(Q^{2}\right)}^{\alpha_{s}\left(Q_{I}^{2}\right)} \frac{\left.\tilde{\Gamma}_{L L}^{0}\left(\alpha_{s}\right) \tilde{\Gamma}_{2 L}^{1}\left(q_{s}^{2}\right) / N\right)}{\alpha_{s}^{2}\left(q^{2}\right)}+\frac{\tilde{\Gamma}_{L L}^{1}\left(\alpha_{s}^{2}\left(q^{2}\right) / N\right)-\left(\frac{36-8 N_{f}}{27}\right) \tilde{\Gamma}_{2 L}^{1}\left(\alpha_{s}\left(q^{2}\right) / N\right)}{\alpha_{s}\left(q^{2}\right)} d \alpha_{s}\left(q^{2}\right)\right] \\
-\frac{4}{9} \alpha_{s}\left(Q^{2}\right) F_{2,0}(N) \frac{2 N_{f}}{6 \pi b_{0}}\left(\frac{\alpha_{s}\left(Q_{I}^{2}\right)}{\alpha_{s}\left(Q^{2}\right)}\right)^{-\frac{4}{9} \frac{2 N_{f}}{6 \pi b_{0}}}+\text { higher order in } \alpha_{s} \text { and /or } N,
\end{array}
$$

where $\tilde{\hat{F}}_{L, 0}\left(N, Q_{I}^{2}\right)$ is a function of $\Gamma_{L L}^{0}$ rather than $\gamma_{g g}^{0}$.

We see that, once we make the identifications

$$
\gamma_{g g}^{0}\left(\alpha_{s}\left(Q^{2}\right) / N\right)=\Gamma_{L L}^{0}\left(\alpha_{s}\left(Q^{2}\right) / N\right), \quad \gamma_{2 L}^{1}\left(\alpha_{s}\left(Q^{2}\right) / N\right)=\Gamma_{2 L}^{1}\left(\alpha_{s}\left(Q^{2}\right) / N\right)
$$

and

$$
\begin{aligned}
\tilde{\gamma}_{g g}^{1}\left(\alpha_{s}\left(Q^{2}\right) / N\right)+\frac{4}{9} \tilde{\gamma}_{f g}^{1}\left(\alpha_{s}\left(Q^{2}\right) / N\right)- & \frac{d}{d \alpha_{s}\left(Q^{2}\right)}\left(\ln \left(\frac{C_{L, 1}^{g}\left(\alpha_{s}\left(Q^{2}\right) / N\right)}{C_{L, 1,0}^{g}}\right)\right) \\
& =\tilde{\Gamma}_{L L}^{1}\left(\alpha_{s}\left(Q^{2}\right) / N\right)-\left(\frac{36-8 N_{f}}{27}\right) \tilde{\Gamma}_{2 L}^{1}\left(\alpha_{s}\left(Q^{2}\right) / N\right),
\end{aligned}
$$

(4.70) is identical to (4.53), (4.71) is identical to (4.57) and (4.69) is identical to (4.63). Of course, the identifications (4.72)-(4.73) are exactly what we obtain from the definitions of the physical anomalous dimensions in $\S 3$.

Thus, we are able to reach these expressions for the structure functions somewhat more directly by using the physical anomalous dimensions, and do not have to worry about problems with factorization scheme dependence (though we do have to calculate the physical anomalous dimensions in terms of known coefficient functions and anomalous dimensions of course). Once we have obtained these expressions using the physical anomalous dimensions we may then separate each of the terms into input parts and evolution parts (where more of this, but certainly not all, has already been done automatically when using the physical anomalous dimensions) and keep the most leading parts, obtaining once again the LO expressions (4.54), (4.60) and (4.64). So, using the physical anomalous dimensions leads us in a rather more direct manner to the correct leadingorder expressions. If we were to work to higher orders, the amount of simplification obtained by using the physical anomalous dimensions rather than working in terms of parton densities would increase significantly.

However, we stress that one will always automatically obtain factorization-schemeindependent answers by working to well-defined orders in physical quantities even when working in terms of partons. Also, we stress very strongly that even if one uses the physical anomalous dimensions, care is still needed to obtain expressions which are consistent with renormalization scheme 
dependence, and there is no simple prescription for obtaining the correct results even in terms of the physical anomalous dimensions. If we were to solve the evolution equations for structure functions using $\tilde{\Gamma}_{L L}^{0}, \tilde{\Gamma}_{L 2}^{0}, \tilde{\Gamma}_{2 L}^{1}$ and $\tilde{\Gamma}_{22}^{1}$ we would obtain the explicit parts of (4.69)-(4.71) (with $\tilde{\Gamma}_{L L}^{1}=0$ ) plus corrections of higher order. Terms must still be factored out of these expressions in order to obtain the true leading order structure functions. We will discuss the phenomenological consequences of this in $\S 6$.

In this section, we have derived well-ordered, and consequently, factorization-schemeindependent expressions for structure functions in the leading- $\ln (1 / x)$ expansion (which should be useful at small $x$ ) up to the order which is useful at present. This expansion does, however, sacrifice any attempt to describe the structure functions at large $x$. We would hope there is some expansion scheme which will be useful at all values of $x$. In the next section we will show that there is indeed an expansion scheme which satisfies this criterion, and argue that it is the only really correct expansion scheme.

\subsection{The Renormalization-Scheme-Consistent Expansion.}

In order to devise an expansion scheme which is useful at both large and small $x$ we would $a$ priori expect that we would need to use anomalous dimensions and coefficient functions at low orders in both $\alpha_{s}$ and the leading $-\ln (1 / x)$ expansion. There have already been various methods along these lines, however, they have all been scheme dependent. We demand complete consistency of our expressions for physical quantities with renormalization scheme invariance (which then guarantees factorization scheme independence). Consequently, our approach will be different from those used previously, and the results and conclusions will also be somewhat different.

To begin, let us consider what we have meant by "consistency with renormalization scheme dependence" so far in this paper. In both the loop expansion and the leading- $\ln (1 / x)$ expansion we demanded that once we had chosen a particular renormalization scheme and chosen to work to a particular order in this renormalization scheme then we would include all terms in our expressions for the structure function which were of greater magnitude than the uncertainty due to the freedom of choice of renormalization scheme (i.e. the uncertainty in the definition of the coupling constant), and no others. In each case the leading-order term consisted of the lowest-order inputs multiplying the lowest-order evolution terms. If working with the $n$-loop coupling constant the uncertainty in its definition is of order $\alpha_{s}^{n+1}$. Thus, the uncertainty of the input or evolution when working to $n_{\text {th }}$-order is the change in the leading-order input or evolution if $\alpha_{s} \rightarrow \alpha_{s}\left(1+\epsilon \alpha_{s}^{n}\right)$. Hence, the uncertainty in the whole structure function is of the order of the change of the leading-order part under such a change in the coupling. Therefore the $n_{\mathrm{th}}$-order renormalization-scheme-independent expression includes all complete terms smaller than this change. 
This definition does give us a well-defined way of building up an ordered solution to the structure functions, but relies upon the definition of a given expansion scheme. It leaves an ambiguity about how we define the leading-order expressions and in how we define the order of terms compared to this leading-order term. Our two examples, the loop expansion, where the size of a term is determined simply by its order in $\alpha_{s}$, and the leading $-\ln (1 / x)$ expansion, where $\ln (1 / x)$ is put on an equal footing to $\alpha_{s}$, are just two examples of expansion schemes. Both have potential problems: in the former one does not worry about the large- $\ln (1 / x)$ terms which can cause enhancement at small $x$ of terms which are higher order in $\alpha_{s}$, and in the latter one does not worry about the fact that at large $x$, especially as $Q^{2}$ increases, it is the terms of lowest order in $\alpha_{s}$ that dominant. Hence, both should have limited regions of validity.

The shortcomings of these two expansion schemes come about because, even though any given order contains no terms which are inconsistent with working to the same given order in a particular renormalization scheme, in neither case does it include every one of the terms which are consistent to working to a given order in the renormalization scheme. In each expansion scheme some of the terms appearing at what we call higher orders are not really subleading in $\alpha_{s}$ to any terms which have already appeared. Thus, despite the fact that for a given expansion method these terms are defined to be the same order as uncertainties due to the choice of renormalization scheme, they are not terms which can actually be generated by a change in renormalization scheme. ${ }^{11}$

In order to demonstrate this point more clearly we consider a simple toy model. Let us imagine some hypothetical physical quantity which can be expressed in the form

$$
H\left(N, \alpha_{s}\left(Q^{2}\right)\right)=\sum_{m=1}^{\infty} \alpha_{s}\left(Q^{2}\right) \sum_{n=-m}^{\infty} a_{m n} N^{n} \equiv \sum_{i=0}^{\infty} \alpha_{s}^{i}\left(Q^{2}\right) \sum_{j=1-i}^{\infty} b_{i j}\left(\frac{\alpha_{s}\left(Q^{2}\right)}{N}\right)^{j}
$$

where the expansion in powers of $N$ about $N=0$ is convergent for all $N$. The first way of writing $H\left(N, \alpha_{s}\left(Q^{2}\right)\right)$ as a power series corresponds to the loop expansion, where we work order by order in $m$, out to $m=k$, and use the $k$-loop coupling. The second corresponds to the leading- $\ln (1 / x)$ expansion where we work order by order in $i$, out to $i=l$, and use the $(l+1)$-loop coupling. Let us, for a moment, consider the LO expression in the loop expansion, $\alpha_{s}\left(Q^{2}\right) \sum_{n=-1}^{\infty} a_{1 n} N^{n}$. The coupling is uncertain by $\mathcal{O}\left(\alpha_{s}^{2}\left(Q^{2}\right)\right)$ and hence the uncertainty of the leading-order expression (i.e. the change due to a change of the coupling) is $\sim \alpha_{s}^{2}\left(Q^{2}\right) \sum_{n=-1}^{\infty} b_{1 n} N^{n}$. There is no change with powers of $N$ less than -1 , and hence any such term is not really subleading. Similarly, the uncertainty of the leading-order expression in the leading $\ln (1 / x)$ expansion contains no terms at first order in $\alpha_{s}$ (or with positive powers of $N$ ), and such terms are not really subleading either. The full set of terms contained within the combination of both leading-order expressions is genuinely leading order, and is therefore renormalization scheme independent by definition.

11 Similarly, they cannot be generated by a change in renormalization scale. 
Perhaps the best way in which to write our expression for $H\left(N, \alpha_{s}\left(Q^{2}\right)\right)$ in order to appreciate these points is

$$
H\left(N, \alpha_{s}\left(Q^{2}\right)\right)=\sum_{m=-1}^{\infty} N^{m} \sum_{n=1}^{\infty} c_{m n} \alpha_{s}^{n}\left(Q^{2}\right)+\sum_{m=2}^{\infty} N^{-m} \sum_{n=m}^{\infty} c_{m n} \alpha_{s}^{n}\left(Q^{2}\right)
$$

i.e. as an infinite number of power series in $\alpha_{s}\left(Q^{2}\right)$, one for each power on $N$. Each of these series in $\alpha_{s}\left(Q^{2}\right)$ is independent of the others, and the lowest order in $\alpha_{s}\left(Q^{2}\right)$ of each is therefore renormalization scheme independent and part of the complete LO expression for $H\left(N, \alpha_{s}\left(Q^{2}\right)\right)$. The full LO expression for $H\left(N, \alpha_{s}\left(Q^{2}\right)\right)$ is therefore

$$
\begin{aligned}
H_{0}\left(N, \alpha_{s}\left(Q^{2}\right)\right) & =\sum_{m=-1}^{\infty} N^{m} c_{m 1} \alpha_{s}\left(Q^{2}\right)+\sum_{m=2}^{\infty} c_{m m} N^{-m} \alpha_{s}^{m}\left(Q^{2}\right) \\
& \equiv \alpha_{s}\left(Q^{2}\right) \sum_{n=-1}^{\infty} a_{0 n} N^{n}+\sum_{j=2}^{\infty} b_{0 j}\left(\frac{\alpha_{s}\left(Q^{2}\right)}{N}\right)^{j} .
\end{aligned}
$$

Hence, the combined set of terms considered LO in the two expansion schemes comprise the full set of renormalization scheme invariant, and thus truly leading-order, terms. By considering $H\left(N, \alpha_{s}\left(Q^{2}\right)\right)$ written in the form (4.75), and considering how the coefficients in the expression must change in order to make the whole expression invariant under a redefinition of the coupling constant, $\alpha_{s}\left(Q^{2}\right) \rightarrow \alpha_{s}\left(Q^{2}\right)+\mathcal{O}\left(\alpha_{s}^{m}\left(Q^{2}\right)\right)$, we see that the $n_{\mathrm{th}}$-order expression for $H\left(N, \alpha_{s}\left(Q^{2}\right)\right)$, which should be used with the $n$-loop coupling constant, consists of the sum of the first $n$ terms in each of the power series in $\alpha_{s}\left(Q^{2}\right)$. Thus, the full $n_{\mathrm{th}}$-order expression consists of the $n_{\mathrm{th}}$-order expression in the loop expansion plus additional terms with inverse powers of $N$ greater than $n$.

Similar arguments have already been applied to the anomalous dimensions and coefficient functions, for example [18] and particularly [20], though in a somewhat weaker form. Here we take a strong, inflexible viewpoint and insist that the complete renormalization-scheme-consistent expressions (with no artificial suppression of leading- $\ln (1 / x)$ terms [20]), must be used. Furthermore, and very importantly, the expressions used must be those for the physical structure functions, not for the factorization-scheme- and renormalization-scheme-dependent coefficient functions and anomalous dimensions.

When considering the real structure functions the situation is technically a great deal more complicated than our toy model, but the principle is exactly the same. This can be seen by examining the the LO expressions for the structure functions in the two expansion schemes already considered. There is some overlap between the two, but each contains an infinite number of terms not present in the other. Considering the change of each leading-order expression under a change of coupling of $\mathcal{O}\left(\alpha_{s}^{2}\right)$ we see that, as with our toy model, the change in the LO structure functions in the loop expansion contains no terms in the LO expressions in the leading $-\ln (1 / x)$ expansion, and vice versa i.e., none of the terms contained within each of the LO expressions are generated 
by uncertainties at higher order in the other. Therefore, they should really all be regarded as genuinely LO, and be included in the full "leading order" expressions for the structure functions which use the one-loop coupling constant. Since these expressions contain all the parts of the oneloop expressions, and also contain leading $-\ln (1 / x)$ terms as well, they should be able to describe the data over the full range of parameter space (except very low $Q^{2}$, of course), as we would like from our correct LO expressions. However, there are two main complications when considering structure functions in comparison to our simple toy model. One is that the structure functions are combinations of evolution parts and input parts, rather than one simple power series in $\alpha_{s}\left(Q^{2}\right)$. The other is that in general the physical anomalous dimensions, out of which the perturbative parts are constructed, are nonanalytic functions which cannot be expressed as power series about a particular value $N_{0}$ for all $N$. They have singularities at $N=0$, and also at negative powers of $N$ (as well as possible $\alpha_{s}\left(Q^{2}\right)$-dependent nonanalyticities due to resummation effects, e.g. the branch point in $\Gamma_{L L}^{0}\left(N, \alpha_{s}\left(Q^{2}\right)\right)$ at $\left.N=\lambda\left(Q^{2}\right)\right)$. We will deal with this second complication first.

Let us consider the perturbative parts of the expressions for the structure functions. The singularities at negative integer values of $N$ mean that we cannot write any physically meaningful quantity as just a power series about $N=0$ (or about $(N+1)$ for the nonsinglet case). Any such power series expansion will have a radius of convergence of unity, and a series expansion which applies over the whole range of $N$ does not exist. In order to overcome this problem let us consider making the inverse transformation of some physically relevant perturbative function $A\left(N, \alpha_{s}\left(Q^{2}\right)\right)$ to $x$-space. The inverse of the Mellin transformation (2.1) is

$$
\mathcal{A}\left(x, \alpha_{s}\left(Q^{2}\right)\right)=\frac{1}{2 \pi i} \int_{c-i \infty}^{c+i \infty} \exp [\xi N] A\left(N, \alpha_{s}\left(Q^{2}\right)\right) d N
$$

where the line of integration is to the right of all nonanalyticities, and $\xi=\ln (1 / x)$. Since $A\left(N, \alpha_{s}\left(Q^{2}\right)\right)$ has, in general, singularities for all nonpositive integers, this whole integral may be evaluated by performing an infinite series of integrals, each with a contour centred on a given singularity, and not extending as far as unity from this singularity, i.e. not reaching any of the other singularities. Within each of these contours the function $A\left(N, \alpha_{s}\left(Q^{2}\right)\right)$ may be expanded as a power series about the singularity, i.e. we may write

$$
\mathcal{A}\left(x, \alpha_{s}\left(Q^{2}\right)\right)=\frac{1}{2 \pi i} \sum_{n=0}^{\infty} x^{n} \int_{c_{n}} \exp [\xi(N+n)] A_{n}\left((N+n), \alpha_{s}\left(Q^{2}\right)\right) d(N+n),
$$

where $A_{n}\left((N+n), \alpha_{s}\left(Q^{2}\right)\right)$ denotes $A\left(N, \alpha_{s}\left(Q^{2}\right)\right)$ expanded as a power series about $N=-n$. The integrals will produce functions of $\xi$, which do not sum to integer powers of $x$, and each of the integrals in (4.78) will be independent and physically relevant in its own right. Hence, we must consider the complete moment-space expression as an infinite number of expressions of the form (4.75), each one having power series expansions in terms of $(N+n)$, where $n=0 \rightarrow \infty$. The 
expression for each $n$ is then related to the part of the $x$-space expression behaving $\sim x^{n}$. Of course, in practice, unless we want to examine the details of the structure function for $x$ very close to 1 , we can ignore all $n$ greater than a finite, relatively small constant.

Thus, when calculating the expressions for the perturbative part of the singlet structure functions, we are only concerned about LO terms beyond lowest order in $\alpha_{s}$ for the specific case of $n=0$. For $n>0$ we take the whole LO expression to be the one-loop expression. The terms we ignore by making this necessary decision are those which are LO in $\ln (1 / x)$ at first order in $x$. Although these terms grow like $\alpha_{s}^{m} \ln ^{2 m-1}(1 / x)$, there is no evidence that their coefficients are any larger than those for the zeroth-order-in- $x$ logarithms. Since the resummed terms at zeroth order in $x$ only begin to make an impact as $x$ falls to $\sim 0.1$ (as we will see), and only become dominant for $x$ much smaller than this, the effect of terms like $x \alpha_{s}^{m} \ln ^{2 m-1}(1 / x)$ should be very small in comparison. Indeed, the effect of those terms of the form $x \alpha_{s}^{m} \ln ^{2 m-1}(1 / x)$ which are actually known, i.e. $m=2$, are indeed negligible. In a similar manner, we only consider the one-loop expressions for the nonsinglet structure functions in practice: the other LO parts of the expressions again lead to small- $x$ enhancement of the form $x \alpha_{s}^{m} \ln ^{2 m-1}(1 / x)$, which is very small compared to the singlet small- $x$ enhancement, and there is only detailed data at very small $x$ for the total structure function.

We now return to our first problem, i.e. the fact that the structure functions are expressed in terms of both inputs and evolution parts. We discuss the case of the nonsinglet structure functions as an example first.

\subsection{The Renormalization-Scheme-Consistent (RSC) Nonsinglet Structure Functions.}

We consider a nonsinglet longitudinal structure function. For the nonsinglet structure functions the physical anomalous dimensions contain no singularities at $N=0$, so the leading- $\ln (1 / x)$ behaviour comes from singularities at $N=-1$. Expanding about $N=-1$, the full LO physical anomalous dimension can be written in the form,

$$
\begin{aligned}
\Gamma_{L, 0}^{N S}\left(N+1, \alpha_{s}\left(Q^{2}\right)\right)=\alpha_{s}\left(Q^{2}\right) & {\left[\sum_{m=-1}^{\infty} a_{m}(N+1)^{m}\right.} \\
& \left.+\sum_{m=1}^{\infty} b_{m}(N+1)^{-1}\left(\frac{\alpha_{s}\left(Q^{2}\right)}{(N+1)^{2}}\right)^{m}+\sum_{m=1}^{\infty} c_{m}\left(\frac{\alpha_{s}\left(Q^{2}\right)}{(N+1)^{2}}\right)^{m}\right] .
\end{aligned}
$$

The first sum is just $\Gamma_{L, 0, l}^{N S}(N+1)$, the one-loop anomalous dimension expanded in powers of $(N+1)$. The second sum contains the leading singularities in $(N+1)$ for all other orders in $\alpha_{s}\left(Q^{2}\right)$. The final sum is included because, despite the obvious fact that it is a power of $(N+1)^{-1}$ down on the second sum, a series of this form cannot be created from the second sum by a change in the definition of the coupling of $\mathcal{O}\left(\alpha_{s}^{2}\left(Q^{2}\right)\right)$. Therefore, the third sum is not subleading in $\alpha_{s}\left(Q^{2}\right)$ to 
the second sum, and must be renormalization scheme independent. Integrating (4.79) between $Q_{I}^{2}$ and $Q^{2}$, and including the overall power of $\alpha_{s}\left(Q^{2}\right)$ for the longitudinal structure function we obtain the leading-order evolution

$$
\left(\frac{\alpha_{s}\left(Q_{I}^{2}\right)}{\alpha_{s}\left(Q^{2}\right)}\right)^{\tilde{\Gamma}_{L, 0, l}^{N S}(N+1)-1} \exp \left[\int_{\alpha_{s}\left(Q^{2}\right)}^{\alpha_{s}\left(Q_{I}^{2}\right)}\left(\sum_{m=1}^{\infty}\left(\frac{1}{N+1} b_{m}+c_{m}\right)\left(\frac{\alpha_{s}\left(q^{2}\right)}{(N+1)^{2}}\right)^{m}\right) \frac{d \alpha_{s}\left(q^{2}\right)}{b_{0} \alpha_{s}^{2}\left(q^{2}\right)}\right] .
$$

In order to construct the full LO input we must consider how the evolution term (4.80) changes under a change in starting scale, and therefore how the input must change in order to compensate for this. With a little work we can see that the full LORSC input is

$$
\begin{aligned}
& \alpha_{s}\left(Q_{I}^{2}\right) F_{2,0}^{N S}(N)\left(C_{L, 1, l}^{N S}(N+1)+C_{L, 1, l}^{N S}(N=-1)\left(\ln \left(Q_{I}^{2} / A_{L}^{N S}\right) \alpha_{s}\left(Q_{I}^{2}\right) \times\right.\right. \\
& \left.\left.\quad\left(\frac{1}{N+1} \sum_{m=0}^{\infty} b_{m}\left(\frac{\alpha_{s}\left(Q_{I}^{2}\right)}{(N+1)^{2}}\right)^{m}+\sum_{m=0}^{\infty}\left(c_{m}+\frac{1}{2} \sum_{n=0}^{m} b_{n} b_{m-n} \ln \left(Q_{I}^{2} / A_{L}^{N S}\right)\right)\left(\frac{\alpha_{s}\left(Q_{I}^{2}\right)}{(N+1)^{2}}\right)^{m}\right)\right)\right),
\end{aligned}
$$

where $b_{0} \equiv a_{-1}$ and $c_{0}=0$. The first term is just the lowest-order input in the loop expansion, while the second includes all the leading- $\ln (1 / x)$ terms.

Hence, the full LORSC expression for the nonsinglet longitudinal structure function, expanded about $N=-1$, is

$$
\begin{gathered}
F_{L, R S C, 0}^{N S}\left(N, Q^{2}\right)=\alpha_{s}\left(Q_{I}^{2}\right) F_{2,0}^{N S}(N)\left(C_{L, 1, l}^{N S}(N+1)+C_{L, 1, l}^{N S}(N=-1)\left(\ln \left(Q_{I}^{2} / A_{L}^{N S}\right) \alpha_{s}\left(Q_{I}^{2}\right) \times\right.\right. \\
\left.\left.\left(\frac{1}{N+1} \sum_{m=0}^{\infty} b_{m}\left(\frac{\alpha_{s}\left(Q_{I}^{2}\right)}{(N+1)^{2}}\right)^{m}+\sum_{m=0}^{\infty}\left(c_{m}+\frac{1}{2} \sum_{n=0}^{m} b_{n} b_{m-n} \ln \left(Q_{I}^{2} / A_{L}^{N S}\right)\right)\left(\frac{\alpha_{s}\left(Q_{I}^{2}\right)}{(N+1)^{2}}\right)^{m}\right)\right)\right) \times \\
\left(\frac{\alpha_{s}\left(Q_{I}^{2}\right)}{\alpha_{s}\left(Q^{2}\right)}\right)^{\tilde{\Gamma}_{L, 0, l}^{N S}(N+1)-1} \exp \left[\int_{\alpha_{s}\left(Q^{2}\right)}^{\alpha_{s}\left(Q_{I}^{2}\right)}\left(\sum_{m=1}^{\infty}\left(\frac{1}{N+1} b_{m}+c_{m}\right)\left(\frac{\alpha_{s}\left(q^{2}\right)}{(N+1)^{2}}\right)^{m}\right) \frac{d \alpha_{s}\left(q^{2}\right)}{b_{0} \alpha_{s}^{2}\left(q^{2}\right)}\right]
\end{gathered}
$$

In practice, we will only use the one-loop expression for the nonsinglet structure functions for all the singularities in the anomalous dimensions. This is because of the phenomenological reasons given at the end of the last subsection, and also because of lack of knowledge of the full physical anomalous dimensions. The terms $\sim \alpha_{s}^{m}(N+1)^{2 m-1}$ in the anomalous dimension are all known [38], however, there is little knowledge yet of the terms of the sort $\sim \alpha_{s}^{m}(N+1)^{2 m-2}$. These are an intrinsic part of the LORSC expression for the nonsinglet structure function, and should be calculated and included in order to give a true indication of the effect of leading $-\ln (1 / x)$ terms. Hence, we believe that calculations of the nonsinglet [23][39] (and polarized [40]) structure functions which claim to include leading- $\ln (1 / x)$ corrections are presently incomplete.

We now consider the nonsinglet structure function $F_{2}^{N S}\left(N, Q^{2}\right)$. This leads us back to our previous question of whether we should use the full RSC expression for $F_{2}\left(N, Q^{2}\right)$ or that for 
$\left(d F_{2}\left(N, Q^{2}\right) / d \ln Q^{2}\right)$. In order to illustrate the difference between the two we consider the simpler nonsinglet case, and also pretend for the moment that there is no small- $x$ enhancement at higher orders in $\alpha_{s}$. Hence, the LO term in the evolution is just $\left(\frac{\alpha_{s}\left(Q_{I}^{2}\right)}{\alpha_{s}\left(Q^{2}\right)}\right)^{\tilde{\Gamma}_{2,0, l}^{N S}(N+1)}$. The input may be written as a power series in $\alpha_{s}\left(Q_{I}^{2}\right)$, as we saw in $\S 4.1$, and is of the form

$$
F_{2,0}^{N S}\left(N, Q_{I}^{2}\right)=F_{2,0}^{N S}(N)\left[1+\alpha_{s}\left(Q_{I}^{2}\right) \ln \left(Q_{I}^{2} / A_{2}^{N S}\right) \Gamma_{N S}^{2,0, l}(N+1)+\text { higher order in } \alpha_{s}\left(Q_{I}^{2}\right)\right]
$$

Therefore, as well as the lowest order $\alpha_{s^{-}}$, and hence $Q_{I}^{2}$-dependent part of the input there is also the "sub-lowest-order", $Q_{I}^{2}$-independent part. These two terms are clearly of different order, but under a change in renormalization scheme, both remain unchanged and both should therefore appear in the LO definition of the structure function. This mixing of orders seems rather unsatisfactory, and comes about because for $F_{2}^{N S}\left(N, Q^{2}\right)$ the structure function still exists at zeroth order in $\alpha_{s}$ and hence, it is not a perturbative quantity in quite the sense way as $F_{L}^{N S}\left(N, Q^{2}\right)$ or $\left(d F_{2}^{N S}\left(N, Q^{2}\right) / d \ln Q^{2}\right)$, both of which vanish with $\alpha_{s}$. So in our simplified model the LORSC expression for $F_{2}^{N S}\left(N, Q^{2}\right)$, obtained by combining the LO input and evolution, is

$$
F_{2, R S C, 0}^{N S}\left(N, Q^{2}\right)=F_{2,0}^{N S}(N)\left[1+\alpha_{s}\left(Q_{I}^{2}\right) \ln \left(Q_{I}^{2} / A_{2}^{N S}\right) \Gamma_{N S}^{2,0, l}(N+1)\right]\left(\frac{\alpha_{s}\left(Q_{I}^{2}\right)}{\alpha_{s}\left(Q^{2}\right)}\right)^{\tilde{\Gamma}_{2,0, l}^{N S}(N+1)} .
$$

This consists of two parts which are clearly of different magnitude, and it is clear that the same effect will be seen for the singlet structure function.

If we instead consider $\left(d F_{2}^{N S}\left(N, Q^{2}\right) / d \ln Q^{2}\right)$ the full expression is

$$
\begin{aligned}
\frac{d F_{2}^{N S}\left(N, Q^{2}\right)}{d \ln Q^{2}}= & \Gamma_{2, N S}\left(N+1, \alpha_{s}\left(Q_{I}^{2}\right)\right) F_{2}^{N S}\left(N, Q_{I}^{2}\right) \times \\
& \exp \left[\int_{\ln Q_{I}^{2}}^{\ln Q^{2}}\left(\Gamma_{2, N S}\left(N+1, \alpha_{s}\left(q^{2}\right)\right)-\frac{d}{d \ln q^{2}} \ln \left(\Gamma_{2, N S}\left(N+1, \alpha_{s}\left(q^{2}\right)\right)\right)\right) d \ln q^{2}\right] .
\end{aligned}
$$

Hence, the input may be written as

$$
\begin{aligned}
\left(\frac{d F_{2,0}^{N S}\left(N, Q^{2}\right)}{d \ln Q^{2}}\right)_{Q_{I}^{2}}=F_{2,0}^{N S}(N)\left[\alpha_{s}\left(Q_{I}^{2}\right) \Gamma^{2,0, l}(N+1)+\alpha_{s}^{2}\left(Q_{I}^{2}\right)\right. & \left(\ln \left(Q_{I}^{2} / A_{2}^{N S}\right)\left(\Gamma_{N S}^{2,0, l}(N+1)\right)^{2}\right. \\
+ & \left.\left.+\Gamma^{2,1, l}(N+1)\right)+\mathcal{O}\left(\alpha_{s}^{3}\left(Q_{I}^{2}\right)\right)\right]
\end{aligned}
$$

The $\mathcal{O}\left(\alpha_{s}^{2}\left(Q_{I}^{2}\right)\right)$ piece is renormalization scheme dependent, so this time we have a LORSC input which is of a given order in $\alpha_{s}\left(Q_{I}^{2}\right)$. The full LO expression for $\left(d F_{2}\left(N, Q^{2}\right) / d \ln Q^{2}\right)$ is then

$$
\left(\frac{d F_{2,0}^{N S}\left(N, Q^{2}\right)}{d \ln Q^{2}}\right)_{0}=F_{2,0}^{N S}(N) \alpha_{s}\left(Q_{I}^{2}\right) \Gamma_{2,0, l}^{N S}(N+1)\left(\frac{\alpha_{s}\left(Q_{I}^{2}\right)}{\alpha_{s}\left(Q^{2}\right)}\right)^{\tilde{\Gamma}_{2,0, l}^{N S}(N+1)-1} .
$$


This is rather more satisfactory than the renormalization-scheme-independent expression for $F_{2}^{N S}\left(N, Q^{2}\right)$ itself (4.84), and hence we choose $\left(d F_{2}\left(N, Q^{2}\right) / d \ln Q^{2}\right)$ to be the perturbative quantity we calculate, in both the nonsinglet and singlet case.

If we wish to calculate the structure function $F_{2}\left(N, Q^{2}\right)$ itself to a given order we will do this by integrating the given order expression for $\left(d F_{2}\left(N, Q^{2}\right) / d \ln Q^{2}\right)$ between $Q_{I}^{2}$ and $Q^{2}$, and adding it to $F_{2}\left(N, Q_{I}^{2}\right)$ evaluated to the same order. For example, in our simplified nonsinglet model we would integrate (4.87) and add this to the explicitly written part of (4.83). This results in the effective LO expression

$$
F_{2, R S C, 0}^{N S}\left(N, Q^{2}\right)=F_{2,0}^{N S}(N)\left[\left(\frac{\alpha_{s}\left(Q_{I}^{2}\right)}{\alpha_{s}\left(Q^{2}\right)}\right)^{\tilde{\Gamma}_{2,0, l}^{N S}(N+1)}+\alpha_{s}\left(Q_{I}^{2}\right) \ln \left(Q_{I}^{2} / A_{2}^{N S}\right) \Gamma_{N S}^{2,0, l}(N+1)\right] .
$$

Of course, this whole discussion of $F_{2}^{N S}\left(N, Q^{2}\right)$ has been rather simplified by the assumption that the higher-order-in- $\alpha_{s}$ terms in the physical anomalous dimension do not contain higher singularities in $(N+1)$. Recognizing that they do, we obtain

$$
\begin{aligned}
& \left(\frac{d F_{2, R S C, 0}^{N S}\left(N, Q^{2}\right)}{d \ln Q^{2}}\right)_{0}=\alpha_{s}\left(Q_{I}^{2}\right) F_{2,0}^{N S}(N)\left(\Gamma_{2,0, l}^{N S}(N+1)+\frac{1}{N+1} \times\right. \\
& \left(\sum_{m=1}^{\infty}\left(\tilde{b}_{m}+(N+1) \tilde{c}_{m}\right)\left(\frac{\alpha_{s}\left(Q_{I}^{2}\right)}{(N+1)^{2}}\right)^{m}+\frac{\tilde{a}_{-1} \alpha_{s}\left(Q_{I}^{2}\right)}{(N+1)} \ln \left(Q_{I}^{2} / A_{2}^{N S}\right) \sum_{m=0}^{\infty} \tilde{b}_{m}\left(\frac{\alpha_{s}\left(Q_{I}^{2}\right)}{(N+1)^{2}}\right)^{m}\right) \times \\
& \left(\frac{\alpha_{s}\left(Q_{I}^{2}\right)}{\alpha_{s}\left(Q^{2}\right)}\right)^{\tilde{\Gamma}_{2,0, l}^{N S}(N+1)-1} \exp \left[\int_{\alpha_{s}\left(Q^{2}\right)}^{\alpha_{s}\left(Q_{I}^{2}\right)}\left(\sum_{m=1}^{\infty}\left(\frac{1}{N+1} \tilde{b}_{m}+\tilde{c}_{m}\right)\left(\frac{\alpha_{s}\left(q^{2}\right)}{(N+1)^{2}}\right)^{m}\right) \frac{d \alpha_{s}\left(q^{2}\right)}{b_{0} \alpha_{s}^{2}\left(q^{2}\right)}\right] \times \\
& \left.\left(\frac{1+\sum_{m=1}^{\infty} \tilde{b}_{m}\left(\alpha_{s}^{m+1}\left(Q^{2}\right) /(N+1)^{2 m}\right)}{1+\sum_{m=1}^{\infty} \tilde{b}_{m}\left(\alpha_{s}^{m+1}\left(Q_{I}^{2}\right) /(N+1)^{2 m}\right)}\right)\right)
\end{aligned}
$$

and

$$
\begin{aligned}
F_{2, R S C, 0}^{N S}\left(N, Q_{I}^{2}\right) & =F_{2,0}^{N S}(N)\left(1+\ln \left(Q_{I}^{2} / A_{2}^{N S}\right) \alpha_{s}\left(Q_{I}^{2}\right)\left(\Gamma_{2,0, l}^{N S}(N+1)+\right.\right. \\
& \left.\left.\frac{1}{N+1} \sum_{m=1}^{\infty} \tilde{b}_{m}\left(\frac{\alpha_{s}\left(Q_{I}^{2}\right)}{(N+1)^{2}}\right)^{m}+\sum_{m=0}^{\infty}\left(\tilde{c}_{m}+\frac{1}{2} \sum_{n=0}^{m} \tilde{b}_{n} \tilde{b}_{m-n} \ln \left(Q_{I}^{2} / A_{2}^{N S}\right)\right)\left(\frac{\alpha_{s}\left(Q_{I}^{2}\right)}{(N+1)^{2}}\right)^{m}\right)\right),
\end{aligned}
$$

where for $F_{2}^{N S}\left(N, Q^{2}\right)$ the coefficients in the series in $(N+1)^{-1}$ are not necessarily the same as for $F_{L}^{N S}\left(N, Q^{2}\right)$, hence the slightly different notation. However, $a_{-1}=\tilde{a}_{-1}$ and $b_{m}=\tilde{b}_{m}$ (probably [39]). The $c_{m}$ are not equal to the $\tilde{c}_{m}$ though, so there is no guarantee that $F_{2, R S C, 0}^{N S}\left(x, Q^{2}\right)$ and $F_{L, R S C, 0}^{N S}\left(x, Q^{2}\right)$ will behave in the same way in the small- $x$ limit.

When all the $b_{m}$ and $c_{m}$ are known they can be used to present an argument for the form of the small- $x$ behaviour of nonsinglet structure functions. Until this happens our discussion of the LORSC calculation of the nonsinglet structure functions is rather academic. However, it has enabled us to discuss many of the issues in a simpler framework than if we had gone directly to the singlet structure functions. We will discuss these singlet structure functions next. 


\subsection{The Renormalization-Scheme-Consistent Singlet Structure Functions.}

When calculating the singlet structure functions we cannot just construct the complete LO evolution and input and combine these to obtain the LO expression. Each of the component parts of the LO expressions for $F_{2}\left(N, Q^{2}\right)$ and $F_{L}\left(N, Q^{2}\right)$ (we omit the superscript $S$ in this section) must consist of LO input parts and evolution parts, but it is not obvious what these are. In order to find the full LORSC expressions for the singlet structure functions we will have to work in steps. We will consider only the full LO expression with the perturbative factors expanded about the particular value of $N=0$, and the simplest way to proceed is to solve the evolution equations in terms of physical anomalous dimensions and structure functions. We have already proved that in the loop expansion the LO expressions only depend on the one-loop physical anomalous dimensions, and in the leading- $\ln (1 / x)$ expansion the LO expressions depend only on (3.7). Hence, it is only the combination of these anomalous dimensions which is considered in our solution.

We cannot simply write the physical anomalous dimension matrix

$$
\left(\begin{array}{cc}
\alpha_{s}\left(Q^{2}\right) \Gamma_{L L}^{0, l}(N)+\Gamma_{L L}^{\tilde{0}}\left(\alpha_{s}\left(Q^{2}\right) / N\right) & \alpha_{s}\left(Q^{2}\right) \Gamma_{L 2}^{0, l}(N)+\Gamma_{L 2}^{\tilde{0}}\left(\alpha_{s}\left(Q^{2}\right) / N\right) \\
\alpha_{s}\left(Q^{2}\right) \Gamma_{2 L}^{0, l}(N)+\alpha_{s}\left(Q^{2}\right) \Gamma_{2 L}^{\tilde{1}}\left(\alpha_{s}\left(Q^{2}\right) / N\right) & \alpha_{s}\left(Q^{2}\right) \Gamma_{22}^{0, l}(N)+\alpha_{s}\left(Q^{2}\right) \Gamma_{22}^{\tilde{1}}\left(\alpha_{s}\left(Q^{2}\right) / N\right)
\end{array}\right)
$$

(where $\Gamma_{L L}^{\tilde{0}}\left(\alpha_{s}\left(Q^{2}\right) / N\right)=\Gamma_{L L}^{0}\left(\alpha_{s}\left(Q^{2}\right) / N\right)$ with the one-loop component subtracted out, etc.), and solve the renormalization group equations because the full solution contains terms which are not properly of leading order. We must choose some way of solving for the structure functions systematically which enables us to extract the true LO behaviour in as simple a manner as possible. In order to do this we take account of the fact that the one-loop solutions for $F_{L}\left(N, Q^{2}\right)$ and $\left(d F_{2}\left(N, Q^{2}\right) / d \ln Q^{2}\right)$ must be part of the complete LORSC solutions. Hence, we split our anomalous dimension matrix up into the form

$$
\alpha_{s}\left(Q^{2}\right)\left(\begin{array}{cc}
\Gamma_{L L}^{0, l}(N)+ & \Gamma_{L 2}^{0, l}(N) \\
\Gamma_{2 L}^{0, l}(N) & \Gamma_{22}^{0, l}(N)
\end{array}\right)+\left(\begin{array}{cc}
\Gamma_{L L}^{\tilde{0}}\left(\alpha_{s}\left(Q^{2}\right) / N\right) & \Gamma_{L 2}^{\tilde{0}}\left(\alpha_{s}\left(Q^{2}\right) / N\right) \\
\alpha_{s}\left(Q^{2}\right) \Gamma_{2 L}^{\tilde{1}}\left(\alpha_{s}\left(Q^{2}\right) / N\right) & \alpha_{s}\left(Q^{2}\right) \Gamma_{22}^{\tilde{1}}\left(\alpha_{s}\left(Q^{2}\right) / N\right)
\end{array}\right),
$$

and solve by treating the second matrix as a perturbation to the first. Doing this we obtain the one-loop solutions as the lowest-order solutions and can systematically calculate corrections to this, extracting the parts of these "corrections" which are leading order.

So, first let us consider the solution to the $\mathcal{O}\left(\alpha_{s}\right)$ renormalization group equation with boundary

conditions $\hat{F}_{L}^{0, l}\left(N, Q_{I}^{2}\right)=\hat{F}_{L}(N)$ and $F_{2}^{0, l}\left(N, Q_{I}^{2}\right)=F_{2}(N)$. We may write the solution for the longitudinal structure function as

$$
\hat{F}_{L}^{0, l}\left(N, Q^{2}\right)=\hat{F}_{L}^{0, l,+}(N)\left(\frac{\alpha_{s}\left(Q_{I}^{2}\right)}{\alpha_{s}\left(Q^{2}\right)}\right)^{\tilde{\Gamma}^{0, l,+}(N)}+\hat{F}_{L}^{0, l,-}(N)\left(\frac{\alpha_{s}\left(Q_{I}^{2}\right)}{\alpha_{s}\left(Q^{2}\right)}\right)^{\tilde{\Gamma}^{0, l,-}(N)},
$$

where $\tilde{\Gamma}^{0, l,+,-}(N)$ are the two eigenvalues of the zeroth-order physical anomalous dimension matrix (which are the same as the eigenvalues of the zeroth-order parton anomalous dimension matrix), and in practice

$$
\hat{F}_{L}^{0, l,+}(N)=\hat{F}_{L}(N)-\frac{36-8 N_{f}}{27} F_{2}(N)+\mathcal{O}(N), \quad \hat{F}_{L}^{0, l,-}(N)=\frac{36-8 N_{f}}{27} F_{2}(N)+\mathcal{O}(N) .
$$


Having chosen to write the lowest-order solution for the longitudinal structure function in this way we may then write the lowest-order solution for $F_{2}\left(N, Q^{2}\right)$ as

$$
\hat{F}_{2}^{0, l}\left(N, Q^{2}\right)=e^{+}(N) \hat{F}_{L}^{0, l,+}(N)\left(\frac{\alpha_{s}\left(Q_{I}^{2}\right)}{\alpha_{s}\left(Q^{2}\right)}\right)^{\tilde{\Gamma}^{0, l,+}(N)}+e^{-}(N) \hat{F}_{L}^{0, l,-}(N)\left(\frac{\alpha_{s}\left(Q_{I}^{2}\right)}{\alpha_{s}\left(Q^{2}\right)}\right)^{\tilde{\Gamma}^{0, l,-}(N)}
$$

where

$$
\begin{aligned}
& e^{+}(N)=\left(\frac{\Gamma^{0, l,+}(N)-\Gamma_{L L}^{0, l}(N)}{\Gamma_{L 2}^{0, l}(N)}\right)=N / 6+\mathcal{O}\left(N^{2}\right), \\
& e^{-}(N)=\left(\frac{\Gamma^{0, l,-}(N)-\Gamma_{L L}^{0, l}(N)}{\Gamma_{L 2}^{0, l}(N)}\right)=\left(\frac{27}{36-8 N_{f}}\right)+\mathcal{O}(N),
\end{aligned}
$$

The first corrections to the one-loop solution may be obtained by solving the equations,

$$
\begin{aligned}
& \frac{d}{d \ln Q^{2}}\left(\begin{array}{c}
\hat{F}_{L}^{c 1}\left(N, Q^{2}\right) \\
F_{2}^{c 1}\left(N, Q^{2}\right)
\end{array}\right)=\alpha_{s}\left(Q^{2}\right)\left(\begin{array}{cc}
\Gamma_{L L}^{0, l}(N) & \Gamma_{L 2}^{0, l}(N) \\
\Gamma_{2 L}^{0, l}(N) & \Gamma_{22}^{0, l}(N)
\end{array}\right)\left(\begin{array}{l}
\hat{F}_{L}^{c 1}\left(N, Q^{2}\right) \\
F_{2}^{c 1}\left(N, Q^{2}\right)
\end{array}\right) \\
& +\left(\begin{array}{cc}
\Gamma_{L L}^{\tilde{0}}\left(\alpha_{s}\left(Q^{2}\right) / N\right) & -\frac{\left(36-8 N_{f}\right)}{27} \Gamma_{L L}^{\tilde{0}}\left(\alpha_{s}\left(Q^{2}\right) / N\right) \\
\alpha_{s}\left(Q^{2}\right) \Gamma_{2 L}^{\tilde{1}}\left(\alpha_{s}\left(Q^{2}\right) / N\right) & -\frac{\left(36-8 N_{f}\right)}{27} \alpha_{s}\left(Q^{2}\right) \Gamma_{2 L}^{\tilde{1}}\left(\alpha_{s}\left(Q^{2}\right) / N\right)
\end{array}\right)\left(\begin{array}{l}
\hat{F}_{L}^{0, l}\left(N, Q^{2}\right) \\
F_{2}^{0, l}\left(N, Q^{2}\right)
\end{array}\right),
\end{aligned}
$$

where we have used the relationships between the physical anomalous dimensions in order to simplify the second matrix. We proceed as follows. First we define the vectors

$$
\underline{e}^{+}(N)=\left(\begin{array}{c}
1 \\
e^{+}(N)
\end{array}\right), \quad \underline{e}^{-}(N)=\left(\begin{array}{c}
1 \\
e^{-}(N)
\end{array}\right), \quad \underline{F}^{c 1}\left(N, Q^{2}\right)=\left(\begin{array}{c}
F_{L}^{c 1}\left(N, Q^{2}\right) \\
F_{2}^{c 1}\left(N, Q^{2}\right)
\end{array}\right),
$$

and write

$$
\underline{F}^{c 1}\left(N, Q^{2}\right)=\underline{e}^{+}(N) F^{c 1,+}\left(N, Q^{2}\right)+\underline{e}^{-}(N) F^{c 1,-}\left(N, Q^{2}\right) .
$$

We also define projection operators $\underline{p}^{+}(N)$ and $\underline{p}^{-}(N)\left(\underline{p}^{+}(N) \cdot \underline{e}^{+}(N)=1, \underline{p}^{+}(N) \cdot \underline{e}^{-}(N)=0\right.$ etc.) which in practice are

$$
\underline{p}^{+}(N)=\left(\begin{array}{c}
1 \\
\frac{8 N_{f}-36}{27}
\end{array}\right)+\mathcal{O}(N), \quad \underline{p}^{-}(N)=\left(\begin{array}{c}
0 \\
\frac{27}{8 N_{f}-36}
\end{array}\right)+\mathcal{O}(N) .
$$

Multiplying (4.97) by $\underline{p}^{+}(N)$ or $p^{-}(N)$ leads to straightforward first-order differential equations for $F^{c 1,+}\left(N, Q^{2}\right)$ or $F^{c 1,-}\left(N, Q^{2}\right)$. Writing the zeroth-order solution $\underline{F}^{0, l}\left(N, Q^{2}\right)$, in the form

$$
\underline{F}^{0, l}\left(N, Q^{2}\right)=\underline{e}^{+}(N) F^{0, l,+}\left(N, Q^{2}\right)+\underline{e}^{-}(N) F^{0, l,-}\left(N, Q^{2}\right)
$$

these equation can now be solved by using the power series expansions of $\underline{e}^{+(-)}(N)$ and $\underline{p}^{+(-)}(N)$ in terms of $N$. Only a small part of the overall solution contributes at LO. 
Solving for the complete leading-order part of $F^{+}\left(N, Q^{2}\right)$ inductively in this manner, and making it formally insensitive to changes of $Q_{I}^{2}$ we get

$$
\begin{gathered}
F_{R S C, 0}^{+}\left(N, Q^{2}\right)=\left[\left(\hat{F}_{L}(N)-\left(\frac{36-8 N_{f}}{27}\right) F_{2}(N)\right)\left(\exp \left[\ln \left(Q_{I}^{2} / A_{L L}\right) \Gamma_{L L}^{0}\left(\alpha_{s}\left(Q_{I}^{2}\right) / N\right)\right]-1\right)\right. \\
\left.+\hat{F}_{L}^{0, l,+}(N)\right]\left(\frac{\alpha_{s}\left(Q_{I}^{2}\right)}{\alpha_{s}\left(Q^{2}\right)}\right)^{\tilde{\Gamma}^{0, l,+}(N)} \exp \left[\int_{\alpha_{s}\left(Q^{2}\right)}^{\alpha_{s}\left(Q_{I}^{2}\right)} \frac{\tilde{\Gamma}_{L L}^{\tilde{0}}\left(\alpha_{s}\left(q^{2}\right) / N\right)}{\alpha_{s}^{2}\left(q^{2}\right)} d \alpha_{s}\left(q^{2}\right)\right] .
\end{gathered}
$$

We can solve for the corrections which are proportional to $\underline{e}^{-}(N)$ in exactly the same manner, using $\underline{p}^{-}(N)$ instead of $\underline{p}^{+}(N)$. Inserting the whole of $F_{R S C, 0}^{+}\left(N, Q^{2}\right)$ into the right-hand side the solution is relatively simple if we wish to keep only the LO parts. Adding to the solution at one-loop, the whole of the LO part of $F^{-}\left(N, Q^{2}\right)$ is

$$
\begin{aligned}
& F_{R S C, 0}^{-}\left(N, Q^{2}\right)=\left(F_{L}^{0, l,-}(N)-\left(\frac{36-8 N_{f}}{27}\right)\left(\hat{F}_{L}(N)-\left(\frac{36-8 N_{f}}{27}\right) F_{2}(N)\right) \times\right. \\
& \left(\frac{\alpha_{s}\left(Q_{I}^{2}\right) \Gamma_{2 L}^{\tilde{1}}\left(\alpha_{s}\left(Q_{I}^{2}\right) / N\right)-\frac{N}{6} \Gamma_{L L}^{\tilde{0}}\left(\alpha_{s}\left(Q_{I}^{2}\right) / N\right)}{\Gamma_{L L}^{0}\left(\alpha_{s}\left(Q_{I}^{2}\right) / N\right)}\right)\left(\frac{\alpha_{s}\left(Q_{I}^{2}\right)}{\alpha_{s}\left(Q^{2}\right)}\right)^{\tilde{\Gamma}^{0, l,-}(N)}+ \\
& \left(\frac{36-8 N_{f}}{27}\right)\left(\frac{\alpha_{s}\left(Q_{I}^{2}\right) \Gamma_{2 L}^{\tilde{1}}\left(\alpha_{s}\left(Q_{I}^{2}\right) / N\right)-\frac{N}{6} \Gamma_{L L}^{\tilde{0}}\left(\alpha_{s}\left(Q_{I}^{2}\right) / N\right)}{\Gamma_{L L}^{0}\left(\alpha_{s}\left(Q_{I}^{2}\right) / N\right)}\right)\left(\hat{F}_{L}(N)-\left(\frac{36-8 N_{f}}{27}\right) F_{2}(N)\right) \times \\
& \exp \left[\ln \left(Q_{I}^{2} / A_{L L}\right) \Gamma_{L L}^{0}\left(\alpha_{s}\left(Q_{I}^{2}\right) / N\right)\right]\left(\frac{\alpha_{s}\left(Q_{I}^{2}\right)}{\alpha_{s}\left(Q^{2}\right)}\right)^{\tilde{\Gamma}^{0, l,+}(N)} \exp \left[\int_{\alpha_{s}\left(Q^{2}\right)}^{\alpha_{s}\left(Q_{I}^{2}\right)} \frac{\tilde{\Gamma}_{L L}^{\tilde{0}}\left(\alpha_{s}\left(q^{2}\right) / N\right)}{\alpha_{s}^{2}\left(q^{2}\right)} d \alpha_{s}\left(q^{2}\right)\right] \\
& + \text { higher order, }
\end{aligned}
$$

where, in order to make the expression invariant under changes in starting scale, and also ensure that for $Q_{I}^{2}=A_{L L}$ we have $F^{-}\left(N, Q_{I}^{2}\right)=\hat{F}_{L}^{0, l,-}(N)$, we make the choice

$$
\begin{aligned}
F^{\text {cfull },-}\left(N, Q_{I}^{2}\right)= & \left(\frac{36-8 N_{f}}{27}\right)\left(\frac{\alpha_{s}\left(Q_{I}^{2}\right) \Gamma_{2 L}^{\tilde{1}}\left(\alpha_{s}\left(Q_{I}^{2}\right) / N\right)-\frac{N}{6} \Gamma_{L L}^{\tilde{0}}\left(\alpha_{s}\left(Q_{I}^{2}\right) / N\right)}{\Gamma_{L L}^{0}\left(\alpha_{s}\left(Q_{I}^{2}\right) / N\right)}\right) \times \\
& \left(\hat{F}_{L}(N)-\left(\frac{36-8 N_{f}}{27}\right) F_{2}(N)\right)\left(\exp \left[\ln \left(Q_{I}^{2} / A_{L L}\right) \Gamma_{L L}^{0}\left(\alpha_{s}\left(Q_{I}^{2}\right) / N\right)\right]-1\right) .
\end{aligned}
$$

We must now use the leading parts of $F^{+}\left(N, Q^{2}\right)$ and $F^{-}\left(N, Q^{2}\right)$ in order to obtain LORSC expressions for the structure functions. The way in which we have set up the calculation makes this very straightforward for the longitudinal structure function: we multiply $F_{R S C, 0}^{+}\left(N, Q^{2}\right)$ and $F_{R S C, 0}^{-}\left(N, Q^{2}\right)$ by $\left(\alpha_{s}\left(Q^{2}\right) / 2 \pi\right)$, where the only part of $F_{R S C, 0}^{-}\left(N, Q^{2}\right)$ which contributes to the 
LORSC expression for $F_{L}\left(N, Q^{2}\right)$ is the one-loop part,

$$
\begin{aligned}
F_{L, R S C, 0}(N, & \left.Q^{2}\right)=\frac{\alpha_{s}\left(Q_{I}^{2}\right)}{2 \pi}\left[\left(\frac{\alpha_{s}\left(Q_{I}^{2}\right)}{\alpha_{s}\left(Q^{2}\right)}\right)^{\tilde{\Gamma}^{0, l,+}(N)-1} \exp \left[\int_{\alpha_{s}\left(Q^{2}\right)}^{\alpha_{s}\left(Q_{I}^{2}\right)} \frac{\tilde{\Gamma}_{L L}^{0}\left(\alpha_{s}\left(q^{2}\right) / N\right)}{\alpha_{s}\left(q^{2}\right)} d \alpha_{s}\left(q^{2}\right)\right] \times\right. \\
& \left(F_{L}^{0, l,+}(N)+\left(\hat{F}_{L}(N)-\left(\frac{36-8 N_{f}}{27}\right) F_{2}(N)\right)\left(\exp \left[\ln \left(Q_{I}^{2} / A_{L L}\right) \Gamma_{L L}^{0}\left(\alpha_{s}\left(Q_{I}^{2}\right) / N\right)\right]-1\right)\right) \\
& \left.+F_{L}^{0, l,-}(N)\left(\frac{\alpha_{s}\left(Q_{I}^{2}\right)}{\alpha_{s}\left(Q^{2}\right)}\right)^{\tilde{\Gamma}^{0, l,-}(N)-1}\right] .
\end{aligned}
$$

We also wish to find the LORSC expressions for $\left(d F_{2}\left(N, Q^{2}\right) / d \ln Q^{2}\right)$ and for the input $F_{2}\left(N, Q_{I}^{2}\right)$. We consider the former first. Using the form of $e^{+}(N)$ and $e^{-}(N)$ in (4.96) it is clear that, besides for the one-loop contributions, the LORSC expression will come from $(27 /(36-$ $\left.\left.8 N_{f}\right)\right) \times\left(d F_{2}^{-}\left(N, Q^{2}\right) / d \ln Q^{2}\right)$ and from $N / 6 \times\left(d F_{2}^{+}\left(N, Q^{2}\right) / d \ln Q^{2}\right)$. Explicitly we obtain

$$
\begin{aligned}
& \left(\frac{d F_{2}\left(N, Q^{2}\right)}{d \ln Q^{2}}\right)_{R S C, 0}=\alpha_{s}\left(Q_{I}^{2}\right)\left[e^{-}(N) \Gamma^{0, l,-}(N) \hat{F}_{L}^{0, l,-}(N)\left(\frac{\alpha_{s}\left(Q_{I}^{2}\right)}{\alpha_{s}\left(Q^{2}\right)}\right)^{\tilde{\Gamma}^{0, l,-}(N)-1}\right. \\
& +\left(e^{+}(N) \Gamma^{0, l,+}(N) \hat{F}_{L}^{0, l,+}(N)-\Gamma_{2, L}^{1,0}(N)\left(\hat{F}_{L}(N)-\left(\frac{36-8 N_{f}}{27}\right) F_{2}(N)\right)\right. \\
& \left.+\Gamma_{2 L}^{1}\left(\alpha_{s}\left(Q_{I}^{2}\right) / N\right)\left(\hat{F}_{L}(N)-\left(\frac{36-8 N_{f}}{27}\right) F_{2}(N)\right) \exp \left[\ln \left(Q_{I}^{2} / A_{L L}\right) \Gamma_{L L}^{0}\left(\alpha_{s}\left(Q_{I}^{2}\right) / N\right)\right]\right) \times \\
& \left.\exp \left[\int_{\alpha_{s}\left(Q^{2}\right)}^{\alpha_{s}\left(Q_{I}^{2}\right)} \frac{\tilde{\Gamma}_{L L}^{\tilde{0}}\left(\alpha_{s}\left(q^{2}\right) / N\right)}{\alpha_{s}^{2}\left(q^{2}\right)} d \alpha_{s}\left(q^{2}\right)\right]\left(\frac{\alpha_{s}\left(Q_{I}^{2}\right)}{\alpha_{s}\left(Q^{2}\right)}\right)^{\tilde{\Gamma}^{0, l,+}(N)-1}\right],
\end{aligned}
$$

where $\frac{N}{6}\left(\Gamma_{L L}^{0}\left(\alpha_{s}\left(Q^{2}\right) / N\right)-\Gamma_{L L}^{\tilde{0}}\left(\alpha_{s}\left(Q^{2}\right) / N\right)\right)=\alpha_{s}\left(Q^{2}\right)\left(\Gamma_{2 L}^{1}\left(\alpha_{s}\left(Q^{2}\right) / N\right)-\Gamma_{2 L}^{\tilde{1}}\left(\alpha_{s}\left(Q^{2}\right) / N\right)\right)$ has been used and $\Gamma_{2, L}^{1,0}(N)\left(\hat{F}_{L}(N)-\left(\frac{36-8 N_{f}}{27}\right) F_{2}(N)\right)$ is the part of the input which is common to both the one-loop and the leading- $\ln (1 / x)$ input, and is subtracted from the former in order to avoid double counting. Similarly the input for $F_{2}\left(N, Q^{2}\right)$ is given by the relatively simple form

$$
\begin{aligned}
& F_{2, R S C, 0}\left(N, Q_{I}^{2}\right)=F_{2}(N) \\
& +\alpha_{s}\left(Q_{I}^{2}\right) \frac{\Gamma_{2 L}^{1}\left(\alpha_{s}\left(Q_{I}^{2}\right) / N\right)}{\Gamma_{L L}^{0}\left(\alpha_{s}\left(Q_{I}^{2}\right) / N\right)}\left(\hat{F}_{L}(N)-\frac{\left(36-8 N_{f}\right)}{27} F_{2}(N)\right)\left(\exp \left[\ln \left(Q_{I}^{2} / A_{L L}\right) \Gamma_{L L}^{0}\left(\alpha_{s}\left(Q_{I}^{2}\right) / N\right)\right]-1\right) \\
& +\ln \left(Q_{I}^{2} / A_{L L}\right) \alpha_{s}\left(Q_{I}^{2}\right)\left(e^{+}(N) \Gamma^{0, l,+}(N) \hat{F}_{L}^{0, l,+}\left(N, Q_{I}^{2}\right)+e^{-}(N) \Gamma^{0, l,-}(N) \hat{F}_{L}^{0, l,-}\left(N, Q_{I}^{2}\right)\right. \\
& \left.\quad-\Gamma_{2, L}^{1,0}(N)\left(\hat{F}_{L}(N)-\left(\frac{36-8 N_{f}}{27}\right) F_{2}(N)\right)\right) .
\end{aligned}
$$

The third term is the renormalization-scheme-invariant order- $\alpha_{s}\left(Q_{I}^{2}\right)$ input, which must compensate for changes in the one-loop evolution under changes in $Q_{I}^{2}$. Again we explicitly extract a term $\propto \ln \left(Q_{I}^{2} / A\right) \Gamma_{2, L}^{1,0}(N)\left(\hat{F}_{L}(N)-\left(\frac{36-8 N_{f}}{27}\right) F_{2}(N)\right)$ in order to avoid double counting. The fact that 
this term can be thought of as appearing from two different sources leads us to choose both our unknown scale constants in the input equal to the same value $A_{L L}$. We note that our choice of inputs not only ensures $Q_{I}^{2}$-invariance up to higher orders, but are also such that our expressions for $\hat{F}_{L, R S C, 0}\left(N, Q_{I}^{2}\right)$ and $F_{2, R S C, 0}\left(N, Q_{I}^{2}\right)$ reduce to the nonperturbative inputs if $Q_{I}^{2}=A_{L L}$. Having obtained our LORSC expressions for $\left(d F_{2}\left(N, Q^{2}\right) / d \ln Q^{2}\right)$ and $F_{2}\left(N, Q_{I}^{2}\right)$, then as already argued, in order to obtain our expression for $F_{2}\left(N, Q^{2}\right)$ we integrate $\left(d F_{2}\left(N, Q^{2}\right) / d \ln Q^{2}\right)_{R S C, 0}$ from $Q_{I}^{2}$ to $Q^{2}$ and add to the input $F_{2, R S C, 0}\left(N, Q_{I}^{2}\right)$.

Thus, we have our complete leading-order renormalization-scheme-consistent expressions for the structure functions. These are significantly different from both the one-loop expressions and the leading $-\ln (1 / x)$ expressions, although they do reduce to them in the appropriate limits. Indeed, once we include the $\mathcal{O}\left(\alpha_{s}\left(Q_{I}^{2}\right)\right)$ inputs for $F_{2}\left(N, Q^{2}\right)$ in the definition of the leading-order inputs in the loop-expansion (as we should), each of the terms in the inputs and evolution terms in (4.105), (4.106) and (4.107) contains a part which appears in both the LO expression in the loop expansion and the LO expression in the leading $\ln (1 / x)$ expansion. Our full LORSC expressions are obtained rather more easily than above by simply letting each of the input and evolution terms become the combination of the terms in the two expansion schemes. In a sense this result is obvious, but it is necessary to verify this by deriving the expressions as above.

Let us comment on the form of our final LORSC expressions. We note that as for the leading$\ln (1 / x)$ expansion, the LORSC expansion still leads to predictions for all the small- $x$ inputs: predictions of each in terms of the nonperturbative inputs (which we imagine should be quite flat) and the nonperturbative scale $A_{L L}$, and also stronger predictions for the relationships between the inputs (although the scale $Q_{I}^{2}$ at which they should be imposed is not determined).

Finally, we notice that each of the terms appearing in our expressions is manifestly renormalization scheme invariant, and it is clear that no terms are subleading in $\alpha_{s}$ to any other terms, either in the input or in the evolution. If we had simply solved the renormalization group equations using the whole of the anomalous dimension matrix (4.91) then we would have obtained many terms which do not appear in our full leading-order expressions (4.105), (4.106) and (4.107). These would still be renormalization scheme independent, since our input anomalous dimensions are renormalization scheme independent. However, they would be of the same form as terms which are renormalization scheme dependent (e.g. we saw in 4.2 that the subleading-in- $\ln (1 / x)$ evolution $\Phi_{1}^{+}\left(Q^{2}, Q_{I}^{2}\right)$ has a manifestly renormalization-scheme-independent part depending on $\Gamma_{2 L}^{1}\left(\alpha_{s}\left(Q^{2}\right) / N\right)$ as well as a renormalization-scheme-dependent part depending on $\left.\Gamma_{L L}^{1}\left(\alpha_{s}\left(Q^{2}\right) / N\right)\right)$. These terms should be dropped, and (4.105), (4.106) and (4.107) are the correct expressions for the structure functions to be used with the one-loop coupling constant. 


\section{5. $x$-Space Solutions.}

We shall now discuss how we use the expressions (4.105), (4.106) and (4.107) in order to obtain our expressions for the $x$-space structure functions and ultimately compare with data. The data on $\mathcal{F}_{2}\left(x, Q^{2}\right)$ exist over a range of $Q^{2}$ from $\sim 0.2 \mathrm{GeV}^{2}$ to $5000 \mathrm{GeV}^{2}$, though in practice we will impose a lower cut on the data of $Q^{2}=2 \mathrm{GeV}^{2}$, except for the HERA data where we choose $Q^{2}=1.5 \mathrm{GeV}^{2}$ simply in order not to lose some of the very low $x$ data. The threshold of heavy quark production is $W^{2}=4 m_{H}^{2}$, where $W$ is the invariant mass of the hadronic system created from the struck proton (or neutron) and in the limit of zero proton (and/or neutron) mass is given by $W^{2}=Q^{2}\left(x^{-1}-1\right)$. $m_{H}$ is the mass of the heavy quark. Thus, we clearly work in the limit where the up, down and strange quarks are effectively massless. However, we cross the $b$-quark threshold of $W^{2} \approx 20 \mathrm{GeV}^{2}$, and at the lower end of our range are in the region of the $c$-quark threshold of $W^{2} \approx 2 \mathrm{GeV}^{2}$. Hence, we will need expressions for the structure functions which cross quark thresholds.

The correct treatment of these heavy quark thresholds is less well established than the treatment of effectively massless quarks, and is certainly more complicated. Here we use the prescription for treating heavy quarks outlined in [41]. This involves treating all the quarks as massless, but only allowing the heavy quarks to become active above the simple threshold $Q^{2}=m_{H}^{2}$. Hence, the value of $N_{f}$ appearing explicitly in any expressions changes discontinuously at this threshold. The running coupling constant, is defined to be continuous at the thresholds. It is determined by the relationship

$$
\alpha_{s, n}\left(Q^{2}\right)=\alpha_{s, n+1}\left(Q^{2}\right)\left(1+\frac{\alpha_{s, n+1}\left(Q^{2}\right)}{6 \pi} \ln \left(m_{n+1}^{2} / Q^{2}\right)\right)
$$

where the central $\alpha_{s}\left(Q^{2}\right)$ with $N_{f}=4$ is defined by

$$
\alpha_{s, 4}\left(Q^{2}\right)=\frac{12 \pi}{\left(33-2 \cdot\left(N_{f}=4\right)\right) \ln \left(Q^{2} / \Lambda_{Q C D, 4}^{2}\right)} .
$$

This complete prescription for treatment of the heavy quarks is consistent with the decoupling theorem, as it is guaranteed to provide the correct expressions far above or below any threshold: the increase in the coupling below a threshold compensating for the absence of virtual heavy quarks in calculations below this threshold. It is clearly rather unsatisfactory in the region of the threshold, with heavy quark structure functions having an abrupt threshold in $Q^{2}$ rather than a smooth threshold in $W^{2}$. Work to rectify this is in progress, and will certainly involve the use of the heavy quark coefficient functions at leading order in $\ln (1 / x)$ already calculated [13][31].

We can now discuss the form of the $x$-space solutions for the structure functions. It is the perturbative part of the moment-space structure functions for which we can produce well-ordered, RSC expressions and the nonperturbative parts of the expressions for these structure functions, 
$F_{2}(N)$ and $\hat{F}_{L}(N)$, will be nonanalytic, complicated functions of $N$. Our complete moment-space expression for a general structure function (or derivative of a structure function) will be

$$
F_{i}\left(N, Q^{2}\right)=P_{i, 2}\left(N, \alpha_{s}\right) F_{2}(N)+P_{i, L}\left(N, \alpha_{s}\right) \hat{F}_{L}(N),
$$

where the $P_{i j}\left(N, \alpha_{s}\right)$ 's are the calculable perturbative components of the complete expressions which can be expanded as power series in both $\alpha_{s}$ and $N$, i.e. examples of the physically relevant perturbative functions we discussed in $\S 4.3$. We obtain the $x$-space structure functions as follows. We factor out the perturbative part of each of these expressions which is proportional to either $\hat{F}_{L}(N)$ or $F_{2}(N)$, and take the inverse Mellin transformation of this perturbative function by integrating around a contour encircling $N=0$, obtaining the leading-in- $\ln (1 / x)$, at lowest-order-in- $x$ parts of the perturbative $\mathcal{P}\left(x, \alpha_{s}\right)$ 's as well as all the lowest-order-in- $\alpha_{s}$ parts at lowest-orderin $-x$. In order to obtain the leading-order-in- $\ln (1 / x)-$ and $-\alpha_{s}$, at lowest-order-in- $x$, part of the perturbatively calculated structure functions we then convolute with the whole of the nonperturbative $\hat{F}_{L}(x)$ and $F_{2}(x)$ which are obtained by a complete inverse Mellin transformation of $\hat{F}_{L}(N)$ and $F_{2}(N)$. In principle, the full LORSC $x$-space structure functions are calculated by repeating this procedure for the LORSC moment-space structure functions where the perturbative parts are expanded about $N$ equal to all negative integers, but in practice one only really needs include the one-loop, or leading-in- $\alpha_{s}$ part of the full LORSC perturbative parts for all negative integers.

In practice the calculation of the structure functions is performed in a rather different manner. The structure functions are calculated using a modification of the computer program that is used by Martin, Roberts and Stirling in their global fits to structure function data. This works in terms of parton densities directly in $x$-space. Input parton densities are specified at some scale $Q_{0}^{2}$ and the $Q^{2}$ evolution is calculated on a grid in $x$ and $Q^{2}$. This evolution is obtained by integrating up the renormalization group equations involving the complete parton distributions and full specified splitting functions. The structure functions are calculated by numerically performing the convolutions of the resulting $Q^{2}$-dependent parton distributions with coefficient functions. Thus, in fact, we obtain the scheme-independent LORSC structure functions by working in terms of parton densities and choosing coefficient functions and splitting functions (i.e. an effective factorization scheme) which will reproduce the correct expressions as closely as possible.

We will briefly describe our choice of effective splitting functions as follows. At first order in $\alpha_{s}$ we choose the normal parton splitting functions. We then add corrections to these in order to reproduce our desired results. Denoting these corrections by $\Delta \gamma_{a b}\left(N, \alpha_{s}\left(Q^{2}\right)\right)$, for a given parton to parton splitting function, and expressing these in moment space and in terms of previously discussed quantities for simplicity we get for $\mathcal{F}_{L}\left(x, Q^{2}\right)$

$$
\begin{aligned}
& \Delta \gamma_{f g}\left(N, \alpha_{s}\left(Q^{2}\right)\right)=\Delta \gamma_{f f}\left(N, \alpha_{s}\left(Q^{2}\right)\right)=0 \\
& \Delta \gamma_{g g}\left(N, \alpha_{s}\left(Q^{2}\right)\right)=\frac{9}{4} \Delta \gamma_{g f}\left(N, \alpha_{s}\left(Q^{2}\right)\right)=\Gamma_{L L}^{\tilde{0}}\left(\alpha_{s}\left(Q^{2}\right) / N\right) .
\end{aligned}
$$


This form is not too difficult to understand by looking at e.g. (4.105): the only leading- $\ln (1 / x)$ enhancement of the one-loop expression comes from the evolution in the longitudinal sector which is directly related to the enhanced gluon evolution and hence corrections to the gluon anomalous dimensions. For $\mathcal{F}_{2}\left(x, Q^{2}\right)$ the corrections are a little more involved:

$$
\begin{aligned}
& \Delta \gamma_{f g}\left(N, \alpha_{s}\left(Q^{2}\right)\right)=\alpha_{s}\left(Q^{2}\right) \hat{C}_{2,1, l}^{g}(N) \Gamma_{2 L}^{\tilde{1}}\left(\alpha_{s}\left(Q_{I}^{2}\right) / N\right) \\
& \Delta \gamma_{f f}\left(N, \alpha_{s}\left(Q^{2}\right)\right)=\alpha_{s}\left(Q^{2}\right)\left[\left(\frac{8 N_{f}}{27}-\frac{4}{3}\right)+\frac{2}{N_{f}} \hat{C}_{2,1, l}^{g}(N)\right] \Gamma_{2 L}^{\tilde{1}}\left(\alpha_{s}\left(Q_{I}^{2}\right) / N\right) \\
& \Delta \gamma_{g g}\left(N, \alpha_{s}\left(Q^{2}\right)\right)=\Gamma_{L L}^{\tilde{0}}\left(\alpha_{s}\left(Q^{2}\right) / N\right)-\frac{4}{9} \Delta \gamma_{f g}\left(N, \alpha_{s}\left(Q^{2}\right)\right) \\
& \Delta \gamma_{g f}\left(N, \alpha_{s}\left(Q^{2}\right)\right)=\frac{4}{9} \Gamma_{L L}^{\tilde{0}}\left(\alpha_{s}\left(Q^{2}\right) / N\right)-\frac{4}{9} \Delta \gamma_{f f}\left(N, \alpha_{s}\left(Q^{2}\right)\right)
\end{aligned}
$$

The corrections to the quark anomalous dimensions are functions of $Q_{I}^{2}$ rather than $Q^{2}$, except for the single power of $\alpha_{s}\left(Q^{2}\right)$, because $\Gamma_{2 L}^{1}\left(\alpha_{s} / N\right)$ appears only in the input terms in (4.106). In the small- $x$, or small- $N$ limit we have the simplifications that $\Delta \gamma_{f g}\left(N, \alpha_{s}\left(Q^{2}\right)\right) \rightarrow$ $\alpha_{s}\left(Q^{2}\right)\left(2 N_{f} / 3\right) \Gamma_{2 L}^{\tilde{1}}\left(\alpha_{s}\left(Q_{I}^{2}\right) / N\right)$ and $\Delta \gamma_{f f}\left(N, \alpha_{s}\left(Q^{2}\right)\right) \rightarrow \frac{4}{9} \Delta \gamma_{f g}\left(N, \alpha_{s}\left(Q^{2}\right)\right)$. Thus, in this limit these corrections take on the standard form of $\alpha_{s}\left(Q^{2}\right) \gamma_{f g}^{1}\left(\alpha_{s}\left(Q_{I}^{2}\right) / N\right)$ and $\alpha_{s}\left(Q^{2}\right) \gamma_{f f}^{1}\left(\alpha_{s}\left(Q_{I}^{2}\right) / N\right)$ minus their one-loop components. The prefactors multiplying these terms, which depend on the one-loop longitudinal coefficient functions, ensure that the leading $-\ln (1 / x)$ enhancement of the rate of growth of $\mathcal{F}_{2}\left(x, Q^{2}\right)$ is directly coupled to the longitudinal structure, not to the gluon. This delays the enhancement to slightly smaller values of $x$, and reduces it a little. The contributions to the gluon anomalous dimensions beyond LO in $\ln (1 / x)$ are present to counter the exponentiation of the corrections to the quark anomalous dimensions.

We perform checks to see if the particular choice above does indeed lead to an accurate representation of the correct expressions. In order to do this we use the types of techniques outlined in [22] to transform the exact expressions (4.105), (4.106) and (4.107) to $x$-space obtaining analytic solutions for the structure functions. These expressions are very complicated, but are almost exact for very low values of $x$, and are compared, with the calculations performed using the computer program in $x$-space. For $x \lesssim 0.1$ our choice of the anomalous dimensions leads to agreement with the analytic expressions to an accuracy much better than the errors on the data in any appropriate range of parameter space. At higher $x$ setting to zero all terms in the MRS program other than those coming from one loop, the expressions for the structure functions for $x>0.1$, are very close to the one-loop expressions alone, which agrees with calculations taking the inverse Mellin transformation of the LORSC moment-space expressions. Therefore, the correct choice of effective splitting functions leads to the MRS program producing a very accurate approximation to our correct LORSC expressions for the structure functions over the complete range of parameter space.

Now that it has been determined that our choice of splitting functions is correct, one can vary the input parameters in the standard way to obtain the best fit. In practice the inputs are of the 
standard MRS form (4.24), in terms of partons, but these are constrained by demanding that the resulting structure functions at $Q_{I}^{2}$ are compatible with forms in (4.105), (4.106) and (4.107)(for some $\left.A_{L L}\right)$ where the nonperturbative inputs are flat at small $x$, i.e. they must be described well by a function of the form

$$
\mathcal{F}_{i}(x)=F_{i}(1-x)^{\eta_{i}}\left(1+\epsilon_{i} x^{0.5}+\gamma_{i} x\right) .
$$

We note that with our choice of splitting functions and definitions of parton densities then momentum is not conserved by the evolution: in the best fit to $\mathcal{F}_{2}\left(x, Q^{2}\right)$ discussed in the next section the total momentum carried by the partons at $Q^{2}=2 \mathrm{GeV}^{2}$ is $87 \%$ and at $Q^{2}=5000 \mathrm{GeV}^{2}$ it is about $94 \%$. Hence, the amount of momentum violation is at the level of a few percent. We have already defended this violation of momentum conservation in subsection 4.3. We now also point out that starting with our definition of the partons we could define new parton densities and splitting functions by defining non-zero $C_{2,1}^{f(g)}\left(\alpha_{s} / N\right)$ and $C_{L, 1}^{f(g)}\left(\alpha_{s} / N\right)$ beyond one-loop, and use the transformation rules in $\S 2$ to keep the structure functions unchanged. If these coefficient functions had negative coefficients then, compared to our prescription above, the low $Q^{2}$ parton distributions would need to be larger, and would hence carry more momentum. As $Q^{2}$ increased the effect of these new coefficient functions would decrease, and the extra amount of momentum carried by the new parton distributions compared to the original ones would decrease. Thus, the effect of such a redefinition of parton distributions would be to increase the amount of momentum carried by the partons at low $Q^{2}$ and also to slow the growth of momentum with $Q^{2}$ (or even to turn it into a fall). With a judicious redefinition of coefficient functions of this sort (with perhaps some dependence on $\alpha_{s}\left(Q_{I}^{2}\right)$ as well as $\left.\alpha_{s}\left(Q^{2}\right)\right)$ it should clearly be possible to find an effective factorization scheme where the momentum violation will be extremely small, and one may think of this as a "physical scheme". We have not seriously investigated this redefinition of parton densities in any quantitative manner since it will not affect any physical quantities.

Having specified precisely how we will perform our calculations of structure functions we can make some comment on the general form the structure functions have to take. The expressions for $\mathcal{F}_{L}\left(x, Q^{2}\right)$ and $\mathcal{F}_{2}\left(x, Q^{2}\right)$ depend on $\Gamma_{L L}^{0}\left(\alpha_{s} / N\right)$ and $\Gamma_{2 L}^{1}\left(\alpha_{s} / N\right)$. Both of these series have coefficients which behave like $n^{-3 / 2}(12 \ln 2) / \pi$ for very large $n$. This leads to a cut in the $N-$ plane at $N=\bar{\alpha}_{s} 4 \ln 2$ in both cases, and to the structure functions having asymptotic behaviour $\mathcal{F}\left(x, Q^{2}\right) \sim(\ln x)^{-3 / 2} x^{-4 \ln 2 \bar{\alpha}_{s}}$ as $x \rightarrow 0$, where $\alpha_{s}=\alpha_{s}\left(Q_{I}^{2}\right)$ and $Q^{2} \geq Q_{I}^{2}$. However, it was convincingly demonstrated in [20] (and discussed from a different point of view in [22]) that one only need keep a finite number of terms in the leading- $\ln (1 / x)$ series if working at finite $\ln (1 / x)$. In practice, if one works down to $x \approx 10^{-5}$ and the nonperturbative inputs behave roughly like $(1-x)^{5}$, then keeping terms up to $10_{\text {th }}$ order in the series for $\Gamma_{L L}^{0}\left(\alpha_{s} / N\right)$ and $\Gamma_{2 L}^{1}\left(\alpha_{s} / N\right)$ is more than sufficient. The series up to this order have the explicit form

$$
\Gamma_{L L}^{0}(x)=x+2.40 x^{4}+2.07 x^{6}+17.3 x^{7}+2.01 x^{8}+39.8 x^{9}+168.5 x^{10}+\cdots
$$


and

$2 \pi \Gamma_{2 L}^{1}(x)=1+2.5 x+x^{2}+x^{3}+7.01 x^{4}+5.81 x^{5}+13.4 x^{6}+58.1 x^{7}+64.7 x^{8}+196.8 x^{9}+650 x^{10}+\cdots$.

The low-order terms in both these series have coefficients which generally grow far less quickly than the asymptotic relationship $a_{n+1}=4 \ln 2 a_{n}$; some fall, and in the case of $\Gamma_{L L}^{0}\left(\alpha_{s} / N\right)$ are even zero. Thus, in the range of parameter space we are considering we will have rather less steep behaviour than the asymptotic limit of $\mathcal{F}\left(x, Q^{2}\right) \sim(\ln x)^{-3 / 2} x^{-4 \ln 2 \bar{\alpha}_{s}}$. Using the techniques in [22] one can derive analytic expressions for the structure functions in the small $-x$ limit. Choosing $Q_{I}^{2}=25 \mathrm{GeV}^{2}$ and making a guess that $A_{L L}=0.8 \mathrm{GeV}^{2}$ we obtain $\ln \left(Q_{I}^{2} / A_{L L}\right) \approx 3.4$. We also choose nonperturbative inputs which behave like $F_{i}(1-x)^{5}$ for $i=L, 2$. As argued in [22], for values of $x \lesssim 0.01$ it is a good approximation to replace the $(1-x)^{5}$ behaviour with the behaviour $\Theta(0.1-x) .{ }^{12}$ Doing this we can derive simple expressions for the form of the inputs for $x \lesssim 0.01$ which will be accurate up to a few percent error. Using the series (5.7) and (5.8) and the expressions (4.105) and (4.107) we can take the inverse Mellin transformations, obtaining

$$
\begin{aligned}
\mathcal{F}_{L}\left(x, Q_{I}^{2}\right) & \approx \alpha_{s}\left(Q_{I}^{2}\right) / 2 \pi\left[( F _ { L } + \frac { 4 } { 2 7 } F _ { 2 } ) \left(1+3.4 \bar{\alpha}_{s}\left(Q_{I}^{2}\right) \xi+5.8 \frac{\left(\bar{\alpha}_{s}\left(Q_{I}^{2}\right) \xi\right)^{2}}{2 !}+6.6 \frac{\left(\bar{\alpha}_{s}\left(Q_{I}^{2}\right) \xi\right)^{3}}{3 !}\right.\right. \\
& \left.\left.+13.7 \frac{\left(\bar{\alpha}_{s}\left(Q_{I}^{2}\right) \xi\right)^{4}}{4 !}+31.5 \frac{\left(\bar{\alpha}_{s}\left(Q_{I}^{2}\right) \xi\right)^{5}}{5 !}+56 \frac{\left(\bar{\alpha}_{s}\left(Q_{I}^{2}\right) \xi\right)^{6}}{6 !}+136 \frac{\left(\bar{\alpha}_{s}\left(Q_{I}^{2}\right) \xi\right)^{7}}{7 !}\right)-\frac{4}{27} F_{2}\right],
\end{aligned}
$$

and

$$
\begin{aligned}
\mathcal{F}_{2}\left(x, Q_{I}^{2}\right) & \approx 3.4 \alpha_{s}\left(Q_{I}^{2}\right) / 2 \pi\left(F_{L}+\frac{4}{27} F_{2}\right)\left(1+4.2 \bar{\alpha}_{s}\left(Q_{I}^{2}\right) \xi+7.1 \frac{\left(\bar{\alpha}_{s}\left(Q_{I}^{2}\right) \xi\right)^{2}}{2 !}+9.1 \frac{\left(\bar{\alpha}_{s}\left(Q_{I}^{2}\right) \xi\right)^{3}}{3 !}\right. \\
& \left.+19.7 \frac{\left(\bar{\alpha}_{s}\left(Q_{I}^{2}\right) \xi\right)^{4}}{4 !}+44.1 \frac{\left(\bar{\alpha}_{s}\left(Q_{I}^{2}\right) \xi\right)^{5}}{5 !}+84 \frac{\left(\bar{\alpha}_{s}\left(Q_{I}^{2}\right) \xi\right)^{6}}{6 !}+206 \frac{\left(\bar{\alpha}_{s}\left(Q_{I}^{2}\right) \xi\right)^{7}}{7 !}\right)+F_{2},
\end{aligned}
$$

where $\xi=\ln (0.1 / x)$, and $\alpha_{s}\left(Q_{I}^{2}\right) \approx 0.2$ if $\Lambda_{Q C D, 4} \approx 100 \mathrm{MeV}$. Putting in values of $F_{L}=2.5$ and $F_{2}=1$, choices which, as with the $(1-x)^{5}$ behaviour, are roughly compatible with the high $x$ data, we have a rough estimate of the form of the input structure functions at $Q_{I}^{2}=25 \mathrm{GeV}^{2}$. The coefficients in the series in $\alpha_{s}\left(Q_{I}^{2}\right) \xi$ multiplying $\left(F_{L}+4 / 27 F_{2}\right)$ grow a little more quickly for $\mathcal{F}_{2}\left(x, Q_{I}^{2}\right)$ than for $\mathcal{F}_{L}\left(x, Q_{I}^{2}\right)$. However, in the former case this contribution which rises with falling $x$ is accompanied by the flat $F_{2}$, whereas in the latter it is accompanied only by the nearly insignificant $-4 \alpha_{s}\left(Q_{I}^{2}\right) /(54 \pi) F_{2}$. Thus, for $x \approx 10^{-4} \mathcal{F}_{L}(x, 25)$ behaves approximately like $0.1 x^{-0.3}$ but $\mathcal{F}_{2}(x, 25)$ is slightly flatter, behaving approximately like $0.6 x^{-0.28}$. These powers of $x$ are clearly somewhat less steep than the asymptotic $x^{-0.5}$. Comparison of the estimate of $\mathcal{F}_{2}(x, 25)$ with the

12 The argument of the $\Theta$-function depends on the power of $(1-x)$. Higher powers would require the step to occur at lower $x$ and vice versa. 
data in [1] and [2] shows a very reasonable qualitative agreement. Of course, there is no data for $\mathcal{F}_{L}(x, 25)$ for values of $x$ anything like this low. Being rather more general, we find that for any $Q_{I}^{2}$ between $10 \mathrm{GeV}^{2}$ and $100 \mathrm{GeV}^{2}$, and with any sensible choice of $A_{L L}\left(\right.$ e.g $0.2 \mathrm{GeV}^{2} \geq A_{L L} \geq 2 \mathrm{GeV}^{2}$ ), then both $F_{2}\left(x, Q_{I}^{2}\right)$ and $F_{L}\left(x, Q_{I}^{2}\right)$ behave roughly like $x^{-0.3}$ for $0.01 \geq x \geq 0.00001$. Hence, this type of behaviour can be taken as a prediction of the theory.

One can also use the same techniques to make an estimate of the structure functions at values of $Q^{2}$ away from $Q_{I}^{2}$. With the particular inputs above they do lead to good qualitative agreement with the data below $x=0.01$, and we have every reason to feel encouraged by our results. However, the real test of our approach will be a complete global fit to the available data for $\mathcal{F}_{2}\left(x, Q^{2}\right)$ using the rather more accurate calculations, especially at large $x$, of the MRS program. We will therefore discuss these detailed fits next.

\section{Fits to the Data and Predictions.}

Once we have the general LORSC expression then by combining the singlet and nonsinglet components and varying all the free parameters $\left(A_{L L}\right.$, the soft inputs for $\mathcal{F}_{L}^{S}\left(x, Q^{2}\right)$ and $\mathcal{F}_{2}^{S}\left(x, Q^{2}\right)$ and the soft nonsinglet inputs), we obtain the best fit for the available $\mathcal{F}_{2}$ structure function data using a particular starting scale $Q_{I}^{2}$. Choosing the renormalization scale to be $Q^{2}$, the one-loop value for $\Lambda_{N_{f}=4}$ is fixed at $100 \mathrm{MeV}$, thus giving $\alpha_{s}\left(M_{Z}^{2}\right)=0.115 .{ }^{13}$ This precise value is not determined by a best fit, but a value near to this is certainly favoured.

There are some further details we should mention. Firstly, when obtaining a fit using the above approach, the values of $\mathcal{F}_{2}\left(x, Q^{2}\right)$ used are not precisely those published in [1] and [2]. This is because it is not $\mathcal{F}_{2}\left(x, Q^{2}\right)$ that is measured directly at HERA, but the differential cross-section. This is related to the structure functions as follows

$$
\frac{d^{2} \sigma}{d x d Q^{2}}=\frac{2 \pi \alpha^{2}}{Q^{4} x}\left(2-2 y+\frac{y^{2}}{1+R}\right) \mathcal{F}_{2}\left(x, Q^{2}\right),
$$

where $y=Q^{2} / x s \approx Q^{2} / 90000 x$ at HERA and $R=\mathcal{F}_{L}\left(x, Q^{2}\right) /\left(\mathcal{F}_{2}\left(x, Q^{2}\right)-\mathcal{F}_{L}\left(x, Q^{2}\right)\right)$. Over most of the range of parameter space $y$ is very small, $\mathcal{F}_{L}\left(x, Q^{2}\right)$ is likely to be small, and the measurement is essentially directly that of $\mathcal{F}_{2}\left(x, Q^{2}\right)$. However, at high $y$ the value of $\mathcal{F}_{2}\left(x, Q^{2}\right)$ must be extracted using some prescription for $\mathcal{F}_{L}\left(x, Q^{2}\right)$. Both H1 and ZEUS, roughly speaking, obtain their values of $\mathcal{F}_{L}\left(x, Q^{2}\right)$ from predictions coming from NLO calculations of $\mathcal{F}_{2}\left(x, Q^{2}\right)$. Since the approach in this paper leads in practice to a somewhat lower prediction of $\mathcal{F}_{L}\left(x, Q^{2}\right)$, the values of $\mathcal{F}_{2}\left(x, Q^{2}\right)$ used in the fit must be altered to take account of this. Thus, the $\mathcal{F}_{2}\left(x, Q^{2}\right)$ values are a little (at most about $6 \%$ ) lower for the largest values of $y$ than in those in [1] and [2].

13 Of course, since we are using a genuinely leading-order expression, any change in renormalization scale is exactly countered by a change in $\Lambda_{N_{f}=4}$. However, it is encouraging that making the simple choice $\mu_{R}^{2}=Q^{2}$ leads to a value of $\alpha_{s}\left(M_{Z}^{2}\right)$ which is nicely compatible with the usual value. 
The value of $m_{c}^{2}$ is chosen to be equal to $4 \mathrm{GeV}^{2}$ in order to obtain a reasonable description of the data on the charm structure function coming from EMC [42] and from measurements at HERA [43]. The quality of the fit is shown in fig. 1. It is of a fair quality, with the predicted $\mathcal{F}_{2}^{c}\left(x, Q^{2}\right)$ perhaps being a little large in general at large $x$, and a little small at small $x$, a result which is qualitatively consistent with the fact that we have used a threshold at $Q^{2}=m_{c}^{2}$, rather than at $W^{2}=Q^{2}\left(x^{-1}-1\right)=4 m_{c}^{2}$. Of course, $m_{c}^{2}=4 \mathrm{GeV}^{2}$ is a little high compared with the values obtained from reliable determinations. We will comment on this value later. The strange quark is treated as being massless in our calculations, which is presumably a good approximation for the values of $Q^{2}$ considered. Also, we insist that the strange contribution to the structure function is 0.2 of the singlet structure function minus the valence contributions (i.e. the "sea structure function") at $Q^{2}=m_{c}^{2}$. This ensures compatibility with the data on neutrino-induced deep inelastic di-muon production obtained by the CCFR collaboration [44].

Finally we consider the form of the gluon at large $x$. Within our effective factorization scheme we would expect the gluon distribution to be quite similar to that in the $\overline{\mathrm{MS}}$ scheme at NLO-in- $\alpha_{s}$ for very large $x$. Thus, we demand that our gluon is qualitatively similar to that obtained from the WA70 prompt photon data [45] at $x \geq 0.3$, i.e. roughly of the form $2.5(1-x)^{6}$ at $Q^{2}=20 \mathrm{GeV}^{2}$ for values of $x$ this large. Encouragingly, this is the type of large $x$ behaviour that the best fit chooses for the gluon, and no strong constraint is needed.

The fit is performed for a wide variety of data: the H1 [1] and Zeus [2] data on $\mathcal{F}_{2}^{e p}\left(x, Q^{2}\right)$ with $0.000032 \geq x \geq 0.32$ and $1.5 \mathrm{GeV}^{2} \geq Q^{2} \geq 5000 \mathrm{GeV}^{2}$; The BCDMS data [46] on $\mathcal{F}_{2}^{\mu p}\left(x, Q^{2}\right)$ with $0.07 \geq x \geq 0.75$ and $7.5 \mathrm{GeV}^{2} \geq Q^{2} \geq 230 \mathrm{GeV}^{2}$; the new NMC data [47] on $\mathcal{F}_{2}^{\mu p}\left(x, Q^{2}\right)$ and $\mathcal{F}_{2}^{\mu d}\left(x, Q^{2}\right)$ with $0.008 \geq x \geq 0.5$ and $2.5 \mathrm{GeV}^{2} \geq Q^{2} \geq 65 \mathrm{GeV}^{2}$; NMC data on the ratio of $\mathcal{F}_{2}^{\mu n}\left(x, Q^{2}\right)$ to $\mathcal{F}_{2}^{\mu p}\left(x, Q^{2}\right)$ [48] with $0.015 \geq x \geq 0.7$ and $5.5 \mathrm{GeV}^{2} \geq Q^{2} \geq 160 \mathrm{GeV}^{2}$, CCFR data [49] on $\mathcal{F}_{2}^{\nu N}\left(x, Q^{2}\right)$ and $\mathcal{F}_{3}^{\nu N}\left(x, Q^{2}\right)$ with $0.125 \geq x \geq 0.65$ and $5 \mathrm{GeV}^{2} \geq Q^{2} \geq 501.2 \mathrm{GeV}^{2}$; and the E665 [50] data on $\mathcal{F}_{2}^{\mu p}\left(x, Q^{2}\right)$ with $0.0037 \geq x \geq 0.387$ and $2.05 \mathrm{GeV}^{2} \geq 64.3 \mathrm{GeV}^{2}$. One can see that the full range of data used in the fit covers an extremely wide range of both $x$ and $Q^{2}$ and thus provides a very stringent test of any approach used to describe it. ${ }^{14}$

The result of the best fit to this data using the LORSC expressions with $Q_{I}^{2}$ chosen to be equal to $40 \mathrm{GeV}^{2}, m_{c}^{2}=4 \mathrm{GeV}^{2}$ and $m_{b}^{2}=20 \mathrm{GeV}^{2}$ is shown in table 1 . Also the result of the fit to the small $-x$ data is shown in fig. 2 . As one can see there is a very good quality fit to the whole selection of data, and thus over the whole range of $x$ and $Q^{2}$. Overall, the fit gives a $\chi^{2}$ of 1105 for 1099 data points. The fit shown is for the particular starting scale $Q_{I}^{2}=40 \mathrm{GeV}^{2}$, but the quality of the fit is extremely insensitive to changes in this scale, as we expect from the method of construction of the solutions. The fit is essentially unchanged over the range $20-80 \mathrm{GeV}^{2}$, and we choose $40 \mathrm{GeV}^{2}$ as

14 We have not included ZEUS data for $Q^{2} \geq 2000 \mathrm{GeV}^{2}$. 
the geometric mean. When $Q_{I}^{2}$ drops below $20 \mathrm{GeV}^{2}$ the fit immediately gets markedly worse, due to the bottom quark threshold.

There are 18 free parameters used in the fit: $\Lambda_{Q C D, 4}$ (which we choose to describe as a parameter since, although it is fixed at $100 \mathrm{MeV}$, and small variations would no doubt improve the fit slightly, it can certainly not vary too much); four parameters for each of the valence quark contributions, which are described by functions of the form (4.24) where the normalization is set by the number of valence quarks; four parameters for the nonperturbative inputs for the two singlet structure functions, which are of the form (5.6); and the unknown scale $A_{L L}$, where we allow $A_{L L}$ to be a free parameter for each $Q_{I}^{2}$. We do not consider $Q_{I}^{2}$ as a free parameter, since it can take a very wide range of values. The parameter $A_{L L}$, which we have argued should be a scale typical of soft physics, turns out to be $0.55 \mathrm{GeV}^{2}$ for the fit starting at $Q_{I}^{2}=40 \mathrm{GeV}^{2}$. This decreases a little as $Q_{I}^{2}$ increases and vice versa. For $Q_{I}^{2}=40 \mathrm{GeV}^{2}$ the soft inputs for the fit are roughly

$$
\hat{F}_{L}^{S}(x) \approx 3(1-x)^{5}\left(1+0.1 x^{0.5}-0.2 x\right), \quad F_{2}^{S}(x) \approx(1-x)^{3.4}\left(1-0.65 x^{0.5}+4.5 x\right) .
$$

These nonperturbative inputs lead to complete inputs of $\hat{F}_{L}^{S}\left(x, Q_{I}^{2}\right) \approx 3 x^{-0.33}$ and $F_{2}^{S}\left(x, Q_{I}^{2}\right) \approx$ $0.65 x^{-0.3}$ for $0.01 \geq x \geq 0.0001$, with the effective $\lambda$ increasing in both cases for even smaller $x$. Instead of forcing the nonperturbative inputs to be flat as $x \rightarrow 0$ we could allow an asymptotic behaviour $x^{-\lambda}$, where $\lambda \lesssim 0.08$. This leads to an equally good fit.

Thus, the LORSC fit seems to be a success. However, in order to gauge its true quality it is helpful to have some points of comparison, and we will discuss some alternative fits. The first we mention is that obtained by simply solving the evolution equations for structure functions using the full leading order physical anomalous dimensions (4.91), rather than using the more careful procedure described above. In this case if we drop the restriction on the relationship between the inputs and let them each have arbitrary small $x$ form (the arguments relating the inputs do not really follow if we simply solve using the full physical anomalous dimensions) then the fit is nearly as good as the LORSC fit for $Q^{2} \geq 4 \mathrm{GeV}^{2}$. However, it gets markedly worse for $Q^{2} \sim 3 \mathrm{GeV}^{2}$ and is extremely poor below this. If we do try to impose the relationship between the inputs (i.e. retain any explanatory power for the form of these inputs), then the fit at small $x$ is much worse than the LORSC fit, the value of $\left(d \mathcal{F}_{2}\left(x, Q^{2}\right) / d \ln Q^{2}\right)$ being rather too steep. Thus, phenomenologically the naive use of the LO physical anomalous dimensions is clearly inferior to the full LORSC calculation.

We also compare to more conventional approaches. As in [30], the most recent MRS fits $\mathrm{R}_{1}$ and $\mathrm{R}_{2}$ are shown. These are obtained using the standard two-loop method, where $\mathrm{R}_{1}$ allows $\Lambda_{\overline{\mathrm{MS}}}^{N_{f}=4}$ to be free (giving $\Lambda_{\overline{\mathrm{MS}}}^{N_{f}=4}=241 \mathrm{MeV}$ ) and $\mathrm{R}_{2}$ fixes $\Lambda_{\overline{\mathrm{MS}}}^{N_{f}=4}=344 \mathrm{MeV}$ to force a better fit to the HERA data. The MRS fits are useful for purposes of comparison for a number of reasons: the treatment of the errors in this paper is identical to that in the MRS fits, so consistency in this respect is guaranteed, any systematic differences due to differences in computer programs is 
guaranteed to be absent, and the cuts in $Q^{2}$ for each data set are chosen to be the same in this paper as for the MRS fits. The number of free parameters in the NLO-in- $\alpha_{s}$ fit is the same as in the LORSC fit. There is one more parameter in the NLO-in- $\alpha_{s}$ fit due to the powerlike forms of the input gluon and singlet quark at small $x$ being independent ${ }^{15}$ whereas the small- $x$ shape of the inputs for both structure functions is completely determined by the one parameter $A_{L L}$ in the LORSC fit. However, there is one less parameter in the NLO fit due to the normalization of the gluon being determined by momentum conservation. Essentially, the LORSC fit is a little less constrained at large $x$ than the NLO fit, but rather more predetermined at small $x$.

Comparing the LORSC fit and the MRS fits, it is clear that the LORSC scheme-independent fit is much better for the HERA data (even when compared to $\mathrm{R}_{2}$ ), much better for the BCDMS data (even when compared to $\mathrm{R}_{1}$ ) and similar in standard for the rest of the data. The overall fit is $\sim 200$ better for the whole data set; clearly a lot better. However, this direct comparison with the MRS fits is rather unfair: the MRS fits use the SLAC data [51] on $\mathcal{F}_{2}^{e p}\left(x, Q^{2}\right)$ which is not included in the LORSC fit due to much greater sensitivity of this data to potential higher twist effects than any of the other data sets, and also the fact that the quark and gluon distributions are input at $1 \mathrm{GeV}^{2}$ in the MRS fits is not helpful to their quality. If the best fit is obtained for the HERA data using the NLO calculation with massless quarks with inputs at $Q_{I}^{2} \approx 4 \mathrm{GeV}^{2}$, as in [27], and evolution performed downwards in $Q^{2}$ then the gluon becomes negative at very small $x$ before $Q^{2}=1 \mathrm{GeV}^{2}$. Hence, starting at larger $Q^{2}$ where the gluon distribution is happy to be positive everywhere, produces a better fit. Thus, in order to make a more meaningful comparison of the LORSC fit with a NLO-in- $\alpha_{s}$ fit we have ourselves performed a NLO-in- $\alpha_{s}$ fit, called $\mathrm{NLO}_{1}$, allowing the normalizations to vary in the same way as in the LORSC fit, with exactly the same treatment of quark thresholds as the LORSC fit, with the input parton distributions chosen at $Q_{I}^{2}=m_{c}^{2}$ and with the fit to exactly the same data. ${ }^{16}$ As in the MRS fits the values of $\mathcal{F}_{2}\left(x, Q^{2}\right)$ at small $x$ are those quoted in [2] and [1]. The value of $m_{c}^{2}$ is chosen to provide a good description of the data on the charm structure function. The value needed is $m_{c}^{2}=2.75 \mathrm{GeV}^{2}$, and the fit to the charm structure function data is shown in fig. 3. ${ }^{17}$ The value of $\Lambda_{\overline{\mathrm{MS}}}^{N_{f}}=4$ determined by the fit is $299 \mathrm{MeV}$. The quality of the fit is shown in table 1 .

15 We claim that the allowed powerlike form of the inputs at small $x$ is against the spirit of a well-ordered perturbative expansion, as already discussed in $\S 4.1$. However, enforcing this rule would mean that the quality of the fit to the small- $x$ data was very poor. Thus, we allow the more usual, unjustified choice for the small- $x$ form of the inputs. In fact, at $Q_{I}^{2}=1 \mathrm{GeV}^{2}$ the gluon is strongly valence-like, while the sea-quark distribution $\sim x^{-1-0.15}$ at small $x$.

16 Once again we allow the small- $x$ form of the inputs to be unjustified powerlike behaviours. At $Q_{I}^{2}=2.75 \mathrm{GeV}^{2}$ the gluon is quite flat while the sea-quark distribution $\sim x^{-1-0.22}$.

17 As in the LORSC fit the choice of $m_{c}^{2}$ which gives a good description of the charm data is also the choice which gives the best global fit. 
The higher starting scale for evolution has produced a better fit to the HERA data than the MRS fits. However, the best fit comes from allowing the normalization of the H1 data to be at the lower limit allowed by the error on the normalization ${ }^{18}$, and is still not as good as the LORSC fit to the HERA data. Not including the SLAC data leads to a much better fit to the BCDMS data than the MRS fits, but again this is clearly not as good as the LORSC fit. The fit to the NMC data is much the same for the LORSC fit as for the $\mathrm{NLO}_{1}$ fit, and the $\mathrm{NLO}_{1}$ fit is a little better for the CCFR data. The overall quality of the $\mathrm{NLO}_{1}$ fit is 1169 for the 1099 points, and thus is 64 worse than the LORSC fit. Therefore, the LORSC fit is still clearly better than the $\mathrm{NLO}_{1}$ fit, but not nearly as convincingly as appeared to be the case when compared to the MRS fits. Nevertheless, it is encouraging that, while the overall fits to the relatively high $x$ data, i.e. the BCDMS, NMC, E665 and CCFR data are similar in the $\mathrm{NLO}_{1}$ fit and the LORSC fit, it is the fit to the small- $x$ HERA data that is definitely better for the LORSC fit, as we would expect. This can be seen even more clearly if we examine the quality of the fit by separating the data into two sets: one where $x<0.1$ and one where $x \geq 0.1$. This is shown in table 2 , and demonstrates that the LORSC fit is superior at small $x$, while not quite as good as the $\mathrm{NLO}_{1}$ fit at large $x$.

This qualitative result is exactly what we would expect. The importance of the leading $-\ln (1 / x)$ terms in the LORSC calculation can be quantitatively judged by how they affect the fit. If, after obtaining the best LORSC fit, all terms other than those in the one-loop expressions are set to zero, the quality of the fit is unchanged above $x=0.3$, begins to alter slightly below this, and is clearly worse by the time we reach $x=0.1$. Thus, the leading- $\ln (1 / x)$ terms are important by this value of $x$. However, most of this effect is due to the terms at $\mathcal{O}\left(\alpha_{s}^{2}\right)$, so the NLO expression at fixed order in $\alpha_{s}$ should be insensitive to higher-order-in- $\alpha_{s}$ leading- $\ln (1 / x)$ terms down to $x$ somewhat lower than 0.1. Therefore, above $x=0.1$ the NLO fit should in principle be better than the LORSC fit since it contains terms at NLO in $\alpha_{s}$ which are important at large $x$. However, the NLO fit should be considerably worse at small $x$ since it does not contain many important leading $-\ln (1 / x)$ terms. This is qualitatively in agreement with the comparison of the $\mathrm{NLO}_{1}$ fit and the LORSC fit.

However, the above comparison is somewhat incorrect because in the process of obtaining the best fit for all the data the $\mathrm{NLO}_{1}$ fit may choose some parameters, particularly $\Lambda_{\overline{\mathrm{MS}}}^{N_{f}}=4$, such that they mimic the effects of the leading $-\ln (1 / x)$ terms, and a decent fit for the small $x$ data is obtained to the detriment of the fit to the large $x$ data. In order to check this hypothesis we have also performed a NLO-in- $\alpha_{s}$ fit with $\Lambda_{\overline{\mathrm{MS}}}^{N_{f}=4}$ fixed at $250 \mathrm{MeV}$, which we will denote by $\mathrm{NLO}_{2}$. The results of this fit in terms of the different data sets is shown in table 1 . The fit clearly improves compared to the $\mathrm{NLO}_{1}$ fit for the BCDMS and CCFR data, and gives the best overall fit for the high $x$ data sets. It worsens somewhat for the NMC data, and also for the HERA data, and overall

18 The fit to the H1 data continues to improve slightly for a normalization going down to 0.96 . 
is slightly worse than the $\mathrm{NLO}_{1}$ fit, having a $\chi^{2}$ of 1184 for the 1099 data points. Nevertheless, it is perhaps a truer representation of a real NLO-in- $\alpha_{s}$ fit than $\mathrm{NLO}_{1}$ since it gives a better fit to high $x$ data, but not such a good fit to the small- $x$ data which presumably require the leading- $\ln (1 / x)$ terms. Whether one believes this argument or not, it is certainly clear that the LORSC fit does provide a better description of the data than any standard NLO-in- $\alpha_{s}$ fit.

This leads us to the question of the determination of $\alpha_{s}\left(M_{Z}^{2}\right)$ using global fits to structure function data. The complete RSC expression for structure functions only exists at leading order, and this leaves the renormalization scale undetermined. Hence, a determination of $\alpha_{s}\left(M_{Z}^{2}\right)$ does not really take place. It is not yet possible to extend the RSC calculation beyond the leading order due to lack of knowledge of NLO-in- $\ln (1 / x)$ terms, but hopefully these will shortly become available [36], and when they do the NLO versions of (4.105)-(4.107) can be derived and put to use, and the NLO coupling constant determined. Until these full renormalization-scheme-consistent NLO expressions become available, we believe that it is incorrect to use the present NLO-in- $\alpha_{s}$ fits to small $-x$ structure function data in order to determine the NLO coupling constant. However, as already argued, the fixed-order-in- $\alpha_{s}$ expressions should be accurate for CCFR, BCDMS and NMC data (except perhaps at the lowest $x$ values), which after all are still much more precise than HERA data, and fits to these data alone will provide the best determination of the NLO $\alpha_{s}\left(Q^{2}\right)$.

We have given what we hope are convincing arguments for our advocated approach for calculating structure functions. Not only do we claim theoretical correctness, and limited predictive power, but we also have good quality, very comprehensive fits to data on $\mathcal{F}_{2}\left(x, Q^{2}\right)$. However, we are well aware that only further experimental tests can prove us right (or wrong). Hence, we now discuss our predictions for $\mathcal{F}_{L}\left(x, Q^{2}\right)$.

So far we have only probed $\mathcal{F}_{L}\left(x, Q^{2}\right)$ indirectly, i.e. it is simply related to the $\ln Q^{2}$ derivative of $\mathcal{F}_{2}\left(x, Q^{2}\right)$ (as well as to the input $\mathcal{F}_{2}\left(x, Q_{I}^{2}\right)$ ). Having tied down the nonperturbative inputs and $A_{L L}$ and $Q_{I}^{2}$ from our fit to $\mathcal{F}_{2}\left(x, Q^{2}\right)$, we have a prediction for $\mathcal{F}_{L}\left(x, Q^{2}\right)$. The result of this prediction for the fit with $Q_{I}^{2}=40 \mathrm{GeV}^{2}$ is shown in fig. 4, where it is compared to the prediction using the NLO-in $-\alpha_{s}$ approach, in particular the $\mathrm{NLO}_{1}$ fit. As one can see, it is smaller than the $\mathrm{NLO}_{1} \mathcal{F}_{L}\left(x, Q^{2}\right)$, but becomes steeper at very small $x$. The $\mathrm{NLO}_{2}$ fit gives a very similar form of $\mathcal{F}_{L}\left(x, Q^{2}\right)$ to the $\mathrm{NLO}_{1}$ fit. The LORSC prediction for $\mathcal{F}_{L}\left(x, Q^{2}\right)$ is weakly dependent on the value of $Q_{I}^{2}$ chosen: the value at $Q^{2}=5 \mathrm{GeV}^{2}$ and $x=10^{-4}$ varies by $\pm 10 \%$ within our range of $Q_{I}^{2}$ (increasing with $Q_{I}^{2}$ ), and by less than this for higher $x$ and $Q^{2}$.

Hence, measurements of $\mathcal{F}_{L}\left(x, Q^{2}\right)$ at $x<10^{-2}$ would be a good discriminant between fixedorder-in- $\alpha_{s}$ calculations and those involving leading $-\ln (1 / x)$ terms. However, the "determination" of $\mathcal{F}_{L}\left(x, Q^{2}\right)$ already performed by $\mathrm{H} 1$ [52] is really only a consistency check for a particular NLOin- $\alpha_{s}$ fit, and is by no means a true measurement of $\mathcal{F}_{L}\left(x, Q^{2}\right)$. In essence, all it proves is that the measurements of the cross-section are consistent with a particular NLO-in- $\alpha_{s}$ fit to $\mathcal{F}_{2}\left(x, Q^{2}\right)$ when the relationship between the cross-section and the value of $\mathcal{F}_{2}\left(x, Q^{2}\right)$ is determined assuming the 
correctness of the NLO-in- $\alpha_{s}$ expressions for both structure functions. This is a perfectly correct procedure, and should be adopted for any fit to $\mathcal{F}_{2}\left(x, Q^{2}\right)$ data, as it has been for the LORSC fit, but in itself says nothing about the validity of a different approach, or about the actual value of $\mathcal{F}_{L}\left(x, Q^{2}\right)$. Hence, real measurements of $\mathcal{F}_{L}\left(x, Q^{2}\right)$ are needed in order to find the real values of $\mathcal{F}_{L}\left(x, Q^{2}\right)$. From fig. 4 it is clear that such measurements at HERA would be an important (and probably essential) way of determining the validity of the approach in this paper, and the genuine importance of leading $-\ln (1 / x)$ terms in structure functions.

Another potentially important discriminant between different methods of calculating structure functions is the measurement of the charm structure function. Both the LORSC fit and the $\mathrm{NLO}_{1}$ fit can provide good fits to the currently available charm data, as already seen, and the value of $m_{c}^{2}$ in the $\mathrm{NLO}_{1}$ fit is rather more satisfactory than that in the LORSC fit. However, these calculations involve incorrect treatments of the charm quark threshold. A more correct treatment of this threshold at NLO in $\alpha_{s}$ [53] shows that the value of $m_{c}^{2}$ must be considerably reduced in order to produce the same sort of value of the charm structure function as the approach used in this paper. Indeed, as seen in [54], NLO-in- $\alpha_{s}$ calculations with $m_{c}^{2}=2.25 \mathrm{GeV}^{2}$ undershoot the small- $x$ data. A correct treatment of the charm quark threshold within the framework advocated in this paper has not been completely worked out (although, as already mentioned, work is in progress), and has certainly not been tested. However, a comparison of the theoretical calculations with the ever-improving data on the charm structure function seems potentially to be a very useful way of discriminating between different methods of calculation.

In principle there are many other quantities which could be calculated within the LORSC framework and compared to those calculated using the NLO-in- $\alpha_{s}$ approach (or any other method)

and to experimental data. Particularly obvious examples are the distribution of the transverse energy flow in the final state in lepton-hadron scattering and the the cross-section for forward jet production, for both of which there exists some experimental data which does not seem to be terribly well described by the order-by-order-in- $\alpha_{s}$ approach, e.g. [55] and [56]. We have not performed a LORSC calculation of any such quantities, and it is very difficult to estimate the results, other than guess that they will probably lie somewhere between the fixed-order-in- $\alpha_{s}$ predictions and those obtained using BFKL physics naively. Such calculations are obviously a priority, and work will begin soon. Only by comparing our theoretical predictions with a wide variety of experimental data can we determine unambiguously which theoretical approach is correct.

\section{Conclusion and Summary.}

In this paper we have derived expressions for the structure functions $\mathcal{F}_{2}\left(x, Q^{2}\right)$ and $\mathcal{F}_{L}\left(x, Q^{2}\right)$ in a theoretically correct, well-ordered manner, within the framework of the renormalization group and collinear factorization. We have first done this for the particular expansions schemes which 
order the expressions strictly in orders of $\alpha_{s}$, i.e. the standard loop expansion, or in terms of the leading powers of $\ln (1 / x)$ for given power of $\alpha_{s}$, i.e. the leading- $\ln (1 / x)$ expansion. In both cases we have demonstrated that a correct calculation in terms of structure function inputs and evolution automatically leads to factorization-scheme-invariant results which may be expressed in terms of physical anomalous dimensions. Thus, these physical anomalous dimensions are fundamental pieces in the correct expressions for the structure functions. However, we also demonstrate that in the case of the leading $-\ln (1 / x)$ expansion the correct expressions are more difficult to obtain than in the loop expansion, and in both cases a correct calculation requires rather more care than just the use of these physical anomalous dimensions.

We have then argued that both the above expansion schemes are restrictive, and lead to only part of the correct solution at any given order. We have shown that the only calculational method which is truly consistent with working to a given order in $\alpha_{s}$ within a given renormalization scheme is the renormalization-scheme-consistent expansion, in which one works to a given order in both $\alpha_{s}$, and in $\ln (1 / x)$ for given power of $\alpha_{s}$, for physical quantities, i.e. the inputs and evolution of the structure functions. Doing this one obtains the conventional, order-by-orderin $-\alpha_{s}$ renormalization group results in the high $x$ limit, and smoothly incorporates the leading $\ln (1 / x)$ corrections. We have then derived the leading-order, renormalization-scheme-consistent (and hence renormalization scheme invariant) expressions for the structure functions $\mathcal{F}_{2}\left(x, Q^{2}\right)$ and $\mathcal{F}_{L}\left(x, Q^{2}\right)$. In this derivation we have made use of the physical anomalous dimensions (which is not necessary, but is extremely convenient), but needed to do more work than simply include them in their leading form and solve the evolution equations. Indeed, this naive method of inclusion leads to a comparison with data which is far worse than that obtained from the correct LORSC calculation.

As part of our overall approach we have also taken an unusual view of the starting scale $Q_{I}^{2}$. Rather than trying to guess some form for the input at some particular $Q_{I}^{2}$, or simply allowing the inputs at some arbitrary $Q_{I}^{2}$ to take any form they wish within a given parameterization, we have demanded firstly that our expressions should be formally insensitive to the choice of the input scale, and secondly that any deviation from the flat Regge-type behaviour of the structure functions must come from perturbative effects. Thus, our inputs take the form of nonperturbative functions, which are flat at small $x$, convoluted with functions of the physical anomalous dimensions which are evaluated at $Q_{I}^{2}$, and determined by the requirement of insensitivity to the value of $Q_{I}^{2}$. This leads to our inputs being determined entirely in terms of the flat nonperturbative inputs and one arbitrary scale $A_{L L}$ which roughly indicates the scale where perturbative physics should break down, i.e. $A_{L L} \lesssim 1 \mathrm{GeV}^{2}$. This gives us a great deal more predictive power than more usual approaches. We have some idea of the form of individual structure functions at small $x$ : for a range of sensible choices of $Q_{I}^{2}\left(10 \mathrm{GeV}^{2}-100 \mathrm{GeV}^{2}\right)$ and $A_{L L}$ we obtain $\mathcal{F}_{2}\left(x, Q_{I}^{2}\right)$ roughly $\propto x^{-(0.25-0.33)}$ for $0.01 \geq x \geq 0.00001$, which is clearly in good qualitative agreement with the data. However, we 
also have a much stronger prediction for the relationship between the small- $x$ inputs. So as well as some predictive (or at the very least, explanatory) power for individual structure functions, once we choose the input for one, in practice $\mathcal{F}_{2}\left(x, Q^{2}\right)$, we have determined, up to a small amount of freedom, the small- $x$ inputs for $d \mathcal{F}_{2}\left(x, Q^{2}\right) / d \ln Q^{2}$ and $\mathcal{F}_{L}\left(x, Q^{2}\right)$.

Not only are the features of the LORSC calculation compelling, but they also work rather well in practice (for a range of $Q_{I}^{2}$ from $20 \mathrm{GeV}^{2}-80 \mathrm{GeV}^{2}$ ). The LORSC expressions, including this constraint on the small- $x$ inputs, lead to very good fits to the data, the $\chi^{2}$ for the LORSC fit to 1099 data on $\mathcal{F}_{2}\left(x, Q^{2}\right)$ ranging from from $0.75 \geq x \geq 0.000032$ and $1.5 \mathrm{GeV}^{2} \leq Q^{2} \leq 5000 \mathrm{GeV}^{2}$ is better by at least 60 than any NLO-in- $\alpha_{s}$ fit, a very significant improvement. This is even though the NLO-in $-\alpha_{s}$ fit is allowed arbitrary, unjustified powerlike behaviour at small $x$, and the small- $x$ inputs for $\mathcal{F}_{2}\left(x, Q^{2}\right)$ and $d \mathcal{F}_{2}\left(x, Q^{2}\right) / d \ln Q^{2}$ are largely independent. In fact, all of this superiority comes from the fit to the data with $x<0.1$. Thus, we find the naively expected, but much refuted, result that the leading $\ln (1 / x)$ terms are both important and helpful in fits to structure functions when included properly. As a caveat, it is certainly true that the calculations in this paper must be improved to take account of massive quark thresholds in a better manner (as is also the case with NLO-in- $\alpha_{s}$ fits), and work towards this end is in progress. Nevertheless, with the present treatment we feel that the quality of the fit and the degree of explanatory (if not predictive) power, not to mention the theoretical correctness, give strong justification for the LORSC expressions.

We do, however, recognize that the quality of the fit alone does not necessarily convince one that this approach has to be correct. In order to obtain verification we must exploit the factorization theory fully and compare with more and different experimental data. Hence, we have presented a LORSC prediction for $\mathcal{F}_{L}\left(x, Q^{2}\right)$, comparing it to that obtained using the NLO-in- $\alpha_{s}$ approach. Hopefully there will be true measurements of $\mathcal{F}_{L}\left(x, Q^{2}\right)$ at HERA some time in the future with which we can compare these predictions. We stress the importance of such measurements to the understanding of the physics which really underlies hadron interactions. In the near future we will also have predictions of the charm contribution to the structure function within the framework of a correct treatment of the massive quark, and comparison with the ever-improving data on the charm structure function should also be a good discriminant between different theoretical approaches. Calculations and measurements of other, less inclusive quantities, such as forward jets, are another clear goal.

Finally, our calculation is at present only at leading order due to the lack of knowledge of next-to-leading-order-in- $\ln (1 / x)$ coefficient functions and anomalous dimensions, or equivalently of physical anomalous dimensions. This means that the NLO-in- $\alpha_{s}$ approach is still in principle superior to our approach at high $x$, where the leading- $\ln (1 / x)$ terms at third order in $\alpha_{s}$ and beyond are not important but the $\mathcal{O}\left(\alpha_{s}^{2}\right)$ terms are. Indeed, in practice the NLO-in- $\alpha_{s}$ fits, are slightly better than the LORSC fit for data at $x \geq 0.1$, and we might expect any predictions coming from the NLO-in- $\alpha_{s}$ approach to be more accurate than those coming from the LORSC approach down 
to values of $x$ somewhere in the region of 0.05. The lack of the NLORSC expressions also means that a true determination of $\alpha_{s}\left(M_{Z}^{2}\right)$ from a global fit to structure function data is not yet possible, but that the best determination from fits to structure function data should at present come from using the NLO-in- $\alpha_{s}$ approach, using only large $x$ data. For a really fair comparison between the renormalization-scheme-consistent method and the conventional order-by-order-in- $\alpha_{s}$ approach we really need the full NLORSC calculation. Hopefully the required NLO in $\ln (1 / x)$ quantities will soon become available, and with some work we will be able to make such a comparison. We would expect that when using the NLORSC expressions the fit to the large $x$ data would become at least as good as for the NLO-in- $\alpha_{s}$ approach, and that the fit to the small- $x$ data would be of at least the same quality as for the LORSC fit. We wait expectantly to discover if this is indeed the case.

\section{Acknowledgements.}

I would like to thank R.G. Roberts for continual help during the period of this work and for the use of the MRS fit program. I would also like to thank Mandy Cooper-Sarkar, Robin Devenish, Jeff Forshaw and Werner Vogelsang for helpful discussions. 


\section{Table 1}

Comparison of quality of fits using the full leading-order (including leading- $\ln (1 / x)$ terms) renormalization-scheme-consistent expression, LORSC, and the two-loop fits $\mathrm{MRSR}_{1}, \mathrm{MRSR}_{2}$, $\mathrm{NLO}_{1}$ and $\mathrm{NLO}_{2}$. For the LORSC fit the $\mathrm{H} 1$ data chooses a normalization of 1.00, the ZEUS data of 1.015, and the BCDMS data of 0.975. The CCFR data is fixed at a normalization of 0.95, and the rest is fixed at 1.00. Similarly, for the $\mathrm{NLO}_{1}$ fit the $\mathrm{H} 1$ data is fixed at a normalization of 0.985 , the ZEUS chooses a normalization of 0.99, and the BCDMS data of 0.975. Again the CCFR data is fixed at a normalization of 0.95 , and the rest fixed at 1.00. Also, for the $\mathrm{NLO}_{2}$ fit the $\mathrm{H} 1$ data is fixed at a normalization of 0.985 , the ZEUS chooses a normalization of 0.985 , and the BCDMS data of 0.97. Again the CCFR data is fixed at a normalization of 0.95, and the rest fixed at 1.00. In the $R_{1}$ and $R_{2}$ fits the BCDMS data has a fixed normalization of 0.98 , the CCFR data of 0.95 and the rest of 1.00 .

\begin{tabular}{|l|r|r|r|r|r|r|}
\hline Experiment & $\begin{array}{r}\text { data } \\
\text { points }\end{array}$ & \multicolumn{5}{|c|}{$\chi^{2}$} \\
& LORSC & $\mathrm{NLO}_{1}$ & $\mathrm{NLO}_{2}$ & $\mathrm{R}_{1}$ & $\mathrm{R}_{2}$ \\
\hline $\mathrm{H} 1 \mathcal{F}_{2}^{e p}$ & 193 & 123 & 145 & 145 & 158 & 149 \\
ZEUS $\mathcal{F}_{2}^{e p}$ & 204 & 253 & 281 & 296 & 326 & 308 \\
\hline $\mathrm{BCDMS} \mathcal{F}_{2}^{\mu p}$ & 174 & 181 & 218 & 192 & 265 & 320 \\
$\mathrm{NMC} \mathcal{F}_{2}^{\mu p}$ & 129 & 122 & 131 & 148 & 163 & 135 \\
$\mathrm{NMC} \mathcal{F}_{2}^{\mu d}$ & 129 & 114 & 107 & 125 & 134 & 99 \\
$\mathrm{NMC} \mathcal{F}_{2}^{\mu n} / \mathcal{F}_{2}^{\mu p}$ & 85 & 142 & 137 & 138 & 136 & 132 \\
$\mathrm{E} 665 \mathcal{F}_{2}^{\mu p}$ & 53 & 63 & 63 & 63 & 62 & 63 \\
\hline $\mathrm{CCFR} \mathcal{F}_{2}^{\nu N}$ & 66 & 59 & 48 & 40 & 41 & 56 \\
$\mathrm{CCFR} \mathcal{F}_{2}^{\nu N}$ & 66 & 48 & 39 & 36 & 51 & 47 \\
\hline
\end{tabular}

\section{Table 2}

Comparison of quality of fits using the full leading-order (including leading- $\ln (1 / x)$ terms) renormalization-scheme-consistent expression, LORSC, and the two-loop fits $\mathrm{NLO}_{1}$ and $\mathrm{NLO}_{2}$. The fits are identical to above, but the data are presented in terms of whether $x$ is less than 0.1 or not.

\begin{tabular}{|l|r|r|r|r|}
\hline & $\begin{array}{r}\text { data } \\
\text { points }\end{array}$ & \multicolumn{3}{|c|}{$\chi^{2}$} \\
& LORSC & $\mathrm{NLO}_{1}$ & $\mathrm{NLO}_{2}$ \\
\hline$x \geq 0.1$ & 551 & 622 & 615 & 595 \\
$x<0.1$ & 548 & 483 & 554 & 589 \\
\hline total & 1099 & 1105 & 1169 & 1184 \\
\hline
\end{tabular}




\section{References}

[1] H1 collaboration, Nucl. Phys. B470 (1996) 3.

[2] ZEUS collaboration: M. Derrick et al, Zeit. Phys. C69 (1996) 607;

preprint DESY 96-076 (1996), Zeit. Phys. C, in print.

[3] G. Altarelli and G. Parisi, Nucl. Phys. B126 (1977) 298;

Yu.L. Dokshitzer, Sov. Jour. JETP 46 (1977) 641;

L.N.Lipatov, Sov. Jour. Nucl. Phys. 20 (1975) 95;

V.N. Gribov and L.N. Lipatov, Sov. Jour. Nucl. Phys. 15 (1972) 438.

[4] A. Donnachie and P.V. Landshoff, Nucl. Phys. B244 (1984) 322; Nucl. Phys. B267 (1986) 690.

[5] A.D. Martin, R.G. Roberts and W.J. Stirling, Phys. Rev. D47 (1993) 867; Phys. Lett. B306 (1993) 145.

[6] A. de Rújula et al, Phys. Rev. D10 (1974) 1649.

[7] L.N. Lipatov, Sov. Jour. Nucl. Phys. 23 (1976) 338;

E.A. Kuraev, L.N. Lipatov and V.S. Fadin Sov. Jour. JETP 45 (1977) 199;

Ya. Balitskii and L.N. Lipatov, Sov. Jour. Nucl. Phys. 28 (1978) 6.

[8] A.D Martin, R.G. Roberts and W.J. Stirling, Phys. Lett. B354 (1995) 155;

H.L. Lai et al, Phys. Rev. D51 (1995) 4763.

[9] M. Glück, E. Reya and A. Vogt, Zeit. Phys. C48 (1990) 471; Zeit. Phys. C53 (1992) 127;

Phys. Lett. B306 (1993) 391; Zeit. Phys. C67 (1995) 433.

[10] R.D. Ball and S. Forte, Phys. Lett. B335 (1994) 77.

[11] R.D. Ball and S. Forte, Phys. Lett. B336 (1994) 77.

[12] J.C. Collins, D.E. Soper and G. Sterman, in: Perturbative Quantum Chromodynamics, ed. A.H. Mueller (World Scientific, Singapore, 1989), and references therein.

[13] S. Catani, M. Ciafaloni and F. Hautmann, Phys. Lett. B242 (1990) 97; Nucl. Phys. B366 (1991) 135; Phys. Lett. B307 (1993) 147.

[14] J.C. Collins and R.K. Ellis, Nucl. Phys. B360 (1991) 3.

[15] A.J. Askew, J. Kwieciński, A.D. Martin and P.J. Sutton, Phys. Rev. D49 (1994) 4402;

A.J. Askew et al, Phys. Lett. B325 (1994) 212.

[16] J. Kwieciński, A.D. Martin and P.J. Sutton, Phys. Rev. D53 (6094) 1996; Zeit. Phys. C71 (1996) 585.

[17] M. Ciafaloni, Nucl. Phys. B296 (1988) 49;

S. Catani, F. Fiorani and G. Marchesini, Phys. Lett. B234 (1990) 339; Nucl. Phys. B336 (1990) 18.

[18] S. Catani and F. Hautmann, Phys. Lett. B315 (1993) 157; Nucl. Phys. B427 (1994) 475.

[19] T. Jaroszewicz, Phys. Lett. B116 (1982) 291.

[20] R.D. Ball and S. Forte, Phys. Lett. B351 (1995) 313; Phys. Lett. B358 (1995) 365.

[21] R.K. Ellis, F. Hautmann and B.R. Webber, Phys. Lett. B348 (1995) 582.

[22] J.R. Forshaw, R.G. Roberts and R.S. Thorne, Phys. Lett. B356 (1995) 79.

[23] J. Blümlein, S. Riemersma and A. Vogt, Proc. of the International Workshop QCD and QED in higher orders, Rheinsburg, Germany, April, 1996, eds. J. Blümlein, F. Jegerlehner and T. 
Riemann, Nucl. Phys. B (Proc. Suppl.), 51C (1996) p. 30; hep-ph/9607329, Proc. of the International Workshop on Deep Inelastic Scattering, Rome, April, 1996, in print.

[24] S. Catani, Zeit. Phys. C70 (1996) 263.

[25] M. Ciafaloni, Phys. Lett. B356 (1995) 74.

[26] A.D. Martin, W.J. Stirling and R.G. Roberts, Phys. Lett. B387 (1996) 419.

[27] R.D. Ball and S. Forte, hep-ph/9607289, Proc. of the International Workshop on Deep Inelastic Scattering, Rome, April, 1996, in print.

[28] S. Forte and R.D. Ball, hep-ph/9607291, Proc. of the International Workshop on Deep Inelastic Scattering, Rome, April, 1996, in print.

[29] I. Bojak and M. Ernst, hep-ph/9609378, preprint DO-TH 96/18, September 1996.

[30] R.S. Thorne, Phys. Lett. B3921997463.

[31] S. Catani, talk at UK workshop on HERA physics, September 1995, unpublished; hepph/9609263, preprint DDF 248/4/96, April 1996; hep-ph/9608310, Proc. of the International Workshop on Deep Inelastic Scattering, Rome, April, 1996, in print.

[32] R.S. Thorne, RAL-TR-96-065, hep-ph/9701241..

[33] A.D. Martin, R.G. Roberts and W.J. Stirling, Phys. Lett. B266 (1991) 173.

[34] G. Altarelli, R.K. Ellis and G. Martinelli, Nucl. Phys. B157 (1979) 461.

[35] J. Blümlein et al, Proc. of the 1996 HERA Physics Workshop, eds. G. Ingelman, R. Klanner, and A. De Roeck, DESY, Hamburg, 1996, Vol. 1, p. 23.

[36] L.N. Lipatov and V.S. Fadin, Sov. Jour. Nucl. Phys. 50 (1989) 712;

V.S. Fadin, R. Fiore and A. Quartarolo, Phys. Rev. D53 (1996) 2729;

V. Del Duca, Phys. Rev. D54 (1996) 989;

G. Camici and M. Ciafaloni, Phys. Lett. B386 (1996) 341.

[37] R.D. Ball and S. Forte, Phys. Lett. B359 (1995) 362.

[38] R. Kirschner and L.N. Lipatov, Nucl. Phys. B213 (1983) 122.

[39] B.I. Ermolaev, S.I. Manaenkov and M.G. Ryskin, Zeit. Phys. C69 (1996) 259 ;

J. Blümlein and A. Vogt, Acta Phys. Polon. B27 (1996) 1309; Phys. Lett. B370 (1996) 149.

[40] J. Bartels, B.I. Ermolaev and M.G. Ryskin, hep-ph/9603204, preprint DESY-96-025, March 1996; Zeit. Phys. C70 (1996) 273;

J. Blümlein and A. Vogt, Phys. Lett. B386 (1996) 350; hep-ph/9610203, Proc. of the 1996 HERA Physics Workshop, eds. G. Ingelman, R. Klanner, and A. De Roeck, DESY, Hamburg, 1996, Vol. 2, p. 799; Proc. of the International Conference SPIN'96, Amsterdam, September 1996, eds. K. de Jager, P. Mulders et al, (World Scientific, Singapore, 1996), in print.

[41] J.C. Collins and W.K. Tung, Nucl. Phys. B278 (1986) 934.

[42] EMC collaboration: J.J. Aubert et al, Nucl. Phys. B213 (1983) 31.

[43] H1 collaboration: C. Adloff et. al., Zeit. Phys. C72 (1996) 593.

[44] CCFR collaboration: A.O. Bazarko et al, Zeit. Phys. C65 (1995) 189.

[45] WA70 collaboration: M. Bonesini et al, Zeit. Phys. C38 (1988) 371.

[46] BCDMS collaboration: A.C. Benvenuti et al, Phys. Lett. B223 (1989) 485.

[47] NMC collaboration, hep-ph/9610231, Nucl. Phys. B, in print.

[48] NMC collaboration: M. Arneodo et al, Phys. Lett. B364 (1995) 107.

[49] CCFR collaboration: P.Z. Quintas et al, Phys. Rev. Lett. 71 (1993) 1307. 
[50] E665 collaboration: M.R. Adams et al, Phys. Rev. D54 (1996) 3006.

[51] L.W. Whitlow et al, Phys. Lett. B282 (1992) 475;

L.W. Whitlow et al, preprint SLAC-357 (1990).

[52] H1 collaboration, Phys. Lett. B393 (1997) 452.

[53] M. Glück, E. Reya and M. Stratmann, Nucl. Phys. B422 (1994) 37.

[54] R.D. Ball and A. De Roeck, hep-ph/9609309, Proc. of the International Workshop on Deep Inelastic Scattering, Rome, April, 1996, in print.

[55] H1 collaboration, paper pa02-073, submitted to ICHEP 1996, (Warsaw, July, 1996).

[56] H1 collaboration, paper pa03-049, submitted to ICHEP 1996, (Warsaw, July, 1996). 


\section{Figure Captions}

Fig. 1. The description of the EMC and preliminary H1 data for $\mathcal{F}_{2}^{c}\left(x, Q^{2}\right)$ using the LORSC fit.

Fig. 2. The curves correspond to the value of the proton structure function $\mathcal{F}_{2}\left(x, Q^{2}\right)$ obtained from the leading-order, renormalization-scheme-consistent (LORSC) calculation at 12 values of $x$ appropriate for the most recent HERA data. For clarity of display we add $0.5(12-i)$ to the value of $\mathcal{F}_{2}\left(x, Q^{2}\right)$ each time the value of $x$ is decreased, where $i=1 \rightarrow 12$. The data are assigned to the $x$ value which is closest to the experimental $x$ bin (for more details see the similar figure displaying the two-loop fits in [26]). E665 data are also shown on the curves with the five largest $x$ values. The H1 and ZEUS data are normalized by 1.00 and 1.015 respectively in order to produce the best fit.

Fig. 3. The description of the EMC and preliminary $\mathrm{H} 1$ data for $\mathcal{F}_{2}^{c}\left(x, Q^{2}\right)$ using the $\mathrm{NLO}_{1}$ fit.

Fig. 4. Comparison of predictions for $\mathcal{F}_{L}\left(x, Q^{2}\right)$ using the full leading-order, renormalizationscheme-consistent (LORSC) fit and the $\mathrm{NLO}_{1}$ fit. For both sets of curves $\mathcal{F}_{L}\left(x, Q^{2}\right)$ increases with increasing $Q^{2}$ at the lowest $x$ values. 


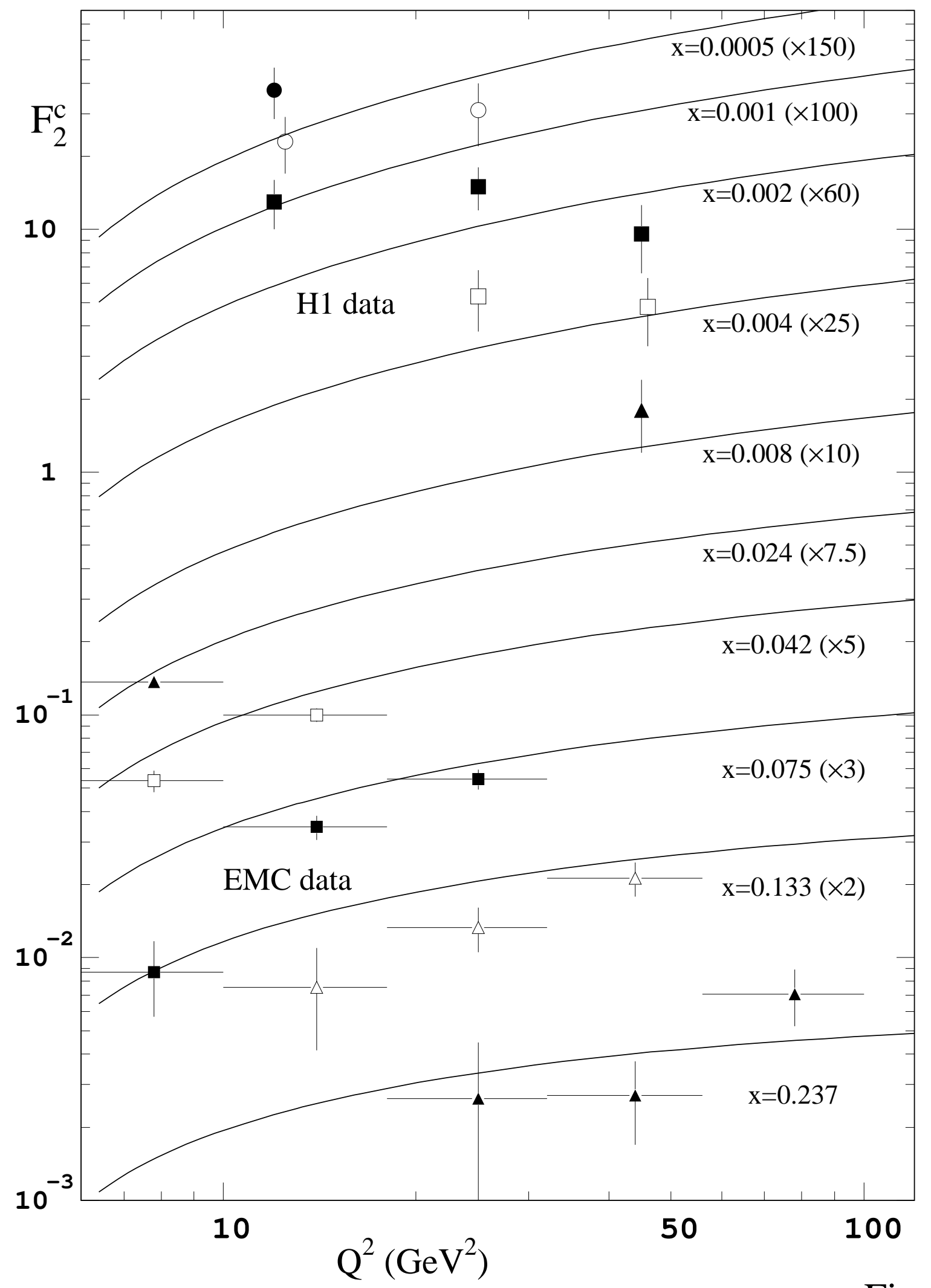

Fig. 1 


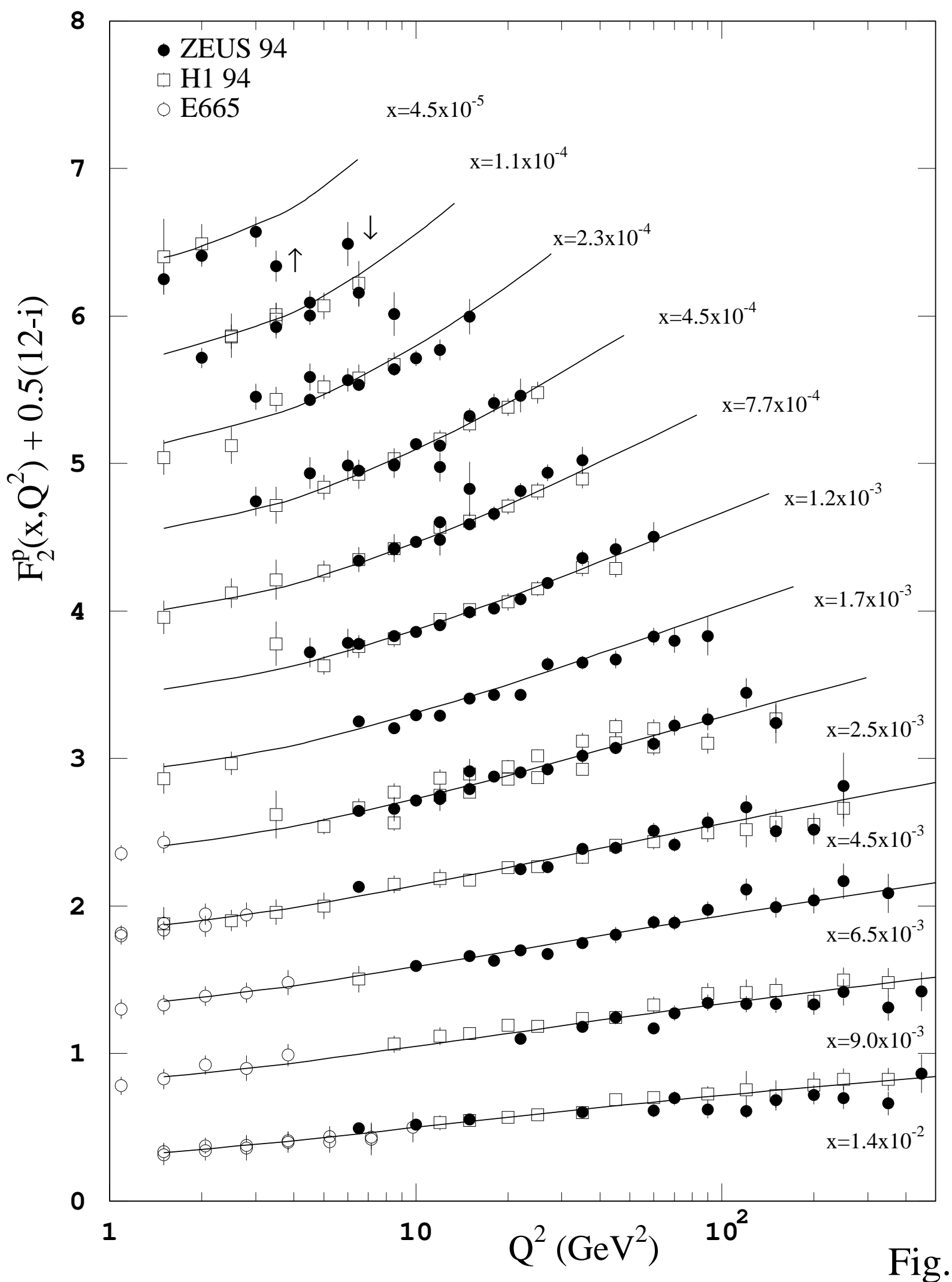

Fig. 2 


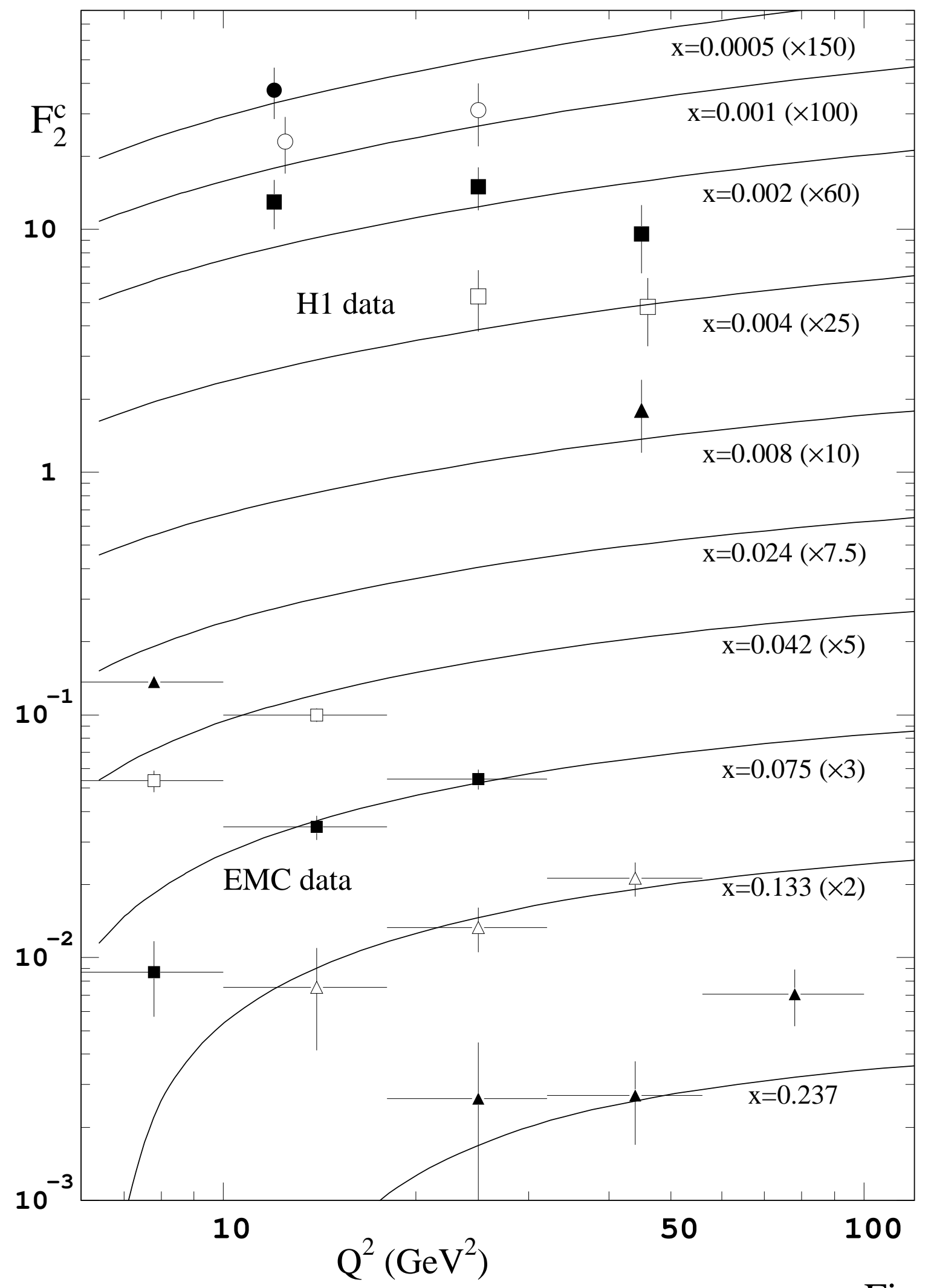

Fig. 3 


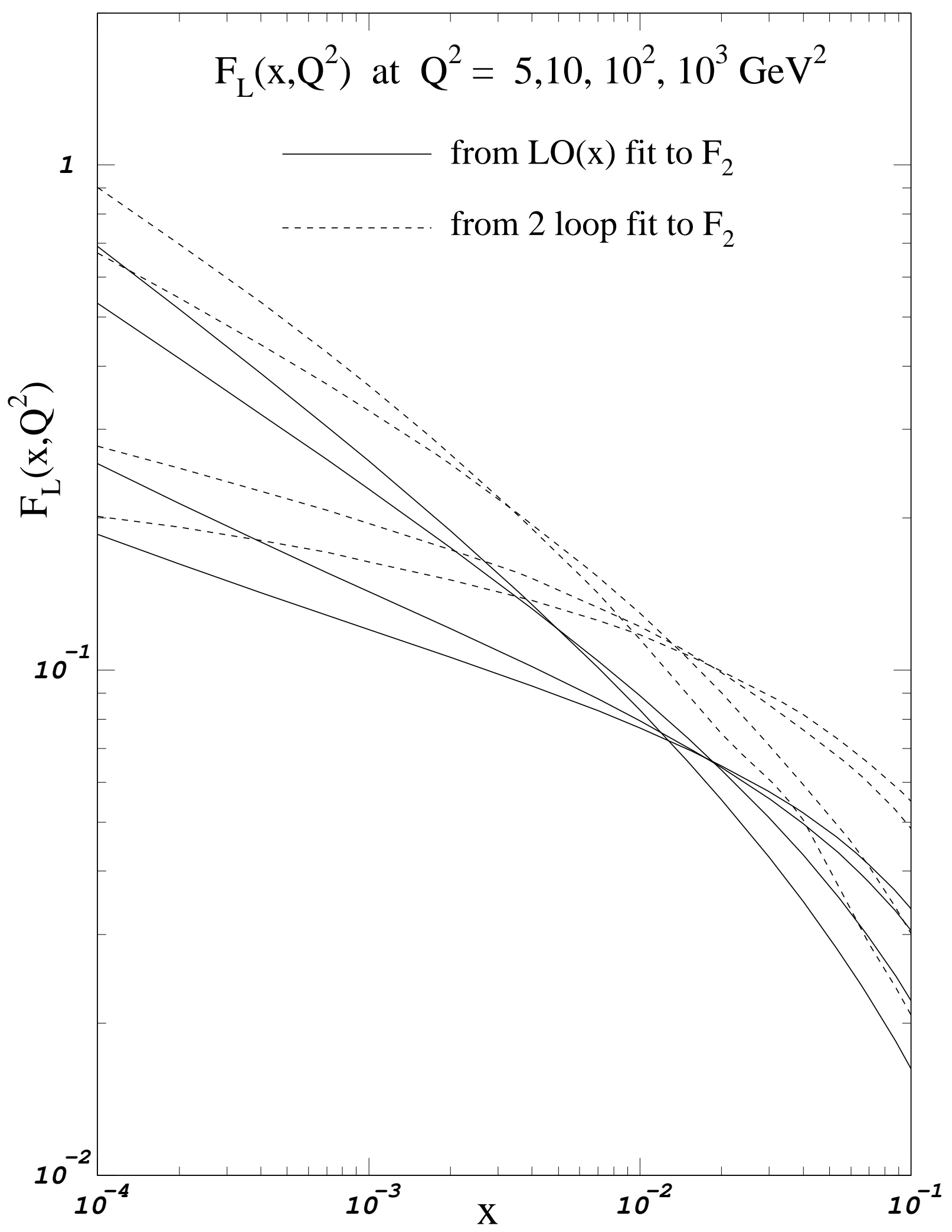

Fig. 4 